\title{
ISING INTERFACES AND FREE BOUNDARY CONDITIONS
}

\author{
CLÉMENT HONGLER AND KALLE KYTÖLÄ
}

\begin{abstract}
We study the interfaces arising in the two-dimensional Ising model at critical temperature, without magnetic field. We show that in the presence of free boundary conditions between plus and minus spins, the scaling limit of these interfaces can be described by a variant of SLE, called dipolar SLE(3). This generalizes a celebrated result of Chelkak and Smirnov [ChSm09, ChSm11] and proves a conjecture of Bauer, Bernard and Houdayer BBH05. We mention two possible applications of our result.
\end{abstract}

\section{Contents}

1. Ising model and conformal invariance 2

Acknowledgements 4

2. Main result

2.1. Statement of the main theorem

2.2. Graph and domain 6

2.3. Ising model 6

2.4. Interface 7

2.5. Dipolar SLE and Loewner chains in the strip 7

2.6. Convergence and uniformity 8

2.7. Interesting features of the proof 9

2.8. Structure of the paper 10

3. Possible Applications 10

3.1. Crossing probabilities and free boundary conditions 10

3.2. Conformal loop ensembles 11

4. Proof of the main result 14

4.1. Precompactness 14

4.2. Martingale observable 14

4.3. Identification of the scaling limit 15

5. Proof of the key theorem: the martingale observable 16

5.1. The discrete martingale observable 16

5.2. Four ingredients 18

5.3. Convergence of the discrete martingale observable 20

6. The discrete martingale property 21

6.1. Low-temperature expansion 21

6.2. Proof of Proposition 9 22

7. Kramers-Wannier duality and spin correlations 24

8. FK representation and connection events 26

8.1. The FK model 26

8.2. Correlation functions as connection probabilities 27

8.3. Discrete vertex domains 27 
8.4. Interfaces, screening effects and random domains 28

8.5. Proof of Proposition 12

8.6. Law of the conditioned FK interface 32

9. Scaling limit of elementary correlations 32

9.1. Two-point function 33

9.2. One-point function 34

9.3. Rough boundaries and uniformity 34

10. SLE variants and scaling limit of FK interfaces 35

10.1. Chordal $\operatorname{SLE}(\kappa)$ and $\operatorname{SLE}(\kappa ; \rho)$

10.2. Convergence of the FK interfaces 37

11. From FK expectations to SLE expectations 38

11.1. Proof of convergence of $\mathrm{E}_{\delta}^{\mathrm{A}}$

11.2. Proof of convergence of $\mathrm{E}_{\delta}^{\mathrm{B}} \quad 40$

12. Computation of SLE expectations 41

12.1. Computation of $\mathrm{E}^{\mathrm{A}} 43$

12.2. Computation of $\mathrm{E}^{\mathrm{B}}$

13. Discrete Complex Analysis 48

13.1. Graphs, notation and definitions 49

13.2. Discrete holomorphic observables 50

13.3. Discrete correlation functions 55

13.4. Convolution representation of discrete Riemann BVP

13.5. Continuous holomorphic observables 56

13.6. CFT correlation functions 57

13.7. Convolution representation of continuous Riemann BVP 58

13.8. Convergence of observables 60

13.9. Proof of Theorem 29 64

13.10. A priori estimates for holomorphic observables 68

Appendix A: Assumption on the vertical part of the boundary 72

Appendix B: $\operatorname{SLE}(\kappa ; \rho)$ lemmas 73

Appendix C: Control of the end of the conditioned interface 76

References

\section{Ising model and conformal invariance}

The Ising model is one of the most investigated models for order-disorder phase transitions: having a simple formulation, it exhibits a rich and interesting behavior. In two dimensions, the model can be understood at a high level of precision from both mathematical and physical viewpoints, using a variety of techniques.

Recall that the Ising model on a graph $\mathcal{G}$ is defined by a Gibbs probability measure on configurations of \pm 1 (or up/down) spins located on the vertices of $\mathcal{G}$ : it is a random assignment $\left(\sigma_{x}\right)_{x \in \mathcal{V}}$ of \pm 1 spins to the vertices $\mathcal{V}$ of $\mathcal{G}$ and the probability of a state is proportional to its Boltzmann weight $e^{-\beta \mathbf{H}}$, where $\beta>0$ is the inverse temperature of the model and $\mathbf{H}$ is the Hamiltonian, or energy, of the state $\sigma$. In the Ising model with no external magnetic field, we have $\mathbf{H}:=-\sum_{i \sim j} \sigma_{i} \sigma_{j}$, where the sum is over all the pairs of adjacent vertices of $\mathcal{G}$. 
The model favors lower energy configurations, and hence local alignment of spins: if two adjacent spins are aligned, their contribution to the energy is smaller than if they are different. The strength of this alignment effect is modulated by $\beta$. The central question is whether this local interaction gives rise to a long-range order as we take a large graph, or whether its effects remain confined to a small scale. This question turns out to depend crucially on $\beta$ : in dimension greater or equal to 2 , there exists $\beta_{c}>0$ such that for $\beta<\beta_{c}$, the system is basically disordered (except on small scales), while for $\beta>\beta_{c}$, a long-range ferromagnetic order arises (spins retain a positive correlation at arbitrarily large distance).

The Ising model was introduced by Lenz in 1920 [Len20] and its one-dimensional version was studied by Ising [Isi25]. In 1936, Peierls showed the existence of a phase transition in the Ising model in dimension two and higher by looking at the interfaces between clusters (connected components) of up and down spins [Pei36]. In 1941, Kramers and Wannier determined the value of the critical temperature on $\mathbb{Z}^{2}$, thanks to a remarkable duality result, which also deals with interfaces and is now named after them KrWa41. In 1944, Onsager computed exactly the free energy of the model at arbitrary temperatures, thus allowing for a derivation of the thermodynamic properties of the model Ons44. Since then, the two-dimensional Ising model has attracted a lot of attention, and great progress has been made, making it possible to understand the model at a rather unique level of precision [Bax89, McWu73, Pal07].

Arguably, the most intriguing and physically relevant phase of the Ising model is the critical phase and its vicinity. The advent of the Renormalization Group in the 1960s (see [Fis98] for a historical exposition) yielded a deep physical understanding (though non-rigorous) of this regime and suggested the existence of a scaling limit of the model, a universal object with continuous symmetries.

The idea that the critical scaling limits in two dimensions are conformally invariant, together with the introduction of an operator algebra for the Ising model KaCe71], suggested the description of the Ising model by Conformal Field Theory (CFT), a theory initiated by Belavin, Polyakov and Zamolodchikov BPZ84a, BPZ84b]: there should be a quantum field theory underlying the critical scaling limit, invariant by conformal transformations.

One of the most spectacular results of CFT is the prediction of exact formulae for the correlation functions of various models, in particular the Ising model. The development of boundary CFT, initiated by Cardy [Car84, subsequentially allowed to understand in a precise way the effect of the geometry of the surface on which the model lives, and the effect of various boundary conditions. One of the most emblematic successes of boundary CFT was Cardy's crossing probability formula for percolation in a conformal rectangle Car92, whose numerical verification gave one of the most convincing evidence of the full conformal invariance of that model, that is, the conformal invariance by the infinite-dimensional family of the conformal mappings.

The introduction of Schramm's SLE curves Sch00 in 1999 was the starting point of the development of the mathematical subject of conformal invariant processes. A precise sense of conformal invariance of statistical mechanics models was given, in terms of the (scaling limit of the) curves arising in the models. Shortly thereafter, the conformal invariance of the scaling limit of critical percolation on the triangular 
lattice was proven by Smirnov [Smi01], and similar results were derived for a number of other models Ken00, LSW04, Mil10, ScSh09. More recently, major progress has been realized for the Ising model and its random-cluster representation (also known as FK representation), where the interfaces arising with so-called Dobrushin boundary conditions at criticality have been shown to be conformally invariant in the scaling limit by Chelkak and Smirnov [Smi06, Smi10a, Smi10b, ChSm09, ChSm11].

While being definite breakthroughs, these results do not answer directly all questions about the conformal invariance of the Ising model. They show conformal invariance of scaling limits of the interfaces arising in a particular setup. From these results, much information can be inferred, and other scaling limit results for other types of interfaces can be obtained: for instance the convergence of all the interfaces arising with certain boundary conditions can then be expected and in principle proved, as was done for percolation [CaNe07b, Smi09]. However, proving such results is in general highly non-trivial. Moreover, there is one type of boundary conditions, conjectured to be conformally invariant, which is not directly tractable from the existing results: the free boundary conditions, which do not appear in the setup of the result of Chelkak and Smirnov.

In this paper, we generalize the result of Chelkak and Smirnov to the case when free boundary conditions enter the picture. To prove our result, we relate it to the rigorous computation of a (dual) boundary CFT correlation function, which is obtained by using both recent results concerning the boundary correlation functions of the model and existing SLE results (for dual models). Our result relies mostly on the following recent results:

- The convergence of critical FK-Ising interfaces to SLE(16/3) [Smi06].

- The scaling limits of Ising and FK-Ising fermionic observables ChSm09, Hon10a, HoSm10b.

- The precompactness and Löwner regularity of interfaces satisfying crossing estimates [KeSm11a].

- Crossing estimates for critical Ising and FK-Ising models ChSm09, DHN11.

A first promising application of our theorem is the conformal invariance of crossing probabilities investigated by Langlands, Lewis and Saint-Aubin [LLS00]: we can represent the crossing events that they consider in terms of an exploration process, whose conformally invariant scaling limit can be identified using our result. A second potential application is the proof that the collection of the Ising model interfaces converges to the Conformal Loop Ensemble (CLE) introduced by Sheffield [She09]. This also suggests the introduction of a new object to describe the collection of interfaces with free boundary conditions.

\section{Acknowledgements}

The authors would like to thank Dmitry Chelkak and Stanislav Smirnov for many enlightening conversations and useful advice, as well as Konstantin Izyurov, Wendelin Werner, Hugo Duminil-Copin, Vincent Beffara, Yvan Velenik, Pierre Nolin, Antti Kemppainen, Scott Sheffield, Julien Dubédat and Fredrik Johansson Viklund for interesting discussions. 
This research was partially supported by the Swiss NSF, the European Research Council AG CONFRA, the Academy of Finland and by the National Science Foundation under grant DMS-1106588.

\section{Main result}

2.1. Statement of the main theorem. The most natural setup to study the Ising model interfaces consists in the Dobrushin boundary conditions: take a suitable discretization of a simply connected domain, split the boundary into two connected pieces, and consider the Ising model at critical temperature on this discretization, conditioning the spins on one piece to be +1 and the ones on the other piece to be -1 . An interface naturally arises between the + and - spin clusters of the two pieces of the boundary (see Figure 2.1); for a more precise definition, see Sections $2.2,2.32 .4$ below.

The conformal invariance of the scaling limit of the interfaces appearing in the critical Ising model on the square lattice (as well as on more general graphs) with these boundary conditions was recently shown by Chelkak and Smirnov. At subcritical temperature $\left(\beta>\beta_{c}\right)$, these interfaces were shown by Pfister and Velenik to converge to a straight line [PfVe99].

Our result is the proof of a conjecture of Bauer, Bernard and Houdayer [BBH05]. The result deals with what appears to be the most natural setup involving free boundary conditions, expected to be the third type (in addition to + and - ) of conformally invariant boundary conditions [DMS97].

Theorem 1. Let $\left(D_{\delta}, r_{\delta}, \ell_{\delta}, b_{\delta}\right)_{\delta>0}$ be a family of (simply connected) discrete square grid domains of mesh size $\delta$ with three boundary marked points approximating a continuous domain $(D, r, \ell, b)$ as $\delta \rightarrow 0$.

Consider the Ising model at critical temperature on the faces of $\left(D_{\delta}, r_{\delta}, \ell_{\delta}, b_{\delta}\right)$ with free boundary condition on the counterclockwise arc $\left[r_{\delta}, \ell_{\delta}\right]$, - boundary condition on $\left[\ell_{\delta}, b_{\delta}\right]$ and + boundary condition on $\left[b_{\delta}, r_{\delta}\right]$ (see Figure 2.1).

Then, as $\delta \rightarrow 0$, the law of the initial segments of the interface $\gamma_{\delta}$ emanating at $b_{\delta}$, that separates the - spin cluster of $\left[\ell_{\delta}, b_{\delta}\right]$ and the + spin cluster of $\left[b_{\delta}, r_{\delta}\right]$ and ends on $\left[r_{\delta}, \ell_{\delta}\right]$, converges to the law of dipolar $S L E(3)$ in $(D, r, \ell, b)$.

The convergence is locally uniform with respect to the domains.

The discrete domains are defined in Section 2.2, the Ising model with boundary conditions in Section 2.3, the interface $\gamma_{\delta}$ in Section 2.4, dipolar SLE in Section 2.5. The notions of convergence and uniformity involved are briefly discussed in Section 2.6 .

Remark 2. With the recently announced results in [Che11, CDH11, one can consider the scaling limit of the whole discrete interface $\gamma_{\delta}$ and not just of its initial segments (see Section 4.1).

Remark 3. In this article we only consider square grid domains for simplicity, although our result can be generalized to other lattices as well, using techniques introduced in ChSm11, ChSm09. 

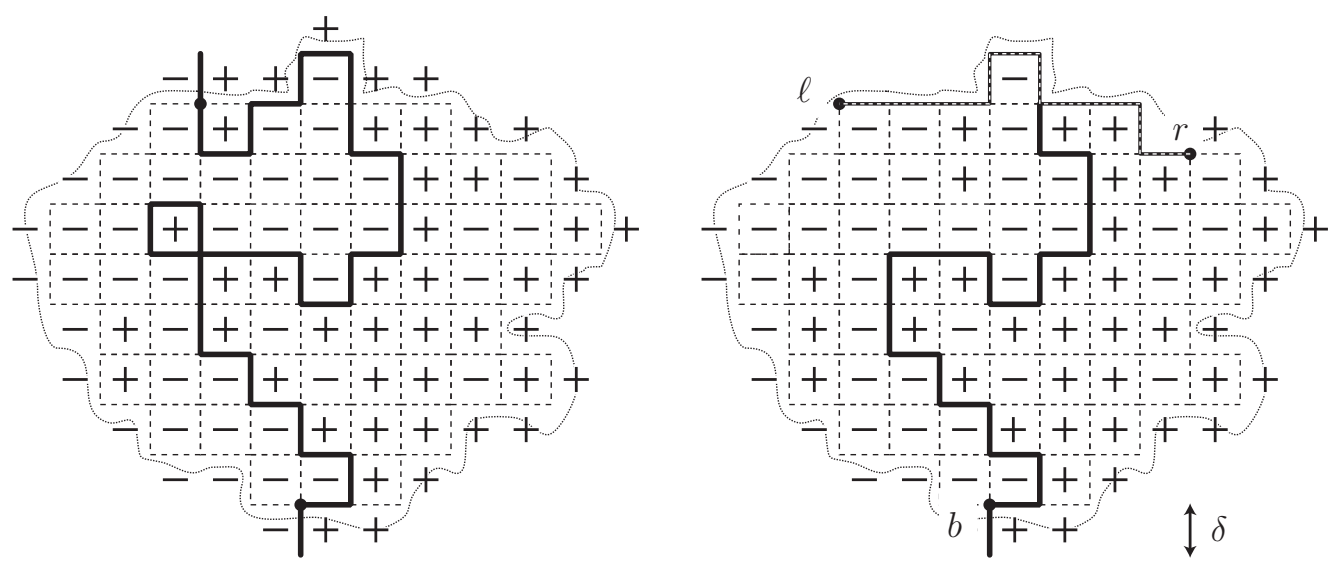

FiguRE 2.1. On the left: Ising interface with Dobrushin boundary conditions. On the right: Ising interface with free boundary condition on $[r, \ell],-$ boundary condition on $[\ell, b]$ and + boundary condition on $[b, r]$.

2.2. Graph and domain. Let us now give the notation that will be used throughout this paper:

- For $\delta>0$, we denote by $\mathbb{C}_{\delta}:=\delta \mathbb{Z}^{2}$ the square grid of mesh size $\delta$.

- A discrete square grid domain $\Omega_{\delta}$ is a simply connected graph made of the union of faces of $\mathbb{C}_{\delta}$; its boundary $\partial \Omega_{\delta}$ is a simple closed curve made of edges of $\mathbb{C}_{\delta}$; when necessary we will identify $\Omega_{\delta}$ with the Jordan domain of $\mathbb{C}$ bounded by $\partial \Omega_{\delta}$.

- For any two vertices $x, y \in \partial \Omega_{\delta}$, we denote by $[x, y] \subset \partial \Omega_{\delta}$ the counterclockwise arc between $x$ and $y$.

- When needed we will identify each edge of $\partial \Omega_{\delta}$ with the face of $\mathbb{C}_{\delta} \backslash \Omega_{\delta}$ that is adjacent to it.

- We denote by $\left(\Omega_{\delta}, a_{\delta}^{1}, \ldots, a_{\delta}^{k}\right)$ a discrete domain $\Omega_{\delta}$ with $k$ marked vertices $a_{\delta}^{1}, \ldots, a_{\delta}^{k} \in \partial \Omega_{\delta}$ appearing in counterclockwise order.

- We call an arc $\left[x_{\delta}, y_{\delta}\right] \subset \partial \Omega_{\delta}$ whose edges are all vertical a vertical arc.

We will omit a number of $\delta$ subscripts when they will be clear from the context, in particular when we will be discussing purely discrete statements.

2.3. Ising model. For concreteness and simplicity we only define here the Ising model in the setup needed for our result, that is, the critical Ising model on the faces $\mathcal{F}$ of a discrete domain $D_{\delta}$ with free boundary condition on $[r, \ell]$, - boundary condition on $[\ell, b]$ and + boundary condition on $[b, r]$. We call these boundary conditions dipolar boundary conditions on $\left(D_{\delta}, r, \ell, b\right)$. The probability space is

$$
\mathcal{S}:=\left\{\left(\sigma_{f}\right)_{f \in \mathcal{F}}: \sigma_{f} \in\{ \pm 1\} \forall f \in \mathcal{F}\right\} .
$$

The probability of a spin configuration $\sigma \in \mathcal{S}$ is given by $\mathbb{P}\{\sigma\}:=\frac{1}{\mathcal{Z}} e^{-\beta \mathbf{H}(\sigma)}$, where

- the inverse temperature $\beta$ is equal to its critical value $\frac{1}{2} \ln (\sqrt{2}+1)$; 
- the energy $\mathbf{H}(\sigma)$ is defined by

$$
\mathbf{H}(\sigma):=-\left(\sum_{f \sim g} \sigma_{f} \sigma_{g}+\sum_{f \sim[b, r]} \sigma_{f}-\sum_{f \sim[\ell, b]} \sigma_{f}\right),
$$

where the first sum is over all pairs of adjacent faces in $\mathcal{F}$, the second and third ones are respectively over all faces adjacent to an edge of $[b, r]$ and $[\ell, b]$ (a face appears several times in the sum if it is adjacent to several such edges);

- the partition function $\mathcal{Z}$ is defined as $\sum_{\sigma \in \mathcal{S}} \exp (-\beta \mathbf{H}(\sigma))$.

Notice that the boundary conditions only appear in the Hamiltonian. Another way of formulating the boundary conditions is to say that there is a +1 spin at the faces identified with $[b, r]$, that there is a -1 spin on those identified with $[\ell, b]$ and that there are no spins on the faces identified with $[r, \ell]$.

2.4. Interface. The boundary conditions of the Ising model in $\left(D_{\delta}, r, \ell, b\right)$ defined in the previous subsection (dipolar boundary conditions) naturally generate an interface between the - cluster of the arc $[\ell, b]$ and the + cluster of the arc $[b, r]$. For any configuration $\sigma \in \mathcal{S}$ (where $\mathcal{S}$ is as in Section 2.3), we can find a path $\gamma_{\delta}$ made of edges of $D_{\delta}$, that starts at $b$ and ends on $[r, \ell]$, and such that $\gamma_{\delta}$ has only faces with - spins on its left (possibly including the faces identified with $[\ell, b]$ ) and faces with + spins on its right (possibly including those identified with $[b, r]$ ), as shown on Figure 2.1. We call such a path an admissible interface.

As the square grid is not a trivalent graph, there might be different admissible choices of the interfaces, yielding ambiguities in the definition of the interface $\gamma_{\delta}$. These ambiguities turn out to be irrelevant in the scaling limit, but for definiteness, we will make the following convention.

Definition. We define the interface $\gamma_{\delta}$ to be the left-most admissible interface.

Exactly the same arguments as the ones we use in this paper give that the rightmost admissible interface converges to the same limit as the left-most one, and hence all admissible choices also converge to the same limit.

For technical reasons, we will consider initial segments of the interface, that is, the interface stopped as it hits an $\epsilon$-neighborhood of $[r, \ell]$, for an $\epsilon>0$ fixed. The scaling limit of the initial segments hence means: we let the mesh size $\delta \rightarrow 0$ with $\epsilon>0$ fixed and after that let $\epsilon \rightarrow 0$.

2.5. Dipolar SLE and Loewner chains in the strip. Schramm-Loewner Evolutions [Sch00] are the natural candidates for the conformally invariant scaling limits of discrete curves in two dimensions, as shown by Schramm's principle (see also [Kem10] for an extension of this principle relevant for our setup). See [Law05] for a reference about SLE processes.

We now define the variant of SLE suited for our purposes, which is called dipolar $S L E(\kappa)$ (see [BBH05]). It can be viewed as a particular case of the more general $\operatorname{SLE}(\kappa ; \rho)$ processes [Wer04, ScWi05, which will be introduced in Section 10.

Dipolar $\operatorname{SLE}(\kappa)$ has been shown to be the scaling limit of the loop-erased random walk from a point to an arc (when $\kappa=2$ ) Zha04 and of discrete Gaussian free field level lines with certain symmetric boundary conditions (when $\kappa=4$ ) [ScSh09. 
2.5.1. Dipolar $\operatorname{SLE}(\kappa)$. For $\kappa \geq 0$, dipolar $\operatorname{SLE}(\kappa)$ is naturally defined on the strip $\mathbb{S}:=\{z \in \mathbb{C}: 0<\Im \mathfrak{m}(z)<\pi\}$, as a Loewner chain (see Figure 2.2).

A Loewner chain in the strip is defined by the following flow equation

$$
\begin{aligned}
\partial_{t} g_{t}(z) & =\operatorname{coth}\left(\frac{g_{t}(z)-U_{t}}{2}\right) \\
g_{0}(z) & =z
\end{aligned}
$$

where $\left(U_{t}\right)_{t \geq 0}$ is a continuous real-valued function, called the driving function. Consider the Loewner chain obtained by taking as driving function $\left(\sqrt{\kappa} B_{t}\right)_{t>0}$, where $B_{t}$ is a standard one-dimensional Brownian motion. We call this chain the dipolar $S L E(\kappa)$ Loewner chain. For each $t \geq 0$, let $S_{t} \subset \mathbb{S}$ be the set of points for which the flow is well-defined up to time $t$. The following properties are valid at all times $t \geq 0$ :

- $g_{t}: S_{t} \rightarrow \mathbb{S}$ is a conformal mapping, with $\lim _{z \rightarrow+\infty} g_{t}(z)-z-t=0$ and $\lim _{z \rightarrow-\infty} g_{t}(z)-z+t=0$.

- $S_{t}$ is the unbounded connected component of $\mathbb{S} \backslash \gamma[0, t]$, where $\gamma \subset \overline{\mathbb{S}}$ is a curve, called the trace, which is such that $g_{t}(\gamma(t))=U_{t}$.

- $\gamma(0)=0$ and $\gamma(t)$ tends to a point on the upper side of $\mathbb{S}$ as $t \rightarrow \infty$.

Dipolar $\operatorname{SLE}(\kappa)$ in the strip $\mathbb{S}$ is the trace $\gamma$, considered as an (oriented) unparametrized curve.

In a domain $(D, r, \ell, b)$, dipolar $\operatorname{SLE}(\kappa)$ is defined as the image of dipolar $\operatorname{SLE}(\kappa)$ by the conformal mapping $\varphi: \mathbb{S} \rightarrow D$, with $\varphi(0)=b, \varphi(\infty)=r, \varphi(-\infty)=\ell$. In the case we are interested in (i.e. $\kappa=3$ ), dipolar $\operatorname{SLE}(\kappa)$ is almost surely a simple curve - this is true for all $\kappa \in[0,4]$ (see [Law05] for a proof in the case of chordal $\operatorname{SLE}(\kappa)$ - chordal and strip $\operatorname{SLE}(\kappa)$ are absolutely continuous with respect to each other[ScWi05]).

2.5.2. Loewner chain in the strip. As explained above, given a real-valued continuous function $\left(U_{t}\right)_{t \geq 0}$, we can generate a Loewner chain in $\mathbb{S}$ and hence a family of shrinking subdomains $\left(S_{t}\right)_{t \geq 0}$ of $\mathbb{S}$, with $S_{t} \subset S_{s}$ for any $t \geq s$ and $S_{0}=\mathbb{S}$. Conversely, it can be shown (see [Law05]) that any such family of subdomains $\left(S_{t}\right)_{t \geq 0}$ satisfying a certain local growth property can be realized (after time reparametrization) as a Loewner chain in the strip, guided by a continuous driving function $\left(V_{t}\right)_{t \geq 0}$.

2.6. Convergence and uniformity. As for most SLE convergence results, there are actually several types of convergence results that can be obtained with our techniques: the strength of the result we get depends on how well (in which topology) the discrete domains $\left(D_{\delta}, r_{\delta}, \ell_{\delta}, b_{\delta}\right)$ approximate the continuous domain $(D, r, \ell, b)$. For definiteness and simplicity, we will use a rather strong topology, which is best suited for applications.

For two oriented simple curves $\gamma_{1}, \gamma_{2}$ in the complex plane, we define $\mathrm{d}_{\infty}\left(\gamma_{1}, \gamma_{2}\right)$ by

$$
\mathrm{d}_{\infty}\left(\gamma_{1}, \gamma_{2}\right):=\inf _{\zeta_{1}, \zeta_{2}}\left\|\zeta_{1}-\zeta_{2}\right\|_{\infty}
$$

where the infimum is taken over all orientation-preserving parametrizations $\zeta_{1}$ and $\zeta_{2}$ of $\gamma_{1}$ and $\gamma_{2}$ respectively. Let $\mathrm{C}$ be the completion of the set of simple curves for 


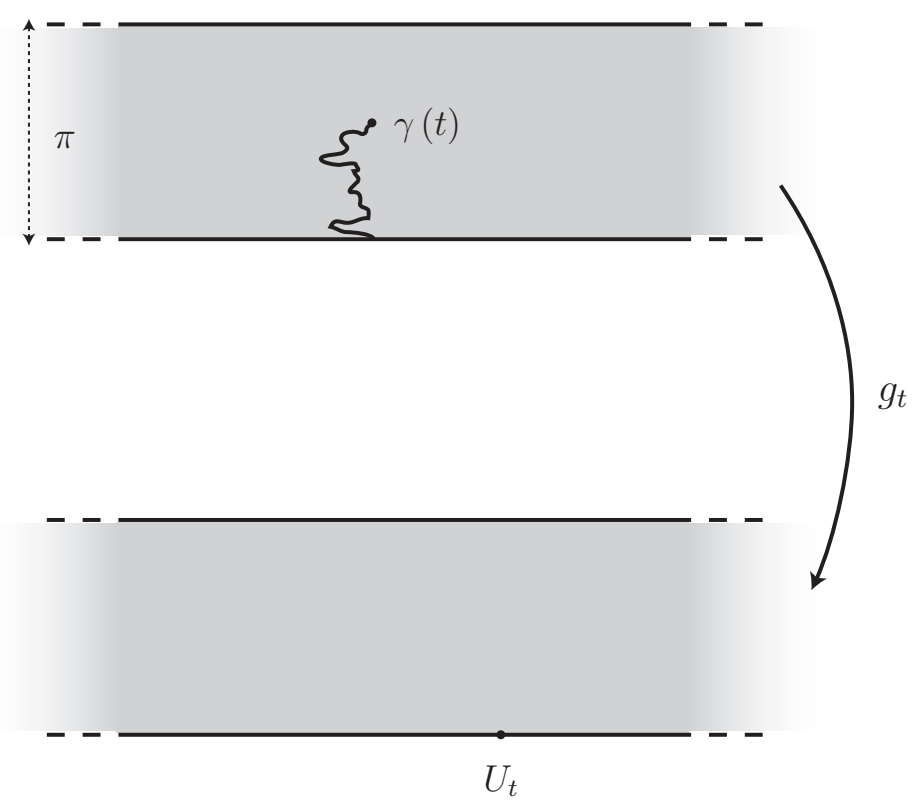

FiguRE 2.2. Loewner chain in the strip

this metric. For two domains $\left(D_{1}, a_{1}^{1}, \ldots, a_{n}^{1}\right)$ and $\left(D_{2}, a_{2}^{1}, \ldots, a_{n}^{2}\right)$ with $n$ marked boundary points such that $\partial D_{1}, \partial D_{2} \in \mathrm{C}$, we define

$$
\mathrm{d}_{\infty}\left[\left(D_{1}, a_{1}^{1}, \ldots, a_{n}^{1}\right),\left(D_{2}, a_{1}^{1}, \ldots, a_{n}^{2}\right)\right]=\mathrm{d}_{\infty}\left(\partial D_{1}, \partial D_{2}\right)+\sum_{i=1}^{n}\left|a_{i}^{1}-a_{i}^{2}\right| .
$$

We can now define the type of convergence we will work with:

- We say that $\left(D_{\delta}, r_{\delta}, \ell_{\delta}, b_{\delta}\right) \rightarrow(D, r, \ell, b)$ if $\mathrm{d}_{\infty}\left[\left(D_{\delta}, r_{\delta}, \ell_{\delta}, b_{\delta}\right),(D, r, \ell, b)\right] \rightarrow 0$ as $\delta \rightarrow 0$.

- We say that the interface $\gamma_{\delta}$ converges in law to the dipolar SLE trace $\gamma$ as $\delta \rightarrow 0$ if for any $\epsilon>0$, there exists $\delta_{0}>0$ such that for any $\delta \leq \delta_{0}$, there exists a coupling of $\gamma_{\delta}$ and $\gamma$ such that $\mathbb{P}\left\{\mathrm{d}_{\infty}\left(\gamma_{\delta}, \gamma\right)>\epsilon\right\} \leq \epsilon$. This is equivalent to saying that $\gamma_{\delta}$ converges weakly to $\gamma$.

What we mean by locally uniform convergence in Theorem 1 is: for any $R>0$ and any $\epsilon>0$, there exists $\delta_{0}, \epsilon_{0}>0$ such that for any $\delta \leq \delta_{0}$, for any discrete domain $\left(D_{\delta}, r_{\delta}, \ell_{\delta}, b_{\delta}\right)$ of diameter smaller than $R$, such that

$$
\mathrm{d}_{\infty}\left[\left(D_{\delta}, r_{\delta}, \ell_{\delta}, b_{\delta}\right),(D, r, \ell, b)\right] \leq \epsilon_{0},
$$

we have that there exists a coupling of the interface $\gamma_{\delta}$ in $\left(D_{\delta}, r_{\delta}, \ell_{\delta}, b_{\delta}\right)$ and the SLE $\gamma$ in $(D, r, \ell, b)$ such that:

$$
\mathbb{P}\left\{\mathrm{d}_{\infty}\left(\gamma_{\delta}, \gamma\right)>\epsilon\right\} \leq \epsilon
$$

2.7. Interesting features of the proof. Although our proof follows a classical strategy for proving convergence results to SLE, it involves a number of ideas that are new in the subject. In particular, we find the following features worth pointing out:

- Our new martingale observable is not a discrete holomorphic or discrete harmonic function, for it does not satisfy local relations. Instead, it is merely 
defined on the boundary of the domain where we are considering it. For that reason, it requires more than discrete complex analysis to show the convergence of the observable to a conformally invariant limit.

- To understand the scaling limit of the Ising model, one introduces and studies the scaling limit of a dual Ising model.

- One uses $\operatorname{SLE}(16 / 3)$ to obtain a convergence result to SLE(3): scaling limits of correlation functions of the dual Ising model can be expressed as SLE(16/3) integrals that can then be computed using Itô's calculus.

- The proof illustrates the usefulness of obtaining exact results for quantities like the spin correlations to derive a qualitative result, the conformal symmetry of certain Ising interfaces.

- The non-universal (lattice-dependent) multiplicative constants appearing in the exact formulae for the correlation functions that we compute turn out to be useful to show the convergence to a universal limit.

- The proof demonstrates the possibility to use local Riemann charts together with discrete complex analysis to understand boundary correlation functions for the Ising model on rough domains.

2.8. Structure of the paper. In Section 3, we give two possible applications of our result, to the computation of crossing probabilities and to the convergence of the Ising interfaces to Conformal Loop Ensembles.

The rest of this paper is then devoted to the proof of Theorem 1. The global strategy is the following:

- In Section 4, Theorem 1 is reduced to a key theorem (Theorem 6), which is the existence of a so-called continuous martingale observable available in the scaling limit, following a path that has now become standard in the SLE subject.

- In Section 5, one constructs a discrete martingale observable for the interface (Proposition 9, proven in Section 6). The heart of the matter to prove the key Theorem 6 is to show that the discrete martingale observable converges to the continuous one. This convergence result is decomposed into four ingredients (Propositions 11, 12, 14 and 16), which are proven in Sections 7, 8, 9 and 11 respectively.

- The discrete complex analysis techniques required to prove the results of Section 9 are finally presented in Section 13

\section{Possible Applications}

3.1. Crossing probabilities and free boundary conditions. In [LLS00, Langlands, Lewis and Saint-Aubin investigated numerical evidence for the conformal invariance of the Ising model, taking a approach similar to the one of [LPS94] for percolation. They considered probabilities of crossings made of + spins in conformal rectangles (simply connected domains with four marked boundary points), with free boundary conditions, and concluded the conformal invariance of the scaling limit of these probabilities. More precisely, they gave numerical evidence suggesting the following: 

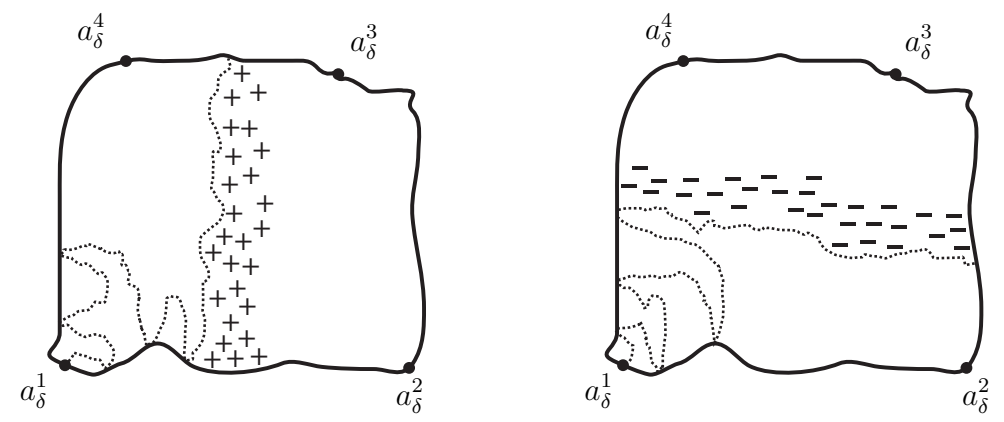

FiguRE 3.1. Depending on whether there is or there is not a crossing of + spins $\left[a_{\delta}^{1}, a_{\delta}^{2}\right] \leadsto\left[a_{\delta}^{3}, a_{\delta}^{4}\right]$, the exploration process $\iota_{\delta}$ (dotted) first hits $\left[a_{\delta}^{3}, a_{\delta}^{4}\right]$ or $\left[a_{\delta}^{2}, a_{\delta}^{3}\right]$; if there is no + crossing, there is a star-crossing (see [Wer10]) of - spins $\left[a_{\delta}^{2}, a_{\delta}^{3}\right] \leadsto\left[a_{\delta}^{4}, a_{\delta}^{1}\right]$.

Problem. Show that for any simply connected domain with four marked boundary points $\left(D, a^{1}, a^{2}, a^{3}, a^{4}\right)$, there exists a correlation function $\mathfrak{C}\left(D, a^{1}, a^{2}, a^{3}, a^{4}\right)$, which is conformally invariant in the sense that

$$
\mathfrak{C}\left(\varphi(D), \varphi\left(a^{1}\right), \varphi\left(a^{2}\right), \varphi\left(a^{3}\right), \varphi\left(a^{4}\right)\right)=\mathfrak{C}\left(D, a^{1}, a^{2}, a^{3}, a^{4}\right)
$$

for any conformal mapping $\varphi: D \rightarrow \varphi(D)$ and such that if $\left(D_{\delta}, a_{\delta}^{1}, a_{\delta}^{2}, a_{\delta}^{3}, a_{\delta}^{4}\right)$ is a family of discrete domains approximating $\left(D, a^{1}, a^{2}, a^{3}, a^{4}\right)$ and we consider the critical Ising model on $\left(D_{\delta}, a_{\delta}^{1}, a_{\delta}^{2}, a_{\delta}^{3}, a_{\delta}^{4}\right)$ with free boundary conditions, we have

$$
\mathbb{P}_{D_{\delta}}\left\{\text { there is a crossing of }+ \text { spins }\left[a_{\delta}^{1}, a_{\delta}^{2}\right] \leadsto\left[a_{\delta}^{3}, a_{\delta}^{4}\right]\right\} \underset{\delta \rightarrow 0}{\longrightarrow} \mathfrak{C}\left(D, a^{1}, a^{2}, a^{3}, a^{4}\right) .
$$

We say that there is a crossing of + spins $\left[a_{\delta}^{1}, a_{\delta}^{2}\right] \leadsto\left[a_{\delta}^{3}, a_{\delta}^{4}\right]$ when there is a connected component of $D_{\delta}$ that is adjacent to $\left[a_{\delta}^{1}, a_{\delta}^{2}\right]$ and $\left[a_{\delta}^{3}, a_{\delta}^{4}\right]$, the spins at the vertices thereof are all +1 .

In a subsequent paper, the authors and Hugo Duminil-Copin will show this conformal invariance result, whose proof relies on Theorem 1. The strategy resembles the SLE-based derivation of Cardy's formula for percolation (see [LSW01]):

- One translates the crossing events in terms of hitting probabilities for a discrete exploration path: construct an exploration process $\iota_{\delta}$ started at $a_{\delta}^{1}$ that has - spins on its left and + spins on its right, and which "pretends" that there are - spins on $\left[a_{\delta}^{4}, a_{\delta}^{1}\right]$ and that there are + spins on $\left[a_{\delta}^{1}, a_{\delta}^{2}\right]$. Depending on whether $\iota_{\delta}$ first hits $\left[a_{\delta}^{3}, a_{\delta}^{4}\right]$ or $\left[a_{\delta}^{2}, a_{\delta}^{3}\right]$, there is or there is not a crossing of + spins $\left[a_{\delta}^{1}, a_{\delta}^{2}\right] \leadsto\left[a_{\delta}^{3}, a_{\delta}^{4}\right]$ (see Figure 3.1).

- One shows that the discrete exploration path converges in law to a conformally invariant continuous process: using a priori estimates, one gets that the subsequential scaling limits of the process are instantenously reflected on $\partial D_{\delta}$, and, using the main result of the present paper, that the excursions are described by dipolar $\operatorname{SLE}(3)$.

3.2. Conformal loop ensembles. The most natural geometrical object to describe an Ising model configuration on a discrete domain $D_{\delta}$ (with + boundary conditions, say) is probably the collection of all interfaces between + and - spin clusters (in other words: put a dual edge between any pair of spins with opposite signs), which 
form a collection of nested loops on the lattice. The study of such contours dates back to Peierls, who showed the existence of a phase transition by such considerations [Pei36] (see also the contours of Lemma 17).

At critical temperature, it is natural to expect these random loops to have a scaling limit, and the limiting loops to look like a variant of $\operatorname{SLE}(3)$. This limit, called Conformal Loop Ensemble (CLE) with $\kappa$ parameter equal to 3, is indeed a random collection of continuous loops that can be constructed from SLE.

The CLE $(\kappa)$ processes, introduced in [She09], are defined for $\kappa \in(8 / 3,8]$, and they are the conjectural scaling limits of loops arising in various lattice models; for $\kappa \in(8 / 3,4]$, they also can be constructed from a Brownian loop soup [ShWe10a].

A very useful characterization result gives that the CLE $(\kappa)$ 's are the unique objects satisfying conformal invariance and an analog of the domain Markov property (that many lattice models satisfy on discrete level) [ShWe10b].

It is reasonable to expect that the convergence of all the loops of a lattice model to $\mathrm{CLE}(\kappa)$ follows from the convergence of a single interface between marked boundary point to $\operatorname{SLE}(\kappa)$. This has been worked out in detail for the case of percolation $(\kappa=$ 6) CaNe07b, and is work in progress for FK-Ising model $(\kappa=16 / 3)$ KeSm11b; there is also closely related work in progress for the uniform spanning tree $(\kappa=8)$ BeDu11.

For the Ising model, the situation seems more complicated, although it might be possible to derive the convergence to CLE(3) directly from the convergence of interfaces with $+/$ - boundary conditions to chordal SLE(3).

The core idea for both percolation and FK-Ising is to construct an exploration process on discrete level, that starts from a point on the boundary and explores all the loops of the model; what makes this idea work is that macroscopic loops touch the boundary with probability tending to 1 as the mesh size $\delta \rightarrow 0$. This way, the discrete process enters the bulk automatically and is instantaneously reflected on the boundary; its excursions can be identified using the convergence results to chordal $\operatorname{SLE}(\kappa)$.

The problem is that such an approach with chordal SLE cannot work, at least without modification, for the Ising model: indeed, with probability tending to 1 , there are no macroscopic loops touching the boundary, as is witnessed by the fact that the CLE(3) loops do not touch each other, or simply that the SLE(3) trace is a simple curve. Hence, if we use the same discrete construction as for the FKIsing model, the exploration process will get stuck on the boundary of the domain and will find no loop. It is reasonable to expect that this construction works if one introduces small jumps in the exploration process, or if we introduce some randomization procedure, but this seems rather subtle to handle

We propose here an alternative approach, which allows to explore the loops of the model with an exploration process. It relies on the two convergence results

- The convergence of the FK-Ising interfaces to CLE(16/3) (result of Kemppainen and Smirnov [KeSm11b]).

- The identification of the arcs appearing for the Ising model with free boundary conditions, using dipolar SLE(3).

Let us explain how from these two ingredients one can show the conformal invariance of the loops of the model. The Ising model with + boundary conditions can be coupled with an FK-Ising model with wired boundary conditions; through this 


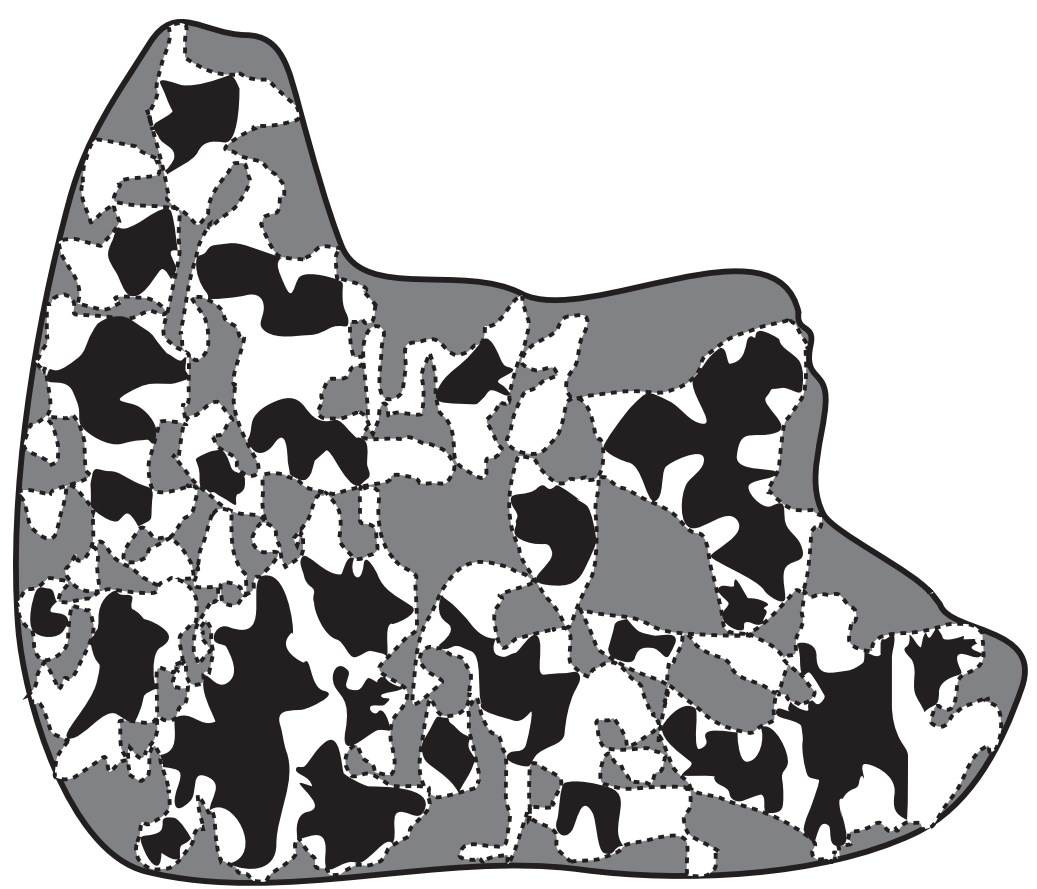

FigurE 3.2. Coupling of FK-Ising and Ising interfaces. The dashed loops, which are bounding white regions, are part of the boundary of the FK cluster $\Gamma$ (they are the $\gamma_{\delta}^{(j)}$ loops). Conditionally on these loops, the boundary conditions for the Ising model inside them are purely free. The boundaries of the black areas are the $\lambda_{\delta}^{(k ; j)}$ loops (they have + spins outside and - spins inside).

coupling, the Ising model spin configurations are obtained by assigning independent random \pm 1 values (with probabilities $1 / 2-1 / 2$ ) to the vertices of each FK cluster (see Theorem 19p. If we look at the wired cluster $\Gamma$ attached to the boundary of the domain, then the (inner) boundary of this cluster consists of disjoint loops $\gamma_{\delta}^{(1)}, \ldots, \gamma_{\delta}^{(n)}$. Conditionally on $\gamma_{\delta}^{(1)}, \ldots, \gamma_{\delta}^{(n)}$, the law of the spins (obtained through the coupling) in the domains $\Omega_{\delta}^{(1)}, \ldots, \Omega_{\delta}^{(n)}$ are the laws of independent critical Ising models with free boundary conditions (see Figure 3.2). The spins of $\Gamma$ are all set to + . Hence, we know that there are no Ising loops in $\Gamma$ : all the Ising loops (having + spins outside and - spins inside) appear inside the domains $\Omega_{\delta}^{(k)}$. Let us denote by $\lambda_{\delta}^{(k ; j)}\left(1 \leq j \leq k_{m}\right)$ the loops appearing in $\Omega_{\delta}^{(k)}$. A number of the loops $\lambda_{\delta}^{(k ; j)}$ touch the loop $\gamma_{k}$ and they can be reconstructed by pasting boundary arcs with parts of $\gamma_{k}$.

The point is that the the loops $\left\{\gamma_{\delta}^{(i)}: i\right\}$ converge to CLE(16/3) loops as $\delta \rightarrow 0$ and that the arcs on the loops $\left\{\gamma_{\delta}^{(i)}: i\right\}$ converge to a free SLE(3) tree, discussed below. Hence, we can construct the scaling limit of the loops $\lambda_{\delta}^{(k ; i)}$, which are the outermost loops of the Ising model with + boundary conditions in the original 
domain. The process can then be iterated inside the loops thus obtained, and all the loops will be eventually discovered. As the whole construction is conformally invariant, so is the scaling limit of the collection of the loops arising in the model; by the characterization of ShWe10b], we deduce that the collection of all the loops is $\operatorname{CLE}(3)$.

Let us now describe the free $\operatorname{SLE}(3)$ tree in a domain $\Omega$, which describes the scaling limit of the arcs linking the boundary with free boundary conditions. It is a continuous tree, such that any two points $x, y \in \partial \Omega$ are linked by a branch which is an exploration process as that "pretends" there is + boundary condition on $[x, y]$ and - boundary condition on $[y, x]$. For any three points $x, y, z \in \partial \Omega$, we can couple the branch $\iota^{x \rightarrow y}$ from $x$ to $y$ and the branch $\iota^{x \rightarrow z}$ from $x$ to $z$ in such a way that they are the same, up to the first time $\tau$ when $x$ and $z$ are disconnected by that branch; the remaining of the curve is independent.

Note that our technique also allows to describe the scaling limit of the collection of all the interfaces appearing with various boundary conditions, in particular purely free boundary conditions.

\section{Proof of the main result}

We now outline the proof of our main result, Theorem 1. The key argument is the martingale observable result (Theorem 6 in Section 4.2 ). Together with the precompactness result given in Section 4.1, the key theorem is used to obtain Theorem 1 in Section 4.3 .

4.1. Precompactness. The first ingredient in the proof of Theorem 1 is a precompactness result, which allows to extract subsequential limits.

Theorem 4. With the assumptions and the notation of Theorem 1, the laws of the initial segments of the interfaces $\gamma_{\delta}$ form a tight family, and their subsequential scaling limits are almost surely curves.

This result follows from standard estimates (Russo-Seymour-Welsh-type crossing bounds) and its proof is exactly the same as the one for the Dobrushin setup ChSm11. These are a priori uniform estimates for some crossing probabilities follow from [ChSm09, DHN11. The framework built in [KeSm11a gives the result. By these arguments, one shows that the law of the curves stopped upon reaching an $\epsilon$-neighborhood of $[r, \ell]$ are tight and by diagonal extraction one can let $\epsilon \rightarrow 0$.

To interchange the limits (which allows to consider the scaling limit of the discrete interfaces and not just initial segments thereof), one needs additional control on the end of the interface: one has to ensure that the discrete interface $\gamma_{\delta}$ hits the arc $[r, \ell]$ with high probability once it gets close to that arc. This can be deduced from strong RSW a priori estimates, which have been recently announced [Che11, CDH11].

4.2. Martingale observable. This subsection contains the key result for proving the main theorem: it is the part which is really specific to our setup.

Let us first define what is known as an observable in the SLE literature, and as a correlation function in the CFT literature: it is a function of a domain with marked points. This observable will play a crucial role in the proof of the main theorem. 
Definition 5. For any domain $(\Omega, r, \ell, x, z)$ such that $z$ is on a smooth part of $\partial \Omega$, we denote by $\Phi(\Omega, r, \ell, x, z) \in \mathbb{R}$ the quantity defined by

$$
\Phi(\Omega, r, \ell, x, z):=\sqrt{\frac{\sqrt{2}+1}{2 \pi}}\left|\psi^{\prime}(z)\right|^{\frac{1}{2}} \operatorname{coth}\left(\frac{\psi(z)}{2}\right)
$$

where $\psi$ is the conformal map from $\Omega$ to the strip $\mathbb{S}:=\{z \in \mathbb{C}: 0<\Im \mathfrak{m}(z)<\pi\}$ such that $\psi(x)=0, \psi(r)=+\infty, \psi(\ell)=-\infty$.

The key theorem to prove the convergence of the interface to SLE is the martingale property of the function $\Phi$. It brings to the continuous level all the information about the Ising model that we need to identify the curve.

Theorem 6. Assume that the arc $[b, r]$ contains a vertical part $\mathfrak{v}$ and that for each $\delta>0$ the discretization $\left(D_{\delta}, r_{\delta}, \ell_{\delta}, b_{\delta}\right)$ contains a vertical part $\mathfrak{v}_{\delta} \subset\left[b_{\delta}, r_{\delta}\right]$ that converges to $\mathfrak{v}$ as $\delta \rightarrow 0$. Let $\gamma$ have the law of any subsequential limit of (the initial segments of) discrete interfaces $\gamma_{\delta_{n}}$ for a sequence $\left(\delta_{n}\right)_{n>0}$ with $\delta_{n} \rightarrow 0$ as $n \rightarrow \infty$. Let $D_{t}$ be the connected component of $\Omega \backslash \gamma_{t}$ containing the arc $[r, \ell]$. Let $\tau \in[0, \infty]$ be the first time $t$ when $\gamma$ hits $\mathfrak{v} \cup[r, \ell]$.

Then for any $z \in \mathfrak{v}$, we have that

$$
\left(\Phi\left(D_{t \wedge \tau}, r, \ell, \gamma(t \wedge \tau), z\right)\right)_{t \geq 0}
$$

is a continuous local martingale.

In physical terms, the observable $\Phi$ hence plays the role of a one-parameter family of (stochastic) conservation laws, indexed by $z$. The proof of this theorem is discussed in Section 6 .

4.3. Identification of the scaling limit. The following technical lemma, shown in Appendix A, allows us to fit with the framework of Theorem 6 .

Lemma 7. To prove Theorem 1, we can assume that the domain $D$ is such that the arc $[b, r]$ contains a vertical part $\mathfrak{v}$ and that the discrete domains $D_{\delta}$ are such that the arc $\left[b_{\delta}, r_{\delta}\right]$ contains a vertical part $\mathfrak{v}_{\delta}$ converging to $\mathfrak{v}$ as $\delta \rightarrow 0$.

We can now give the proof of the main theorem:

Proof of Theorem 1. By Theorem 4, we can extract subsequential scaling limits of the (initial segments of the) discrete interfaces $\left(\gamma_{\delta}\right)_{\delta>0}$ as $\delta \rightarrow 0$. It remains to identify any subsequential scaling limit $\gamma$ as dipolar $\operatorname{SLE}(3)$ in $(D, r, \ell, b)$. Let us assume that we have a vertical part of the boundary $\mathfrak{v} \subset[b, r]$ as in Lemma 7. Thanks to the key Theorem 6, we can follow a procedure which has become standard in the SLE subject [Smi06, MaSm09] to identify the scaling limit of the interface:

- We describe the growing random curve by its complementary, and look, for each time $t \geq 0$, at the domain $D$ slitted by the curve $\gamma[0, t]$ (we pick an arbitrary parametrization of $\gamma$ ). Our interface is now described by shrinking domains $\left(D_{t}, r, \ell, \gamma(t)\right)_{t \geq 0}$, where $D_{t} \subset D$ is the connected component of $D \backslash \gamma[0, t]$ that contains $[r, \ell]$.

- For any $z \in \mathfrak{v}$, the process $\left(\Phi\left(D_{t}, r, \ell, \gamma(t), z\right)\right)_{t \geq 0}$, stopped as $\gamma(t)$ hits $\mathfrak{v} \cup[r, \ell]$, is a continuous local martingale (Theorem 6).

- We map $D$ to the strip $\mathbb{S}$ by the conformal mapping $\psi: D \rightarrow \mathbb{S}$ such that $\psi(b)=0, \psi(r)=\infty, \psi(\ell)=-\infty$. 
- We look at the process $\left(S_{t}\right)_{t>0}$, where for any $t \geq 0, S_{t}$ is the unbounded connected component of $\mathbb{S} \backslash \bar{\psi}(\gamma[0, t])$.

- As explained in Section 2.5.2, we can encode $\left(S_{t}\right)_{t>0}$ by a strip Loewner chain $\left(g_{t}: S_{t} \rightarrow \mathbb{S}\right)_{t \geq 0}$, with driving process $\left(V_{t}\right)_{t \geq 0}$ : after time reparametrization, we have

$$
\begin{aligned}
\partial_{t} g_{t}(z) & =\operatorname{coth}\left(\frac{g_{t}(z)-V_{t}}{2}\right), \\
g_{0}(z) & =z .
\end{aligned}
$$

- By conformal covariance of $\Phi$ and its explicit formula on the strip, we deduce that

$$
\left(\left|g_{t}^{\prime}(z)\right|^{\frac{1}{2}} \operatorname{coth}\left(\frac{1}{2}\left(g_{t}(z)-V_{t}\right)\right)\right)_{t \geq 0}
$$

is a continuous local martingale for any $z \in \psi(\mathfrak{v})$.

- Since $g_{t}(z)$ and $g_{t}^{\prime}(z)$ are differentiable in time (and hence of finite variation) and since $g_{t}^{\prime}(z)$ never vanishes, we deduce that $\left(V_{t}\right)_{t \geq 0}$ is a continuous semimartingale.

- Using that $\left(V_{t}\right)_{t \geq 0}$ is a continuous semi-martingale, we can apply Itô's calculus to get that

$$
\begin{aligned}
& \mathrm{d}\left(\left|g_{t}^{\prime}(z)\right|^{\frac{1}{2}} \operatorname{coth}\left(\frac{g_{t}(z)-V_{t}}{2}\right)\right) \\
= & \frac{\left|g_{t}^{\prime}(z)\right|^{\frac{1}{2}}}{4}\left(\frac{2 \mathrm{~d} V_{t}}{\sinh ^{2}\left(\frac{g_{t}(z)-V_{t}}{2}\right)}+\frac{\cosh \left(\frac{g_{t}(z)-V_{t}}{2}\right)}{\sinh ^{3}\left(\frac{g_{t}(z)-V_{t}}{2}\right)}\left(\mathrm{d}\left\langle V_{t}, V_{t}\right\rangle-3 \mathrm{~d} t\right)\right),
\end{aligned}
$$

for any $z \in \psi(\mathfrak{v})$. We obtain that $V_{t}$ is driftless and that $\mathrm{d}\left\langle V_{t}, V_{t}\right\rangle=3 \mathrm{~d} t$.

- Since we moreover have $V_{0}=0$ (as $\psi(\gamma)$ starts growing at 0$)$, it follows from Lévy's characterization theorem that $\left(V_{t}\right)_{t}$ has the law of $\left(\sqrt{3} B_{t}\right)_{t}$, where $\left(B_{t}\right)_{t}$ is a standard Brownian motion.

- This shows that $\psi(\gamma)$ has the law of dipolar $\operatorname{SLE}(3)$ in $(\mathbb{S}, \infty,-\infty, 0)$ and hence that $\gamma$ has the law of dipolar $\operatorname{SLE}(3)$ in $(D, r, \ell, b)$.

\section{Proof of the key theorem: the martingale observable}

5.1. The discrete martingale observable. In this section we give the main steps for the proof of the key theorem (Theorem 6). Let us first define a discrete version of the observable $\Phi$ introduced in Section 4.2 , which allows to make the connection with the Ising model:

Definition 8. Let $\left(\Omega_{\delta}, r, \ell, x, z\right)$ be a discrete domain with four marked boundary points. We denote by $\Phi_{\delta}\left(\Omega_{\delta}, r, \ell, x, z\right)$ the quantity defined by

$$
\Phi_{\delta}\left(\Omega_{\delta}, r, \ell, x, z\right):=\frac{\tilde{\mathcal{Z}}\left(\Omega_{\delta}, r, \ell, x, z\right)}{\mathcal{Z}\left(\Omega_{\delta}, r, \ell, x\right)},
$$

where 

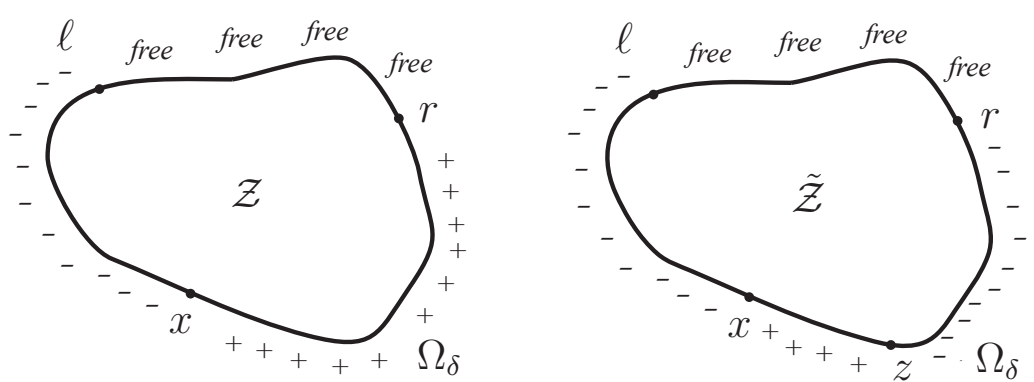

FIGURE 5.1. The boundary conditions for the partition functions $\mathcal{Z}\left(\Omega_{\delta}, r, \ell, x\right)$ and $\tilde{\mathcal{Z}}\left(\Omega_{\delta}, r, \ell, x, z\right)$.

- $\mathcal{Z}\left(\Omega_{\delta}, r, \ell, x\right)$ is the partition function of the critical Ising model on the faces of $\left(\Omega_{\delta}, r, \ell, x\right)$ with dipolar boundary conditions (free on $[r, \ell],-$ on $[\ell, x]$ and + on $[x, r])$, as defined in Section 2.3 .

- $\tilde{\mathcal{Z}}\left(\Omega_{\delta}, r, \ell, x, z\right)$ is the partition function of the critical Ising model on the faces of $\left(\Omega_{\delta}, r, \ell, x, z\right)$ with the following modified boundary conditions: free on $[r, \ell],-$ on $[\ell, x],+$ on $[x, z]$ and - on $[z, r]$; more precisely, it is obtained by replacing $[b, r]$ by $[x, z]$ and $[\ell, b]$ by $[\ell, x] \cup[z, r]$ in Equation 2.1.

The following proposition is the analogue of the key theorem for $\Phi_{\delta}$ and the discrete interface.

Proposition 9. Let $\left(\gamma_{\delta}(n)\right)_{n>0}$ have the law of the interface emanating at a in the critical Ising model on $\left(D_{\delta}, a, b, c\right)$ with dipolar boundary conditions, parametrized by the number of steps. For any $z \in[a, b]$, we have that

$$
\left(\Phi_{\delta}\left(D_{\delta} \backslash \gamma_{\delta}[0, n], r, \ell, \gamma_{\delta}(n), z\right)\right)_{n \wedge \tau(z)}
$$

is a discrete time martingale, where

$$
\tau(z):=\inf \left\{n: \gamma_{\delta}(n) \in[z, \ell]\right\} .
$$

See Section 6.2 for a precise definition of $D_{\delta} \backslash \gamma_{\delta}[0, n]$. The proof of Proposition 9. which is in essence combinatorial, is also given in Section 6.2 .

The key theorem, which is the martingale property of $\Phi$ for the subsequential scaling limits of $\left(\gamma_{\delta}\right)_{\delta>0}$ is hence a consequence of the following observable convergence theorem, as it is inherited from discrete level:

Theorem 10. If $\left(\Omega_{\delta}, r_{\delta}, \ell_{\delta}, x_{\delta}, z_{\delta}\right) \rightarrow(\Omega, r, \ell, x, z)$ as $\delta \rightarrow 0$, then

$$
\frac{1}{\sqrt{\delta}} \Phi_{\delta}\left(\Omega_{\delta}, r_{\delta}, \ell_{\delta}, x_{\delta}, z_{\delta}\right) \underset{\delta \rightarrow 0}{\longrightarrow} \Phi(\Omega, r, \ell, x, z) .
$$

The convergence is locally uniform with respect to the domains.

We are now in position to prove the key theorem:

Proof of Theorem 6. By Proposition 9, $\Phi_{\delta}$ is a discrete martingale with respect to the Ising interface, when parametrized by the lattice steps. Since $\frac{1}{\sqrt{\delta}} \Phi_{\delta}$ converges to $\Phi$ as $\delta \rightarrow 0$, we deduce that $t \mapsto \Phi(D \backslash \gamma[0, t], r, \ell, \gamma(t), z)$ is a local martingale. The time continuity follows from the continuity of $\Phi$ with respect to the domain. 
The heart of the matter, namely the connection between discrete and continuous worlds, is therefore contained in Theorem 10, the proof thereof exploits special features of the Ising model, both combinatorial and analytical. It is discussed in the following subsections.

5.2. Four ingredients. In this subsection, we give four propositions, which together allow to deduce the observable convergence theorem (Theorem 10), as will be explained in Section 5.3

5.2.1. Correlation function representation. The first proposition allows for a representation of $\Phi_{\delta}$ in terms of discrete correlation functions on a dual Ising model:

Proposition 11. If we consider the Ising model on the vertices of $\left(\Omega_{\delta}, r, \ell, x, z\right)$ with + boundary condition on the arc $[r, \ell]$ and free boundary condition on the arc $[\ell, r]$, we have

$$
\frac{\mathbb{E}_{\Omega_{\delta}}^{[r, \ell]_{+}}\left[\sigma_{x} \sigma_{z}\right]}{\mathbb{E}_{\Omega_{\delta}}^{[r, \ell]_{+}}\left[\sigma_{x}\right]}=\Phi_{\delta}\left(\Omega_{\delta}, r, \ell, x, z\right) .
$$

The proof is given in Section 7

5.2.2. FK representation. The second proposition makes use of the Fortuin-Kasteleyn $(\mathrm{FK})$ representation of the dual Ising model (defined in Section 8.1) to give an expression for $\Phi_{\delta}\left(\Omega_{\delta}, r_{\delta}, \ell_{\delta}, x_{\delta}, z_{\delta}\right)$ in terms of expectations over FK interfaces of simple correlation functions:

Proposition 12. We have

$$
\Phi_{\delta}\left(\Omega_{\delta}, r, \ell, x, z\right)=\mathrm{E}_{\delta}^{\mathrm{A}}\left(\Omega_{\delta}, r, \ell, x, z\right)+\mathrm{E}_{\delta}^{\mathrm{B}}\left(\Omega_{\delta}, r, \ell, x, z\right),
$$

where

$$
\begin{aligned}
\mathrm{E}_{\delta}^{\mathrm{A}}\left(\Omega_{\delta}, r, \ell, x, z\right) & :=\frac{\mathbb{E}_{\delta}^{\mathrm{A}}\left[\mathbb{E}_{\Omega_{\delta} \backslash \lambda_{\delta}}^{\text {free }}\left[\sigma_{x} \sigma_{z}\right]\right]}{\mathbb{E}_{\Omega_{\delta}}^{[r, \ell]_{+}}\left[\sigma_{x}\right]}, \\
\mathrm{E}_{\delta}^{\mathrm{B}}\left(\Omega_{\delta}, r, \ell, x, z\right) & :=\mathbb{E}_{\delta}^{\mathrm{B}}\left[\mathbb{E}_{\Omega_{\delta} \backslash \tilde{\lambda}_{\delta}}^{[r, x]_{+}}\left[\sigma_{z}\right]\right]
\end{aligned}
$$

where

- the expectation $\mathbb{E}_{\delta}^{\mathrm{A}}$ is taken over the realizations $\lambda_{\delta}$ of a critical FK-Ising interface in $\Omega_{\delta}$ from $\ell$ to $r$;

- the expectation $\mathbb{E}_{\delta}^{\mathrm{B}}$ is taken over all realizations $\tilde{\lambda}_{\delta}$ of a critical FK interface in $\Omega_{\delta}$ from $\ell$ to $r$, conditioned to pass through $x$, stopped at $x$;

- the correlation $\mathbb{E}_{\Omega_{\delta} \backslash \lambda_{\delta}}^{\text {free }}\left[\sigma_{x} \sigma_{z}\right]$ is the correlation of the spins at $x$ and $z$ of the critical Ising model on $\Upsilon_{\delta}$ with free boundary conditions, where $\Upsilon_{\delta}$ is the connected component of $\Omega_{\delta} \backslash \lambda_{\delta}$ (the graph $\Omega_{\delta}$ with the edges crossed by $\lambda_{\delta}$ removed) containing $x$ and $z$;

- the correlation $\mathbb{E}_{\Omega_{\delta} \backslash \tilde{\lambda}_{\delta}}^{[r, x]_{+}}\left[\sigma_{z}\right]$ is the magnetization at $z$ of the critical Ising model on the connected component of $\Omega_{\delta} \backslash \tilde{\lambda}_{\delta}$ that contains $z$, with + boundary condition on $[r, x]$ and free boundary condition on $[x, r]$.

The proof of this proposition, as well as the definition of the FK model and of its interface, are given in Section 8 . 
5.2.3. From discrete to continuum. Let us now introduce the continuous analogues of the discrete objects appearing in Proposition 12 the correlation functions and the curves. The convergence of the discrete correlation functions to the continuous ones is dealt with in Section 9 .

Definition 13. If $\Omega$ is a simply connected domain we define the correlation functions $\left\langle\sigma_{x} \sigma_{z}\right\rangle_{\Omega}^{\text {free }}$ and $\left\langle\sigma_{x}\right\rangle_{\Omega}^{[r, \ell]_{+}}$by

$$
\begin{aligned}
\left\langle\sigma_{x} \sigma_{z}\right\rangle_{\Omega}^{\text {free }} & :=\left|\eta^{\prime}(x)\right|^{\frac{1}{2}}\left|\eta^{\prime}(y)\right|^{\frac{1}{2}}\left\langle\sigma_{\eta(x)} \sigma_{\eta(y)}\right\rangle_{\mathbb{H}}^{\text {free }} \\
\left\langle\sigma_{\eta_{2}} \sigma_{\eta_{1}}\right\rangle_{\mathbb{H}}^{\text {free }} & :=\frac{\sqrt{2}+1}{\pi} \frac{1}{\left|\eta_{1}-\eta_{2}\right|} \\
\left\langle\sigma_{x}\right\rangle_{\Omega}^{[r, \ell]_{+}} & :=\left|\eta^{\prime}(x)\right|^{\frac{1}{2}}\left\langle\sigma_{\eta(x)}\right\rangle_{\mathbb{H}}^{[\eta(r), \eta(\ell)]_{+}}, \\
\left\langle\sigma_{\eta_{1}}\right\rangle_{\mathbb{H}}^{\left[\eta_{\infty}, \eta_{0}\right]_{+}} & :=\sqrt{\frac{\sqrt{2}+1}{2 \pi}} \sqrt{\frac{\left|\eta_{\infty}-\eta_{0}\right|}{\left|\eta_{1}-\eta_{0}\right|\left|\eta_{1}-\eta_{\infty}\right|}} .
\end{aligned}
$$

for any conformal mapping $\eta: \Omega \rightarrow \mathbb{H}$ and any $x, y, r, \ell \in \partial \Omega$, provided the right hand sides are well-defined (these definitions are independent of the choice of $\eta$ ).

The FK-Ising interfaces converge to variants of SLE(16/3) that are defined in Section 10.

The third proposition gives the convergence of the discrete expectations $\mathrm{E}_{\delta}^{\mathrm{A}}$ and $\mathrm{E}_{\delta}^{\mathrm{B}}$ as $\delta \rightarrow 0$ to continuous expectations. The definitions of chordal $\operatorname{SLE}(\kappa)$ and $\operatorname{SLE}(\kappa ; \rho)$ are given in Section 10 .

Proposition 14. As $\delta \rightarrow 0$ and $\left(\Omega_{\delta}, r_{\delta}, \ell_{\delta}, x_{\delta}, z_{\delta}\right) \rightarrow(\Omega, r, \ell, x, z)$, we have

$$
\begin{aligned}
& \frac{1}{\sqrt{\delta}} \mathrm{E}_{\delta}^{\mathrm{A}}\left(\Omega_{\delta}, r_{\delta}, \ell_{\delta}, x_{\delta}, z_{\delta}\right) \underset{\delta \rightarrow 0}{\longrightarrow} \mathrm{E}^{\mathrm{A}}(\Omega, r, \ell, x, z) \\
& \frac{1}{\sqrt{\delta}} \mathrm{E}_{\delta}^{\mathrm{B}}\left(\Omega_{\delta}, r_{\delta}, \ell_{\delta}, x_{\delta}, z_{\delta}\right) \underset{\delta \rightarrow 0}{\longrightarrow} \mathrm{E}^{\mathrm{B}}(\Omega, r, \ell, x, z),
\end{aligned}
$$

where the continuous expectations $\mathrm{E}^{\mathrm{A}}(\Omega, r, \ell, x, z)$ and $\mathrm{E}^{\mathrm{B}}(\Omega, r, \ell, x, z)$ are defined by

$$
\begin{aligned}
& \mathrm{E}^{\mathrm{A}}(\Omega, r, \ell, x, z):=\mathbb{E}^{\mathrm{A}}\left[\frac{\left\langle\sigma_{x} \sigma_{z}\right\rangle_{\Omega \backslash \lambda}^{\text {free }}}{\left\langle\sigma_{x}\right\rangle_{\Omega}^{[r, \ell]_{+}}}\right], \\
& \mathrm{E}^{\mathrm{B}}(\Omega, r, \ell, x, z):=\mathbb{E}^{\mathrm{B}}\left[\left\langle\sigma_{z}\right\rangle_{\Omega \backslash \tilde{\lambda}}^{[r, \ell]_{+}}\right],
\end{aligned}
$$

where

- the correlation functions are as in Definition 13.

- the expectation $\mathbb{E}^{\mathrm{A}}$ is over the realizations $\lambda$ of a chordal SLE(16/3) trace from $\ell$ to $r$.

- the expectation $\mathbb{E}^{\mathrm{B}}$ is over the realizations $\tilde{\lambda}$ of an $S L E(16 / 3 ;-8 / 3)$ trace starting from $\ell$, with observation point $r$ and force point $x$, stopped upon hitting $x$.

- the integrand in $\mathbb{E}^{\mathrm{A}}$ is defined as 0 if $x$ and $z$ are in different connected components of $\Omega \backslash \lambda$.

The convergence is locally uniform with respect to the domains. 
The proof is given in Section 11 .

Remark 15. Notice that the ratio

$$
\frac{\left\langle\sigma_{x} \sigma_{z}\right\rangle_{\Omega \backslash \lambda}^{\text {free }}}{\left\langle\sigma_{x}\right\rangle_{\Omega}^{[r, \ell]_{+}}}
$$

is well-defined even if $x$ lies on a rough part of $\partial \Omega$; on the other hand, recall that we assumed that $z$ is on a vertical part of $\partial \Omega$.

5.2.4. Computations in the continuum. The fourth and last proposition is the explicit computation of $\mathrm{E}^{\mathrm{A}}$ and $\mathrm{E}^{\mathrm{B}}$ :

Proposition 16. We have

$$
\begin{aligned}
\mathrm{E}^{\mathrm{A}}(\Omega, r, \ell, x, z) & =\left|\eta_{\Omega}^{\prime}(z)\right|^{\frac{1}{2}} \mathrm{E}^{\mathrm{A}}\left(\mathbb{H}, \eta_{\Omega}(r), \eta_{\Omega}(\ell), \eta_{\Omega}(x), \eta_{\Omega}(z)\right) \\
\mathrm{E}^{\mathrm{B}}(\Omega, r, \ell, x, z) & =\left|\eta_{\Omega}^{\prime}(z)\right|^{\frac{1}{2}} \mathrm{E}^{\mathrm{B}}\left(\mathbb{H}, \eta_{\Omega}(r), \eta_{\Omega}(\ell), \eta_{\Omega}(x), \eta_{\Omega}(z)\right) \\
\Phi(\Omega, r, \ell, x, z) & =\mathrm{E}^{\mathrm{A}}(\Omega, r, \ell, x, z)+\mathrm{E}^{\mathrm{B}}(\Omega, r, \ell, x, z)
\end{aligned}
$$

for any conformal mapping $\eta_{\Omega}: \Omega \rightarrow \mathbb{H}$. On $\mathbb{H}$, we have, if $\eta_{\infty}<\eta_{0}<\eta_{1}<\eta_{2}$,

$$
\begin{aligned}
\mathrm{E}^{\mathrm{A}}\left(\mathbb{H}, \eta_{\infty}, \eta_{0}, \eta_{1}, \eta_{2}\right) & =C_{\mathrm{A}} \frac{\left(\eta_{1}-\eta_{\infty}\right)^{1 / 2}}{\left(\eta_{2}-\eta_{\infty}\right)^{1 / 2}\left(\eta_{2}-\eta_{1}\right)^{1 / 2}} \int_{\chi}^{1} \frac{(1-\zeta)}{\zeta^{3 / 4}(\zeta-\chi)^{3 / 4}} \mathrm{~d} \zeta \\
\mathrm{E}^{\mathrm{B}}\left(\mathbb{H}, \eta_{\infty}, \eta_{0}, \eta_{1}, \eta_{2}\right) & =C_{\mathrm{B}} \frac{\left(\eta_{1}-\eta_{\infty}\right)^{1 / 2}}{\left(\eta_{2}-\eta_{\infty}\right)^{1 / 2}\left(\eta_{2}-\eta_{1}\right)^{1 / 2}} \cdot \frac{1}{\sqrt{\chi}} \int_{1-\chi}^{1} \frac{\zeta^{1 / 2}}{(\zeta-1+\chi)^{1 / 4}(1-\zeta)^{1 / 4}} \mathrm{~d} \zeta
\end{aligned}
$$

where the cross-ratio $\chi$ is defined by

$$
\chi:=\frac{\eta_{0}-\eta_{\infty}}{\eta_{2}-\eta_{0}} \frac{\eta_{2}-\eta_{1}}{\eta_{1}-\eta_{\infty}}
$$

and the constants $C_{\mathrm{A}}$ and $C_{\mathrm{B}}$ are:

$$
\begin{aligned}
C_{\mathrm{A}} & :=\frac{\sqrt{2 \sqrt{2}+2}}{\pi} \frac{\Gamma\left(\frac{3}{4}\right)}{\Gamma\left(\frac{1}{4}\right)}, \\
C_{\mathrm{B}} & :=\frac{2 \sqrt{1+\sqrt{2}}}{\pi^{3 / 2}}
\end{aligned}
$$

5.3. Convergence of the discrete martingale observable. From the four propositions of the previous subsection, we obtain the proof of Theorem 10 :

- By Proposition 11, we can represent $\Phi_{\delta}$ as a ratio of discrete spin correlation functions of the dual Ising model.

- By Proposition 12, we can represent the discrete spin correlation functions as expectations of simple correlation functions computed on random domains determined by FK interfaces.

- By Proposition 14 the FK expectations, renormalized by $\frac{1}{\sqrt{\delta}}$, converge to SLE expectations.

- By Proposition 16, the sum of the two SLE expectations is equal to $\Phi$.

We hence deduce the theorem: $\frac{1}{\sqrt{\delta}} \Phi_{\delta}\left(\Omega_{\delta}, r_{\delta}, \ell_{\delta}, x_{\delta}, z_{\delta}\right) \rightarrow \Phi(\Omega, r, \ell, x, z)$ as $\delta \rightarrow 0$. 


\section{The discrete martingale property}

In this section, we prove the martingale property of the discrete observable.

6.1. Low-temperature expansion. Let us first give a graphical representation of the discrete observable $\Phi_{\delta}\left(\Omega_{\delta}, r, \ell, x, z\right)$ defined in Section 5.1 as

$$
\frac{\tilde{\mathcal{Z}}\left(\Omega_{\delta}, r, \ell, x, z\right)}{\mathcal{Z}\left(\Omega_{\delta}, r, \ell, x\right)}
$$

where the denominator is the partition function of the critical Ising model with dipolar boundary conditions and the numerator is the partition function of the critical Ising model with modified boundary conditions (see Definition 8).

We call a collection of edges of $\Omega_{\delta}$ a contour. The following lemma gives a contour representation of $\Phi_{\delta}$, known as low-temperature expansion:

Lemma 17. We have

$$
\frac{\tilde{\mathcal{Z}}\left(\Omega_{\delta}, r, \ell, x, z\right)}{\mathcal{Z}\left(\Omega_{\delta}, r, \ell, x\right)}=\frac{\sum_{\tilde{\omega} \in \tilde{\mathcal{C}}} \alpha^{|\tilde{\omega}|}}{\sum_{\omega \in \mathcal{C}} \alpha^{|\omega|}},
$$

where $\alpha=\exp \left(-2 \beta_{c}\right)=\sqrt{2}-1$ and where

- $\mathcal{C}$ is the set of contours arising as interfaces (between + and - spins) of the configuration of spins appearing in $\mathcal{Z}\left(\Omega_{\delta}, r, \ell, x\right)$ (treating the spins on $[\ell, x]$ as - and the spins on $[x, r]$ as +$): \mathcal{C}$ is the set of contours $\omega$ such that each vertex of $\Omega_{\delta} \backslash(\{x\} \cup[r, \ell])$ belongs to an even number of edges of $\omega$ and such that $x$ belongs to an odd number of edges of $\omega$.

- $\tilde{\mathcal{C}}$ is the set of contours arising as interfaces of the spin configurations appearing in $\tilde{\mathcal{Z}}\left(\Omega_{\delta}, r, \ell, x, z\right)$ (treating the spins on $[\ell, x] \cup[z, r]$ as - and the spins on $[x, z]$ as +$): \tilde{\mathcal{C}}$ is the set of contours $\tilde{\omega}$ such that each vertex of $\Omega_{\delta} \backslash(\{x, z\} \cup[r, \ell])$ belongs to an even number of edges of $\tilde{\omega}$ and such that $x$ and $z$ belong to an odd number of edges of $\tilde{\omega}$.

Proof. Let $\mathcal{E}$ denote the set of edges of $\Omega_{\delta}$ that are not on $[r, \ell]$.

By definition of the contour sets as interfaces between the spins, each edge $e \in \mathcal{E}$ present in a configuration $\omega \in \mathcal{C}$ corresponds to a pair of adjacent spins $i \sim j$ that are of opposite signs and hence the contribution $-\sigma_{i} \sigma_{j}$ to the energy (defined by Equation 2.1) of that pair of spins is +1 (the pairs of spins possibly include the spins on $[\ell, x]$ and the ones on $[x, r])$. On the other hand, each vacant edge in a configuration $\omega \in \mathcal{C}$ (i.e. each edge of $\Omega_{\delta}$ that does not belong to $\omega$ ) corresponds to a pair of adjacent spins that have the same sign, and the corresponding contribution of that pair to the energy is -1 .

As the number of pairs we are summing over is $|\mathcal{E}|$ (the number of elements in $\mathcal{E}$ ), we have that the energy $\mathbf{H}(\sigma)$ of a spin configuration $\sigma$ corresponding to a contour $\omega \in \mathcal{C}$ is equal to $2|\omega|-|\mathcal{E}|$. By exactly the same considerations, the energy of a spin configuration $\tilde{\sigma}$ corresponding to a contour $\tilde{\omega} \in \tilde{\mathcal{C}}$ is equal to $2|\tilde{\omega}|-|\mathcal{E}|$. Hence, we obtain

$$
\begin{aligned}
\frac{\tilde{\mathcal{Z}}\left(\Omega_{\delta}, r, \ell, x, z\right)}{\mathcal{Z}\left(\Omega_{\delta}, r, \ell, x\right)} & =\frac{\sum_{\tilde{\omega} \in \tilde{\mathcal{C}}} \exp (-\beta(2|\tilde{\omega}|-|\mathcal{E}|))}{\sum_{\omega \in \mathcal{C}} \exp (-\beta(2|\omega|-|\mathcal{E}|))} \\
& =\frac{\sum_{\tilde{\omega} \in \tilde{\mathcal{C}}} \alpha^{|\tilde{\omega}|}}{\sum_{\omega \in \mathcal{C}} \alpha^{|\omega|}} .
\end{aligned}
$$




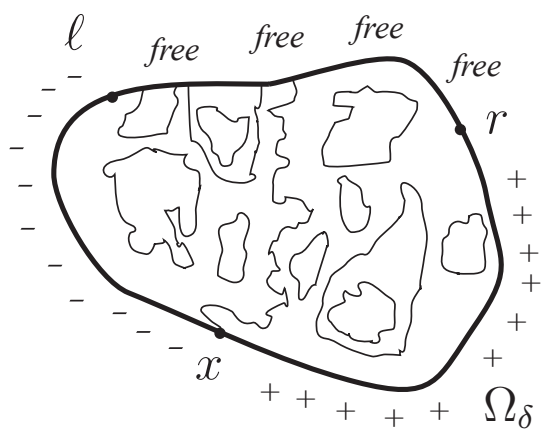

FigurE 6.1. Schematic sketch of the contours in $\mathcal{C}$.
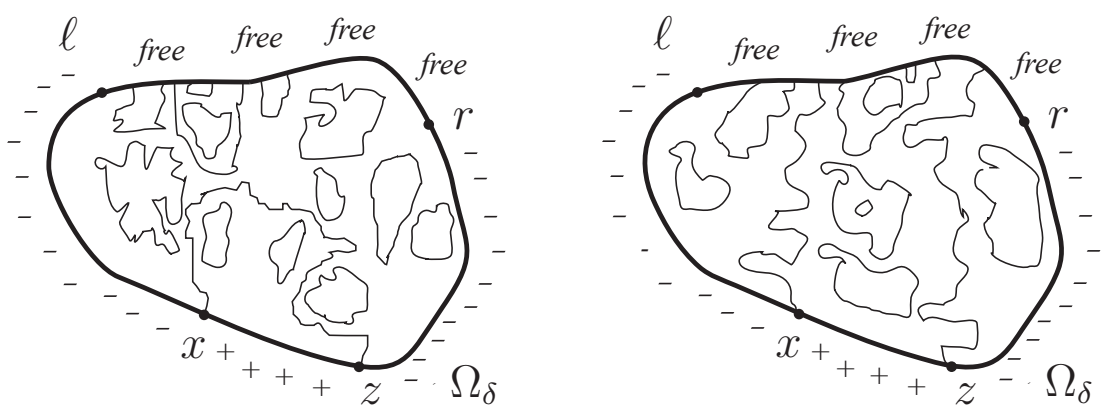

Figure 6.2. Schematic sketch of the contours in $\tilde{\mathcal{C}}$.

Remark 18. The contours in $\mathcal{C}$ contain (possibly non-simple) loops, with an interface from $x$ to $[r, \ell]$ and possibly arcs pairing vertices of $[r, \ell]$ (see Figure 6.1). The contours in $\tilde{\mathcal{C}}$ contain loops and arcs, plus either an interface from $x$ to $z$ or two interfaces, emanating at $x$ and $z$, and both ending on $[r, \ell]$ (see Figure 6.2).

6.2. Proof of Proposition 9. Thanks to the low-temperature expansion detailed in the previous subsection, we can now prove the discrete martingale property:

Proposition (Proposition 9). Let $\left(\gamma_{\delta}(n)\right)_{n>0}$ have the law of the interface arising at $b$ in the critical Ising model on $\left(D_{\delta}, r, \ell, b\right)$ with dipolar boundary conditions, parametrized by the number of steps. For any $z \in[b, r]$,

$$
\left(\Phi_{\delta}\left(D_{\delta} \backslash \gamma_{\delta}[0, n], r, \ell, \gamma_{\delta}(n), z\right)\right)_{n \wedge \tau(z)}
$$

is a discrete time martingale, where

$$
\tau(z):=\inf \left\{n: \gamma_{\delta}(n) \in[z, \ell]\right\} .
$$

The discrete domain $D_{\delta} \backslash \gamma_{\delta}[0, n]$ is defined as the connected component of

$$
D_{\delta} \backslash\left(\gamma_{\delta}^{\text {Left Side }} \cup \gamma_{\delta}^{\text {Right Side }}\right)
$$

that contains $r, \ell$ and $z$, where 
- $\gamma_{\delta}^{\text {Left Side }}$ is the set of faces of $D_{\delta}$ that either share an edge or a vertex with the left side of $\gamma_{\delta}[0, n-1]$;

- $\gamma_{\delta}^{\text {Right Side }}$ is the set of faces of $D_{\delta}$ that share an edge with the right side of $\gamma_{\delta}[0, n]$.

Proof of Proposition 9. We prove that, conditionally on the event that $0 \leq n<\tau(z)$ (if $n \geq \tau(z)$, there is nothing to prove):

$\mathbb{E}\left[\Phi_{\delta}\left(D_{\delta} \backslash \gamma_{\delta}[0, n+1], r, \ell, \gamma_{\delta}(n+1), z\right) \mid \gamma_{\delta}[0, n]\right]=\Phi_{\delta}\left(D_{\delta} \backslash \gamma_{\delta}[0, n], r, \ell, \gamma_{\delta}(n), z\right)$

By Lemma 17, we obtain

$$
\frac{\tilde{\mathcal{Z}}\left(D_{\delta} \backslash \gamma_{\delta}[0, n], r, \ell, \gamma_{\delta}(n), z\right)}{\mathcal{Z}\left(D_{\delta} \backslash \gamma_{\delta}[0, n], r, \ell, \gamma_{\delta}(n)\right)}=\frac{\sum_{\omega \in \tilde{\mathcal{C}}} \alpha^{|\omega|}}{\sum_{\omega \in \mathcal{C}} \alpha^{|\omega|}},
$$

where $\alpha=\sqrt{2}-1$ and $\mathcal{C}, \tilde{\mathcal{C}}$ are as in Lemma 17 (where the domain $\Omega_{\delta}$ is now $\left.D_{\delta} \backslash \gamma_{\delta}[0, n]\right)$.

Let $L, S, R$ be the three vertices adjacent that are the possible values for $\gamma_{\delta}(n+1)$, if the interface turns left, goes straight or turns right after $\gamma_{\delta}(n)$ (see Figure 6.3) and let $e_{L}, e_{S}, e_{R}$ be the edges from $\gamma_{\delta}(n)$ to $L, S, R$. Every contour configuration in $\mathcal{C}$ or $\tilde{\mathcal{C}}$ contains an interface emanating from $\gamma_{\delta}(n)$, and we hence split $\mathcal{C}$ as $\mathcal{C}_{L} \cup \mathcal{C}_{S} \cup \mathcal{C}_{R}$ and $\tilde{\mathcal{C}}$ as $\tilde{\mathcal{C}}_{L} \cup \tilde{\mathcal{C}}_{S} \cup \tilde{\mathcal{C}}_{R}$, where

$$
\begin{array}{rll}
\mathcal{C}_{L}:=\left\{\omega \in \mathcal{C}: e_{L} \in \omega\right\}, & \mathcal{C}_{S}:=\left\{\omega \in \mathcal{C}: e_{S} \in \omega, e_{L} \notin \omega\right\}, & \mathcal{C}_{R}:=\left\{\omega \in \mathcal{C}: e_{R} \in \omega, e_{L} \notin \omega\right\}, \\
\tilde{\mathcal{C}}_{L}:=\left\{\omega \in \tilde{\mathcal{C}}: e_{L} \in \omega\right\}, & \tilde{\mathcal{C}}_{S}:=\left\{\omega \in \tilde{\mathcal{C}}: e_{S} \in \omega, e_{L} \notin \omega\right\}, & \tilde{\mathcal{C}}_{R}:=\left\{\omega \in \tilde{\mathcal{C}}: e_{R} \in \omega, e_{L} \notin \omega\right\} .
\end{array}
$$

Notice that the slight asymmetry of these definitions follows our convention of turning left whenever there is an ambiguity. Writing

$$
\mathcal{Z}_{L}:=\sum_{\omega \in \mathcal{C}_{L}} \alpha^{|\omega|}, \quad \tilde{\mathcal{Z}}_{L}:=\sum_{\omega \in \tilde{\mathcal{C}}_{L}} \alpha^{|\omega|}, \quad \mathcal{Z}_{S}:=\sum_{\omega \in \mathcal{C}_{S}} \alpha^{|\omega|}, \ldots
$$

we get $\mathcal{Z}=\mathcal{Z}_{L}+\mathcal{Z}_{S}+\mathcal{Z}_{R}$ and $\tilde{\mathcal{Z}}=\tilde{\mathcal{Z}}_{L}+\tilde{\mathcal{Z}}_{S}+\tilde{\mathcal{Z}}_{R}$ and it is easy to check that we have

$$
\begin{aligned}
& \mathbf{P}_{\text {Left }}:=\mathbb{P}\left[\gamma_{\delta}(n+1)=L \mid \gamma_{\delta}[0, n]\right]=\frac{\mathcal{Z}_{L}}{\mathcal{Z}}, \\
& \mathbf{P}_{\text {Straight }}:=\mathbb{P}\left[\gamma_{\delta}(n+1)=S \mid \gamma_{\delta}[0, n]\right]=\frac{\mathcal{Z}_{S}}{\mathcal{Z}}, \\
& \mathbf{P}_{\text {Right }}:=\mathbb{P}\left[\gamma_{\delta}(n+1)=R \mid \gamma_{\delta}[0, n]\right]=\frac{\mathcal{Z}_{R}}{\mathcal{Z}} .
\end{aligned}
$$

Now remark that

$$
\Phi_{\delta}\left(D \backslash \gamma_{\delta}[0, n+1]\right)= \begin{cases}\frac{\tilde{\mathcal{Z}}_{L}}{\mathcal{Z}_{L}} & \text { on event } L, \\ \frac{\tilde{\mathcal{Z}}_{S}}{\mathcal{Z}_{S}} & \text { on event } S, \\ \frac{\mathcal{Z}_{R}}{\mathcal{Z}_{R}} & \text { on event } R\end{cases}
$$




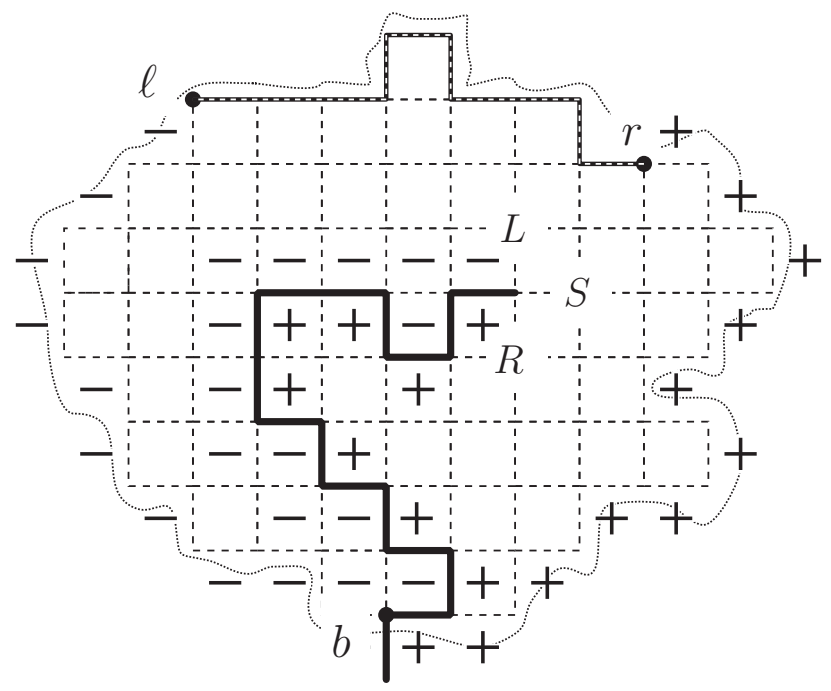

FiguRE 6.3.

The martingale property is thus verified by the following calculation:

$$
\begin{aligned}
& \mathbb{E}\left[\Phi_{\delta}\left(D \backslash \gamma_{\delta}[0, n+1], r, \ell, \gamma_{\delta}(n+1), z\right) \mid \gamma_{\delta}[0, n]\right] \\
= & \mathbf{P}_{\text {Left }} \frac{\tilde{\mathcal{Z}}_{L}}{\mathcal{Z}_{L}}+\mathbf{P}_{\text {Straight }} \frac{\tilde{\mathcal{Z}}_{S}}{\mathcal{Z}_{S}}+\mathbf{P}_{\text {Right }} \frac{\tilde{\mathcal{Z}}_{R}}{\mathcal{Z}_{R}} \\
= & \frac{\mathcal{Z}_{L}}{\mathcal{Z}} \frac{\tilde{\mathcal{Z}}_{L}}{\mathcal{Z}_{L}}+\frac{\mathcal{Z}_{S}}{\mathcal{Z}} \frac{\tilde{\mathcal{Z}}_{S}}{\mathcal{Z}_{S}}+\frac{\mathcal{Z}_{R}}{\mathcal{Z}} \frac{\tilde{\mathcal{Z}}_{R}}{\mathcal{Z}_{R}}=\frac{\tilde{\mathcal{Z}}_{L}+\tilde{\mathcal{Z}}_{S}+\tilde{\mathcal{Z}}_{R}}{\mathcal{Z}} \\
= & \Phi_{\delta}\left(D \backslash \gamma_{\delta}[0, n], r, \ell, \gamma_{\delta}(n), z\right) .
\end{aligned}
$$

\section{Kramers-Wannier duality and spin correlations}

In this section, we use Kramers-Wannier duality KrWa41 to represent the observable $\Phi_{\delta}\left(\Omega_{\delta}, r, \ell, x, z\right)$ as a ratio of spin correlations on the vertices of $\Omega_{\delta}$ with dual boundary conditions. This section uses in a crucial way the fact that we are at the critical temperature. For more details about Kramers-Wannier duality, see [KaCe71], or [Pal07, Chapter 1].

To understand the quantity $\Phi_{\delta}\left(\Omega_{\delta}, r, \ell, x, z\right)$, which is a ratio of partition functions of Ising models living on the faces of $\Omega_{\delta}$ (as in Figure 6.3) we need to introduce a dual Ising model (see Figure 7.1), which lives on the vertices of $\Omega_{\delta}$ : it is defined exactly like before, with the probability of a spin configuration $\left(\sigma_{x}\right)_{x \in \mathcal{V}}$ proportional to $\exp (-\beta \mathbf{H}(\sigma))$, where

$$
\mathbf{H}(\sigma):=-\sum_{x \sim y} \sigma_{x} \sigma_{y},
$$

the sum being over all pairs of adjacent vertices. The boundary conditions that we will need are somewhat simpler: we simply condition the vertices on the $\operatorname{arc}[r, \ell]$ to +1 , and let free the spins on the arc $[\ell, r] \backslash\{\ell, r\}$ (which we will denote $[\ell, r]$ for convenience). 


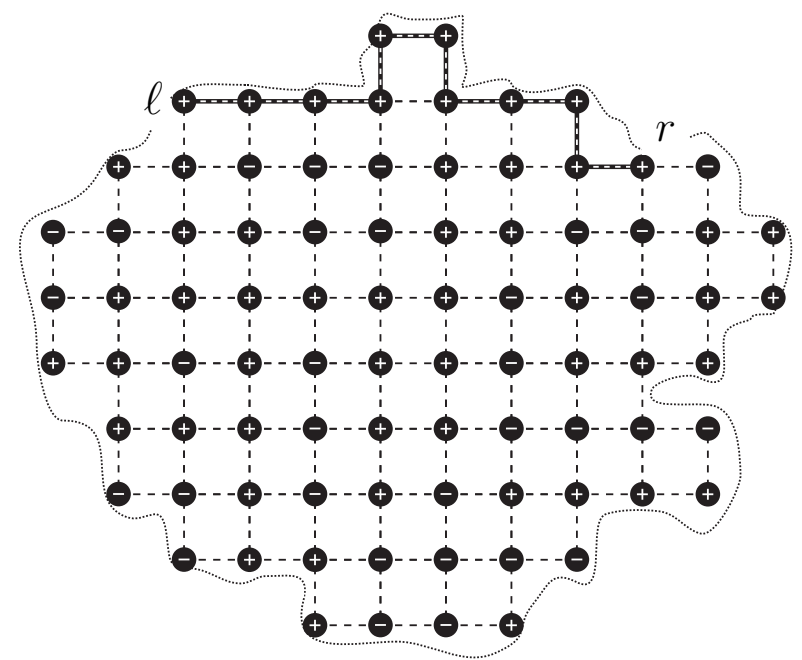

FIGURE 7.1. Dual Ising model living on the vertices of $\Omega_{\delta}$, with dual boundary conditions.

Proposition (Proposition 11). If we consider the Ising model on the vertices of the graph $\Omega_{\delta}$ with + boundary condition on the arc $[r, \ell]$ and free boundary condition on the arc $[\ell, r]$, we have

$$
\frac{\mathbb{E}_{\Omega_{\delta}}^{[r, \ell]_{+}}\left[\sigma_{x} \sigma_{z}\right]}{\mathbb{E}_{\Omega_{\delta}}^{[r, \ell]_{+}}\left[\sigma_{x}\right]}=\Phi_{\delta}\left(\Omega_{\delta}, r, \ell, x, z\right) .
$$

Proof. We use the Kramers-Wannier duality technique, also known as the hightemperature expansion of the Ising model. Starting from the left-hand side, we have

$$
\begin{aligned}
\frac{\mathbb{E}_{\Omega_{\delta}}^{[r, \ell]_{+}}\left[\sigma_{x} \sigma_{z}\right]}{\mathbb{E}_{\Omega_{\delta}}^{[r, \ell]_{+}}\left[\sigma_{x}\right]} & =\frac{\sum_{\sigma} \sigma_{x} \sigma_{z} \prod_{\langle i, j\rangle \in \mathcal{E}} \exp \left(\beta_{c} \sigma_{i} \sigma_{j}\right)}{\sum_{\sigma} \sigma_{x} \prod_{\langle i, j\rangle \in \mathcal{E}} \exp \left(\beta_{c} \sigma_{i} \sigma_{j}\right)} \\
& =\frac{\sum_{\sigma} \sigma_{x} \sigma_{z} \prod_{\langle i, j\rangle \in \mathcal{E}}\left(\cosh \beta_{c}+\sigma_{i} \sigma_{j} \sinh \beta_{c}\right)}{\sum_{\sigma} \sigma_{x} \prod_{\langle i, j\rangle \in \mathcal{E}}\left(\cosh \beta_{c}+\sigma_{i} \sigma_{j} \sinh \beta_{c}\right)} \\
& =\frac{\sum_{\sigma} \sigma_{x} \sigma_{z} \prod_{\langle i, j\rangle \in \mathcal{E}}\left(1+\alpha \sigma_{i} \sigma_{j}\right)}{\sum_{\sigma} \sigma_{x} \prod_{\langle i, j\rangle \in \mathcal{E}}\left(1+\alpha \sigma_{i} \sigma_{j}\right)} \\
& =\frac{\sum_{\sigma} \sigma_{x} \sigma_{z} \sum_{E \subset \mathcal{E}} \alpha^{|E|} \prod_{\langle i, j\rangle \in E} \sigma_{i} \sigma_{j}}{\sum_{\sigma} \sigma_{x} \sum_{E \subset \mathcal{E}} \alpha^{|E|} \prod_{\langle i, j\rangle \in E} \sigma_{i} \sigma_{j}} \\
& =\frac{\sum_{E \subset \mathcal{E}} \alpha^{|E|} \sum_{\sigma} \sigma_{x} \sigma_{z} \prod_{\langle i, j\rangle \in E} \sigma_{i} \sigma_{j}}{\sum_{E \subset \mathcal{E}} \alpha^{|E|} \sum_{\sigma} \sigma_{x} \prod_{\langle i, j\rangle \in E} \sigma_{i} \sigma_{j}} \\
& =\frac{\sum_{E \in \tilde{\mathcal{C}}} \alpha^{|E|}}{\sum_{E \in \mathcal{C}} \alpha^{|E|}} \\
& =\Phi_{\delta}\left(\Omega_{\delta}, r, \ell, x, z\right)
\end{aligned}
$$


For the second equation, we used that $\exp =\sinh +\cosh$, parity of $\sinh$ and cosh and $\sigma_{i} \sigma_{j}= \pm 1$. For the third one, we used that $\alpha=\sqrt{2}-1=\tanh \beta_{c}$. For the fourth, we expanded the product over all the edges.

The subtle point is the sixth; let us first look at the denominator. We used that if $E \subset \mathcal{E}$ is such that a vertex $v \in \Omega \backslash[r, \ell]$ arises an odd number of times in the product $\sigma_{x} \prod_{\langle i, j\rangle \in E} \sigma_{i} \sigma_{j}$, then the sum over all the spin configurations of this product vanishes by symmetry; hence the only $E \subset \mathcal{E}$ giving a nonzero contribution (which is then the number of possible spin configurations), are the contours in $\mathcal{C}$ (defined in Lemma 17): the vertex $x$ must belong to an odd number of edges, the vertices in $\Omega_{\delta} \backslash([r, \ell] \cup\{x\})$ must belong to an even number of edges and the vertices on the arc $[r, \ell]$ are free to belong to an arbitrary number of edges (since we are not summing over the spins at these vertices, which are set to +1 , because of the boundary condition). Hence, it is easy to see that the $E \subset \mathcal{E}$ which have nonzero contributions are precisely the $E \in \mathcal{C}$. Similarly, the terms giving a nonzero contribution to the numerators are the $E \subset \mathcal{E}$ such that the vertices $x$ and $z$ belong to an odd number of edges of $E$, the vertices in $\Omega_{\delta} \backslash([r, \ell] \cup\{x, z\})$ belong to an even number of edges of $E$ and the vertices in $[r, \ell]$ are free to belong to an arbitrary number of edges: those are precisely the contours in $\tilde{\mathcal{C}}$ (defined in Lemma 17 ).

\section{FK representation and connection events}

In the previous section, we showed (Proposition 11) that

$$
\Phi_{\delta}\left(\Omega_{\delta}, r, \ell, x, z\right)=\frac{\mathbb{E}_{\Omega_{\delta}}^{[r, \ell]_{+}}\left[\sigma_{x} \sigma_{z}\right]}{\mathbb{E}_{\Omega_{\delta}}^{[r, \ell]_{+}}\left[\sigma_{x}\right]} .
$$

In this section, we use the Fortuin-Kasteleyn representation of the Ising model to express the correlation functions of the right-hand side as a sum of correlation functions of simpler form (in the sense of boundary conditions) computed on random domains. Notice that this part does not use the fact that the temperature is critical.

8.1. The FK model. The Fortuin-Kasteleyn $(\mathrm{FK})$ model on a graph $\mathcal{G}=(\mathcal{V}, \mathcal{E})$ is a bond percolation model (i.e. a random subset of edges of $\mathcal{E}$ ) with two parameters $p \in[0,1]$ and $q \geq 0$, where the probability of an edge configuration $\omega \subset \mathcal{E}$ is proportional to

$$
\left(\frac{p}{1-p}\right)^{\# \operatorname{edges}(\omega)} q^{\# \operatorname{clusters}(\omega)},
$$

where the clusters of $\omega$ are the connected components of the graph $(\mathcal{V}, \omega)$ (the graph with vertices $\mathcal{V}$ and edges $\omega$ ). Given a (deterministic) subset $\mathfrak{b}$ of $\mathcal{V}$ (typically a part of the boundary of $\mathcal{V}$, if $\mathcal{V}$ is a domain) can introduce wired boundary condition, by declaring the vertices of $\mathfrak{b}$ to be in the same cluster (even if they are not linked by an edge in $\mathcal{E}$ ). When we do not wire boundary vertices, we call them free.

We will be interested in connection events for the FK model: for two vertices $a, b \in \mathcal{V}$, we will denote by $\{a \leadsto b\}$ the event that $a$ and $b$ belong to the same cluster of the FK configuration. We will denote, for $B \subset \mathcal{V}$, by $\{a \leadsto B\}$ the event that $\{a \leadsto b\}$ occurs for some $b \in B$. 
When $q=2$, the FK model provides a graphical representation of the Ising model at inverse temperature $\beta=\ln \left(\frac{1}{\sqrt{1-p}}\right)$ - the $p$ corresponding to $\beta_{c}=\frac{1}{2} \ln (\sqrt{2}+1)$ is hence $p_{c}=\frac{\sqrt{2}}{\sqrt{2}+1}$. In this paper, we will always assume that $q=2$, and we will only be interested in the $p=p_{c}$ case.

Theorem 19 (Fortuin-Kastelyn). Let $\omega \subset E$ have the law of an FK configuration on the domain $\left(\Omega_{\delta}, r, \ell\right)$ with parameters $p=1-e^{-2 \beta}$ and $q=2$, with wired boundary condition on $[r, \ell]$ and free boundary condition on $[\ell, r]$. Assign the spin value +1 to each of the vertices of the cluster containing $[r, \ell]$. For each other cluster of $\omega$, assign the same \pm 1 value to the spin at the vertices of this cluster, with probability $\frac{1}{2}-\frac{1}{2}$, independently of the other clusters. Then the law of the spin configuration $\sigma \in$ $\{ \pm 1\}^{\Omega_{\delta}}$ obtained via this procedure is that of an Ising model with inverse temperature $\beta,+$ boundary condition on $[r, \ell]$ and free boundary condition on $[\ell, r]$.

See [Gri06, Chapter 1] for a proof.

We call the FK model with $q=2$ the FK-Ising model, or just the FK model for short. When in addition $p=p_{c}$, we will refer to it as the critical FK-Ising model.

8.2. Correlation functions as connection probabilities. In this subsection, we use Theorem 19 to give an FK representation of the Ising spin correlations: the FKIsing model allows to understand how the influence between spins spreads through the graph.

Lemma 20. If we consider the Ising model (at any temperature) on $\left(\Omega_{\delta}, r, \ell\right)$ with + boundary condition on $[r, \ell]$ and free boundary condition on $[\ell, r]$ and the corresponding $F K$ model on $\left(\Omega_{\delta}, r, \ell\right)$ with wired boundary condition on $[r, \ell]$ and free boundary condition on $[\ell, r]$, then for any $x, z \in \Omega_{\delta}$ we have

$$
\begin{aligned}
& \mathbb{E}_{\Omega_{\delta}}^{[r, \ell]_{+}}\left[\sigma_{x}\right]=\mathbb{P}_{\Omega_{\delta}}^{[r, \ell]_{\mathrm{w}}}\{x \leftrightarrow[r, \ell]\} \\
& \mathbb{E}_{\Omega_{\delta}}^{[r, \ell]_{+}}\left[\sigma_{x} \sigma_{z}\right]=\mathbb{P}_{\Omega_{\delta}}^{[r, \ell]_{\mathrm{w}}}\{x \leadsto z\} \\
& =\mathbb{P}_{\Omega_{\delta}}^{[r, \ell]_{\mathrm{w}}}\{x \leftrightarrow z \leftrightarrow[r, \ell]\}+\mathbb{P}_{\Omega_{\delta}}^{[r, \ell]_{\mathrm{w}}}\{x \leftrightarrow z \leftrightarrow \mu[r, \ell]\}
\end{aligned}
$$

Proof. We use the FK representation of the Ising model (Theorem 19), sampling an Ising configuration from an FK-Ising configuration.

To obtain the first identity, it suffices to see that, conditionally on $\{x \leadsto[r, \ell]\}$, the spin $\sigma_{x}$ takes the value 1 (and hence is of expectation 1), and conditionally on $\{x$ th $\rightarrow r, \ell]\}$, the spin $\sigma_{x}$ takes the values -1 and +1 with equal probabilites (hence is of expectation 0 ).

To obtain the second identity, notice that, conditionally on $\{x \rightsquigarrow z\}$, the spins $\sigma_{x}$ and $\sigma_{z}$ are the same (and hence their expected product is 1 ), that conditionally on $\{x \leftrightarrow \mu r z\}$, they are independent (and since a centered \pm 1 is sampled for either $x$ or $z$ or both, the expected product is 0$)$.

8.3. Discrete vertex domains. We will need to consider graphs that are slightly more general than the discrete domains defined in Section 2.2. Let $\mathbb{C}_{\delta}$ be the square grid of mesh size $\delta$.

- We call a subgraph $\Omega_{\delta}$ of $\mathbb{C}_{\delta}$ a discrete vertex domain if it is a connected and simply connected (i.e. each face of $\Omega_{\delta}$ is a face of $\mathbb{C}_{\delta}$ ). 


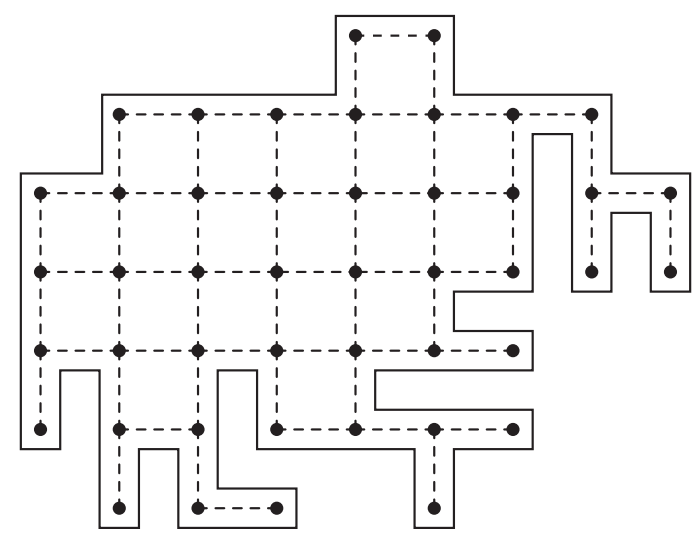

FiguRE 8.1. Discrete vertex domain.

- We denote by $\partial \Omega_{\delta}$ the Jordan curve that lives between $\Omega_{\delta}$ and the complementary of its dual (see Figure 8.1).

- When needed, we will identify $\Omega_{\delta}$ with the Jordan domain bounded by $\partial \Omega_{\delta}$.

- We denote by $\partial_{0} \Omega_{\delta}$ the set of vertices of $\Omega_{\delta}$ at distance less than $\frac{\delta}{2}$ to the curve $\partial \Omega_{\delta}$.

- We identify any given arc $[v, w] \subset \partial \Omega_{\delta}$ with the vertices of $\partial_{0} \Omega_{\delta}$ at distance less than $\frac{\delta}{2}$ to $[v, w]$.

- When there is no ambiguity, we identify the vertices of $\partial_{0} \Omega_{\delta}$ with the closest points of $\partial \Omega_{\delta}$.

Let us remark that all the discrete domains (as defined in Section 2.2) are discrete vertex domains, but that the converse is not true.

8.4. Interfaces, screening effects and random domains. The FK setup of Theorem 19 and Lemma 20, with wired boundary condition on an arc $[r, \ell]$ of a discrete domain $\left(\Omega_{\delta}, r, \ell\right)$ and free boundary condition on the other arc $[\ell, r]$ naturally generates an interface $\lambda_{\delta}$, which is the boundary of the FK cluster of the arc $[r, \ell]$, which links $r$ and $\ell$; we will always orient it from $\ell$ to $r$. The FK interface lives between $\Omega_{\delta}$ and its dual graph (see Figure 8.2 ) and is qualitatively very different from an Ising model interface: at critical temperature, its scaling limit is SLE(16/3).

The FK interface has convenient properties and is very well understood thanks to discrete complex analysis techniques introduced by Smirnov [Smi10a, Smi06], which is the reason why it plays a crucial role in our analysis. We will in particular make essential use of the following properties:

- The domain Markov property (also known as spatial Markov property): we have equivalences between:

(1) the conditional law of the FK model in $\left(\Omega_{\delta}, r, \ell\right)$, knowing the interface $\lambda_{\delta}[0, n]$ (or an initial segment of it);

(2) the law of the FK model on the connected components of $\Omega_{\delta} \backslash \lambda_{\delta}[0, n]$, with each connected component disconnected from $\lambda_{\delta}(n)$ having either purely wired or purely free boundary conditions, depending on whether it is on the left or the right of $\lambda_{\delta}[0, n]$. 


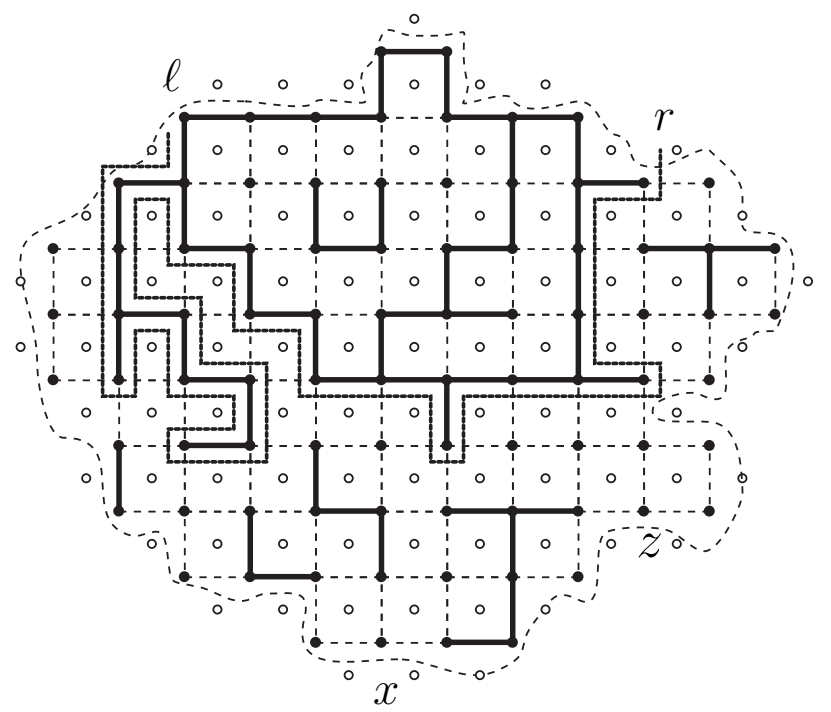

FiguRE 8.2. The FK interface

Hence, we can split a domain with mixed boundary conditions into a collection of random subdomains with simpler boundary conditions. The domain Markov property is a direct consequence of a fundamental screening property of the FK model, which asserts that the conditional law of an FK configuration outside of some domain can be encoded through boundary conditions (describing which boundary vertices are in the same cluster).

- The boundary hitting probabilities: the event that the FK interface $\lambda_{\delta}$ hits a point $y$ on the free arc $[\ell, r]$ (or more precisely: is such that $\lambda_{\delta} \cup[r, \ell]$ disconnects $y$ from $\infty$ ) is the same as the event that $\{y \leadsto[r, \ell]\}$ (i.e. that $y$ belongs to the same cluster as $[r, \ell])$. The boundary hitting probabilities can then be computed in the scaling limit thanks to discrete complex analysis results concerning this interface: the discrete holomorphic observable introduced in [Smi10a], which is complexified version of the passage probability, exactly gives this passage probability on the boundary of the domain.

From the above properties, we immediately deduce the following lemma, which will be instrumental in the next subsection:

Lemma 21. With the notation of Lemma 20, we have

$$
\begin{aligned}
\mathbb{P}_{\Omega_{\delta}}^{[r, \ell]_{\mathrm{w}}}\{x \rightsquigarrow[r, \ell]\} & =\mathbb{P}_{\Omega_{\delta}}^{[r, \ell]_{\mathrm{w}}}\left\{\lambda_{\delta} \text { passes at } x\right\} \\
\mathbb{P}_{\Omega_{\delta}}^{[r, \ell]_{\mathrm{w}}}\{x \rightsquigarrow z \rightsquigarrow[r, \ell]\} & =\mathbb{P}_{\Omega_{\delta}}^{[r, \ell]_{\mathrm{w}}}\left\{\lambda_{\delta} \text { passes at } x \text { and } z\right\}
\end{aligned}
$$

8.5. Proof of Proposition 12. Let us first recall Proposition 12 (see Figures 8.3 and 8.4.

Proposition (Proposition 12). We have

$$
\Phi_{\delta}\left(\Omega_{\delta}, r, \ell, x, z\right)=\mathrm{E}_{\delta}^{\mathrm{A}}\left(\Omega_{\delta}, r, \ell, x, z\right)+\mathrm{E}_{\delta}^{\mathrm{B}}\left(\Omega_{\delta}, r, \ell, x, z\right),
$$


where

$$
\begin{aligned}
\mathrm{E}_{\delta}^{\mathrm{A}}\left(\Omega_{\delta}, r, \ell, x, z\right) & :=\frac{\mathbb{E}_{\delta}^{\mathrm{A}}\left[\mathbb{E}_{\Omega_{\delta} \backslash \lambda_{\delta}}^{\text {free }}\left[\sigma_{x} \sigma_{z}\right]\right]}{\mathbb{E}_{\Omega_{\delta}}^{[r, \ell]_{+}}\left[\sigma_{x}\right]}, \\
\mathrm{E}_{\delta}^{\mathrm{B}}\left(\Omega_{\delta}, r, \ell, x, z\right) & :=\mathbb{E}_{\delta}^{\mathrm{B}}\left[\mathbb{E}_{\Omega_{\delta} \backslash \tilde{\lambda}_{\delta}}^{[r, x]_{+}}\left[\sigma_{z}\right]\right],
\end{aligned}
$$

where

- the expectation $\mathbb{E}_{\delta}^{\mathrm{A}}$ is taken over the realizations $\lambda_{\delta}$ of a critical FK-Ising interface in $\Omega_{\delta}$ from $\ell$ to $r$;

- the expectation $\mathbb{E}_{\delta}^{\mathrm{B}}$ is taken over all realizations $\tilde{\lambda}_{\delta}$ of a critical FK interface in $\Omega_{\delta}$ from $\ell$ to $r$, conditioned to pass through $x$, stopped at $x$;

- the correlation $\mathbb{E}_{\Omega_{\delta} \backslash \lambda_{\delta}}^{\text {free }}\left[\sigma_{x} \sigma_{z}\right]$ is the correlation of the spins at $x$ and $z$ of the critical Ising model on $\Upsilon_{\delta}$ with free boundary conditions, where $\Upsilon_{\delta}$ is the connected component of $\Omega_{\delta} \backslash \lambda_{\delta}$ (the graph $\Omega_{\delta}$ with the edges crossed by $\lambda_{\delta}$ removed) containing $x$ and $z$;

- the correlation $\mathbb{E}_{\Omega_{\delta} \backslash \tilde{\lambda}_{\delta}}^{[r, x]_{+}}\left[\sigma_{z}\right]$ is the magnetization at $z$ of the critical Ising model on the connected component of $\Omega_{\delta} \backslash \tilde{\lambda}_{\delta}$ that contains $z$, with + boundary condition on $[r, x]$ and free boundary condition on $[x, r]$.

Remark 22. The correlation $\mathbb{E}_{\Omega_{\delta} \backslash \lambda_{\delta}}^{\text {free }}\left[\sigma_{x} \sigma_{z}\right]$ is equal to 0 if $x$ and $z$ lie in two different connected components of $\Omega_{\delta} \backslash \lambda_{\delta}$.

Remark 23. The graphs $\Omega_{\delta} \backslash \lambda_{\delta}$ and $\Omega_{\delta} \backslash \tilde{\lambda}_{\delta}$ are discrete vertex domains; notice also that there is no ambiguity in the definition of the arc $[r, x]$ in $\Omega_{\delta} \backslash \tilde{\lambda}_{\delta}$ (see Figure 8.4 .

Proof. By Lemmas 20 and 21, we have

$$
\begin{aligned}
& \mathbb{E}_{\Omega_{\delta}}^{[r, \ell]_{+}}\left[\sigma_{x} \sigma_{z}\right] \\
= & \mathbb{P}_{\Omega_{\delta}}^{[r, \ell]_{\mathrm{w}}}\left[\{x \leftrightarrow z\} \cap\left\{\lambda_{\delta} \text { does not pass at } x \text { or } z\right\}\right] \\
& +\mathbb{P}_{\Omega_{\delta}}^{[r, \ell]_{\mathrm{w}}}\left\{\lambda_{\delta} \text { passes at } x \text { and } z\right\} .
\end{aligned}
$$

Because of the domain Markov property, we have that

$$
\begin{aligned}
& \mathbb{P}_{\Omega_{\delta}}^{[r, \ell]_{\mathrm{w}}}\left[\{x \rightsquigarrow z\} \cap\left\{\lambda_{\delta} \text { does not pass at } x \text { or } z\right\}\right] \\
= & \mathbb{E}_{\delta}^{\mathrm{A}}\left[\mathbb{P}_{\Omega_{\delta} \backslash \lambda_{\delta}}^{\text {free }}\{x \rightsquigarrow z\}\right],
\end{aligned}
$$

where $\mathbb{E}_{\delta}^{\mathrm{A}}$ is as in the statement of the proposition and the probability $\mathbb{P}_{\Omega_{\delta} \backslash \lambda_{\delta}}^{\text {free }}\{x \rightsquigarrow z\}$ is zero whenever $x$ and $z$ lie in two different components of $\Omega_{\delta} \backslash \lambda_{\delta}$ - this happens in particular when $\lambda_{\delta}$ passes at $x$ or $z$.

Also using domain Markov property, and the topological fact that the interface emanating at $\ell_{\delta}$ cannot hit $z_{\delta}$ before $x_{\delta}$, we obtain

$$
\begin{aligned}
& \frac{\mathbb{P}_{\Omega_{\delta}}^{[r, \ell]_{\mathrm{w}}}\left\{\lambda_{\delta} \text { passes at } x \text { and } z\right\}}{\mathbb{P}_{\Omega_{\delta}}^{[r, \ell]_{\mathrm{w}}}\left\{\lambda_{\delta} \text { passes at } x\right\}} \\
= & \mathbb{P}_{\Omega_{\delta}}^{[r, \ell]_{\mathrm{w}}}\left[\left\{\lambda_{\delta} \text { passes at } z\right\} \mid\left\{\lambda_{\delta} \text { passes at } x\right\}\right] \\
= & \mathbb{E}_{\delta}^{\mathrm{B}}\left[\mathbb{P}_{\Omega_{\delta} \backslash \tilde{\lambda}_{\delta}}^{[r, x]_{+}}\{z \leftrightarrow[r, x]\}\right],
\end{aligned}
$$




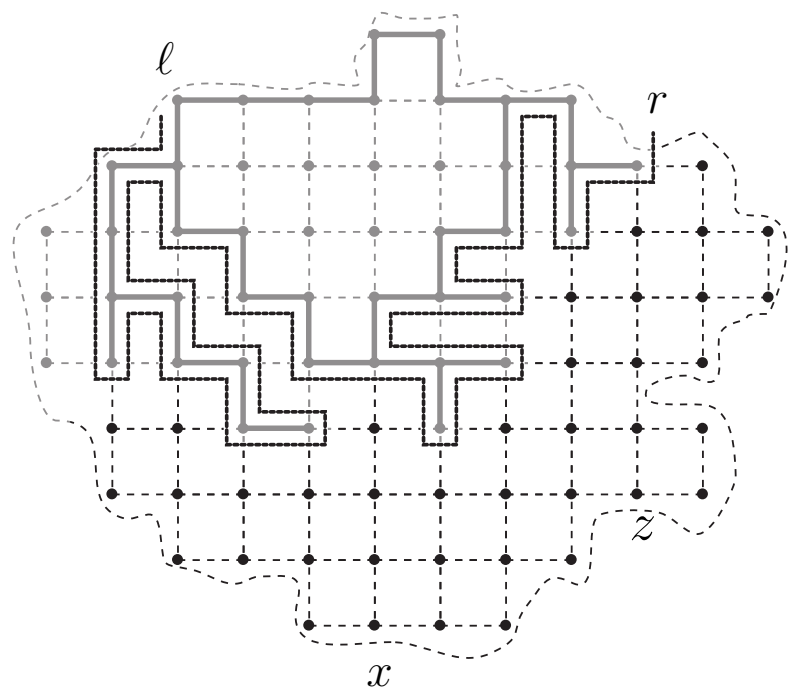

FiguRE 8.3. Expectation $\mathrm{E}_{\delta}^{\mathrm{A}}$ : the FK boundary conditions knowing the interface $\lambda_{\delta}$.

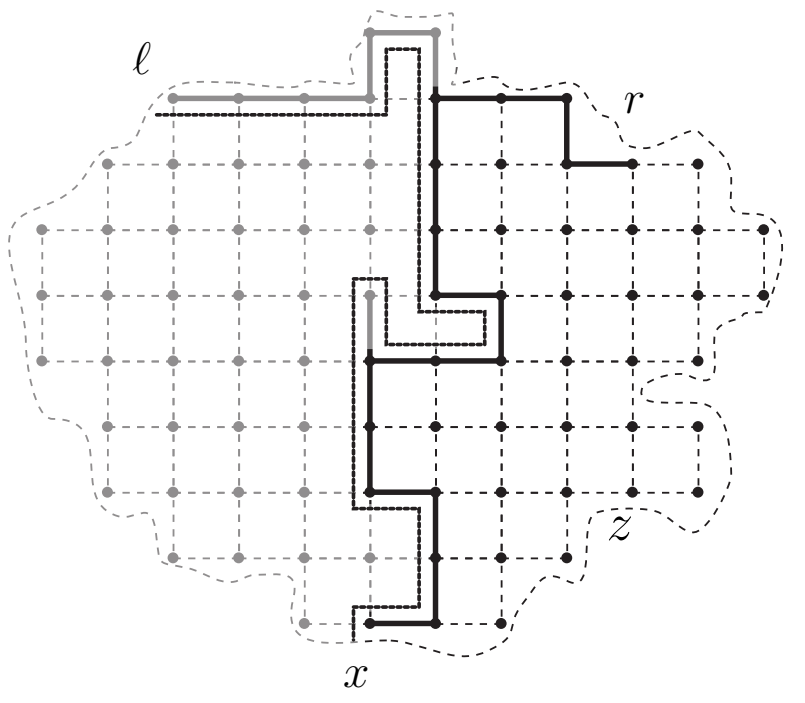

FiguRE 8.4. Expectation $\mathrm{E}_{\delta}^{\mathrm{B}}$ : the FK boundary conditions knowing the interface $\tilde{\lambda}_{\delta}$.

where $\mathbb{E}_{\delta}^{\mathrm{B}}$ is as in the statement of the proposition.

The proposition directly follows from Equations 8.1, 8.2, 8.4 (and Lemma 21 for the denominator) and the representation of $\Phi_{\delta}$ given by Proposition 11

$$
\Phi_{\delta}\left(\Omega_{\delta}, r, \ell, x, z\right)=\frac{\mathbb{E}_{\Omega_{\delta}}^{[r, \ell]_{+}}\left[\sigma_{x} \sigma_{z}\right]}{\mathbb{E}_{\Omega_{\delta}}^{[r, \ell]_{+}}\left[\sigma_{x}\right]} .
$$


8.6. Law of the conditioned FK interface. Let us finish this section by the following characterization of the conditioned FK interface, which will be useful in Section 10 to pass to the scaling limit.

Lemma 24. Let $\left(\Omega_{\delta}, r, \ell, x\right)$ be a discrete domain, $\lambda_{\delta}$ have the law of a critical FKIsing interface from $\ell$ to $r$ and let $\tilde{\lambda}_{\delta}$ have the law of a critical FK-Ising interface from $\ell$ to $r$, conditioned to pass at $x$, stopped as it hits $x$. For any $\epsilon>\delta$, and let $\tau_{\delta}^{\epsilon} \in \mathbb{N} \cup\{\infty\}$ be the first time that $\lambda_{\delta}$ hits $\mathbf{D}_{\Omega_{\delta}}(x, \epsilon)$, the connected component of $\left\{z \in \Omega_{\delta}:|z-x| \leq \epsilon\right\}$ containing $x$, and let $\tilde{\tau}_{\delta}^{\epsilon} \in \mathbb{N}$ be the first time that $\tilde{\lambda}_{\delta}$ hits $\mathbf{D}_{\Omega_{\delta}}(x, \epsilon)$. Let $\mathbb{P}_{\delta}^{\epsilon}$ and $\tilde{\mathbb{P}}_{\delta}^{\epsilon}$ denote the laws of $\lambda_{\delta}\left[0, \tau_{\delta}^{\epsilon}\right]$ and $\tilde{\lambda}_{\delta}\left[0, \tilde{\tau}_{\delta}^{\epsilon}\right]$. Then we have

$$
\operatorname{Supp}\left(\tilde{\mathbb{P}}_{\delta}^{\epsilon}\right)=\left\{\mu \in \operatorname{Supp}\left(\mathbb{P}_{\delta}^{\epsilon}\right): \mu \text { hits } \mathbf{D}_{\Omega_{\delta}}(x, \epsilon) \text { in finite time and } \mu \cap[x, r]=\emptyset\right\}
$$

and for any $\mu_{\delta}[0, n] \in \operatorname{Supp}\left(\tilde{\mathbb{P}}_{\delta}^{\epsilon}\right)$, we have the following expression for the RadonNikodym derivative:

$$
\frac{\mathrm{d} \tilde{\mathbb{P}}_{\delta}^{\epsilon}}{\mathrm{d} \mathbb{P}_{\delta}^{\epsilon}}\left(\mu_{\delta}[0, n]\right)=\frac{\mathbb{E}_{\Omega_{\delta} \backslash \mu_{\delta}}^{\left[r, \mu_{\delta}(n)\right]_{+}}\left[\sigma_{x}\right]}{\mathbb{E}_{\Omega_{\delta}}^{[r, \ell]_{+}}\left[\sigma_{x}\right]} .
$$

Proof. The first part of the statement is obvious. Using the domain Markov property and Doob's transform, for any $\mu_{\delta}[0, n] \in \operatorname{Supp}\left(\tilde{\mathbb{P}}_{\delta}^{\epsilon}\right)$ we obtain

$$
\frac{\mathrm{d} \tilde{\mathbb{P}}_{\delta}^{\epsilon}}{\mathrm{d} \mathbb{P}_{\delta}^{\epsilon}}\left(\mu_{\delta}[0, n]\right)=\frac{\mathbb{P}_{\Omega_{\delta} \backslash \mu_{\delta}}^{\left[r, \mu_{\delta}(n)\right]_{\mathrm{w}}}\left\{\lambda_{\delta}^{\dagger} \text { passes at } x\right\}}{\mathbb{P}_{\Omega_{\delta}}^{[r, \ell]_{\mathrm{w}}}\left\{\lambda_{\delta} \text { passes at } x\right\}} .
$$

where $\lambda_{\delta}^{\dagger}$ is a critical FK interface in $\Omega_{\delta} \backslash \mu_{\delta}[0, n]$ from $\mu_{\delta}(n)$ to $r$ (with wired boundary condition on $\left[r, \mu_{\delta}(n)\right]$ and free boundary condition on $\left.\left[\mu_{\delta}(n), r\right]\right)$.

By Lemmas 20 and 21, we get

$$
\frac{\mathbb{P}_{\Omega_{\delta} \backslash \mu_{\delta}}^{\left[r, \mu_{\delta}(n)\right]_{\mathrm{w}}}\left\{\lambda_{\delta}^{\dagger} \text { passes at } x\right\}}{\mathbb{P}_{\Omega_{\delta}}^{[r, \ell]_{\mathrm{w}}}\left\{\lambda_{\delta} \text { passes at } x\right\}}=\frac{\mathbb{E}_{\Omega_{\delta} \backslash \mu_{\delta}}^{\left[r, \mu_{\delta}(n)\right]_{+}}\left[\sigma_{x}\right]}{\mathbb{E}_{\Omega_{\delta}}^{[r, \ell]_{+}}\left[\sigma_{x}\right]} .
$$

Hence the result follows.

\section{Scaling limit of elementary correlations}

In this section, we discuss the scaling limit of the correlation functions appearing in Proposition 12 .

- The boundary spin-spin correlation with free boundary conditions: it is the numerator of the integrand in the expectation $\mathrm{E}_{\mathrm{A}}^{\delta}$.

- The boundary magnetization with mixed + and free boundary conditions: it is the integrand in the expectation $\mathrm{E}_{\mathrm{B}}^{\delta}$

The continuous counterparts of these quantities, the CFT correlation functions $\left\langle\sigma_{x} \sigma_{z}\right\rangle_{\Omega}^{\text {free }}$ and $\left\langle\sigma_{x}\right\rangle_{\Omega}^{[r, \ell]_{+}}$, are given in Definition 13 .

The convergence of the discrete correlation functions to the continuous ones is obtained by using discrete complex analysis techniques. The first correlation function appears in [Hon10a] and is closely related to the observable used in [ChSm09] 
to prove the convergence of the critical Ising interfaces to chordal SLE(3). The second correlation function is directly derived from the observable used in [ChSm11] to prove the convergence of the critical FK-Ising interfaces to chordal SLE(16/3).

Boundary correlation functions are very sensitive to the local geometry of the boundary: they both depend on the geometry of the limiting continuous domain (as they depend on the derivatives of the conformal mappings on the boundary), and of the way the domain is discretized. These issues are important, since we need to prove the convergence of the observable $\Phi_{\delta}$ in domains that can a priori be very rough, since they are slitted by an interface, which tends to a continuous fractal. The point is that the observable $\Phi_{\delta}$ is a ratio of two correlation functions: the dependences of the numerator and the denominator on the fine geometry of the continuous domain and its discretization compensate each other, and this allows to obtain the desired result.

Another related issue is the uniformity of the convergence which is needed for the proof of Proposition 14 the convergence should be uniform with respect to the shape and the discretization of the domain: indeed, in the end, we want to be able to say that for $\delta$ small enough, the discrete observable $\Phi_{\delta}$ is close to its continuous counterpart $\Phi$, uniformly over all the possible realizations of the dipolar interface $\lambda_{\delta}$.

The discrete complex analysis details required to prove these results are presented in Section 13 .

9.1. Two-point function. The scaling limit of the boundary spin correlations on discrete vertex domains with free boundary conditions (see Section 2.2) is given by the following theorem.

Theorem 25. Let $(\Omega, x, z)$ be a domain such that $x$ and $z$ lie on vertical parts of $\partial \Omega$ and let $\left(\Omega_{\delta}, x_{\delta}, z_{\delta}\right)_{\delta>0}$ be a family of discrete vertex domains approximating $(\Omega, x, z)$. Then we have

$$
\frac{1}{\delta} \mathbb{E}_{\Omega_{\delta}}^{\text {free }}\left[\sigma_{x_{\delta}} \sigma_{z_{\delta}}\right] \underset{\delta \rightarrow 0}{\longrightarrow}\left\langle\sigma_{x} \sigma_{z}\right\rangle_{\Omega}^{\text {free }}
$$

where $\left\langle\sigma_{x} \sigma_{z}\right\rangle_{\Omega}^{\text {free }}$ is as in Definition 13 .

This result is derived in ([Hon10a, , Theorem 1), when the discretization $\Omega_{\delta}$ is the largest connected component of $\Omega \cap \delta \mathbb{Z}^{2}$ and when $\Omega$ is assumed $\mathcal{C}^{1}$. The question of the convergence for more general domains is discussed in Section 9.3 . The article ChSm09] gives (using the Kramers-Wannier duality) the convergence of ratios of such spin correlations at different locations on the boundary. The non-universal constant $\frac{1}{\pi}$ appearing in Definition 13 is however not obtained there, while it is important for our purposes.

Remark 26. If $x$ and $z$ lie on a smooth part of $\Omega$, it is easy to check that $\left\langle\sigma_{x} \sigma_{z}\right\rangle_{\Omega}^{\text {free }}$ is equal (up to a constant factor) to $\sqrt{E_{\Omega}(x, z)}$, where $E$ is the excursion Poisson kernel defined by

$$
E_{\Omega}(x, z):=\frac{\partial}{\partial \nu_{\text {in }}(x)} P_{\Omega}(z, \cdot)=\frac{\partial}{\partial \nu_{\text {in }}(z)} P_{\Omega}(x, \cdot),
$$

where $P_{\Omega}(\cdot, \cdot): \partial \Omega \times \Omega \rightarrow \mathbb{R}$ is the Poisson kernel and $\frac{\partial}{\partial \nu_{\text {in }}(x)}$ denotes the inward normal derivative at $x$. From the monotonicity properties of the Poisson kernel with 
respect to domain, we easily get that if $\Upsilon \subset \Omega$ are two domains coinciding in smooth neighborhoods of $x$ and $z$, we have

$$
\left\langle\sigma_{x} \sigma_{z}\right\rangle_{\Upsilon}^{\text {free }} \leq\left\langle\sigma_{x} \sigma_{z}\right\rangle_{\Omega}^{\text {free }}
$$

\subsection{One-point function.}

Theorem 27. Let $(\Omega, r, \ell, x)$ be a domain such that such that $x$ lies on a vertical part of $\partial \Omega$ and let $\left(\Omega_{\delta}, r_{\delta}, \ell_{\delta}, x_{\delta}\right)_{\delta>0}$ be a family of discrete vertex domains approximating $(\Omega, r, \ell, x)$. Then we have

$$
\frac{1}{\sqrt{\delta}} \mathbb{E}_{\Omega_{\delta}}^{\left[r_{\delta}, \ell_{\delta}\right]_{+}}\left[\sigma_{x_{\delta}}\right] \underset{\delta \rightarrow 0}{\longrightarrow}\left\langle\sigma_{x}\right\rangle_{\Omega}^{[r, \ell]_{+}}
$$

where $\left\langle\sigma_{x}\right\rangle_{\Omega}^{[r, \ell]_{+}}$is as in Definition 13 .

This result follows essentially from ([Smi10a], Remark 2.4): using the FK interpretation of the boundary magnetization of Lemmas 20 and 21 , we obtain that

$$
\mathbb{E}_{\Omega_{\delta}}^{\left[r_{\delta}, \ell_{\delta}\right]_{+}}\left[\sigma_{x_{\delta}}\right]=\mathbb{P}_{\Omega_{\delta}}^{\left[r_{\delta}, \ell_{\delta}\right]_{\mathrm{w}}}\left[\gamma_{\delta} \text { passes at } x_{\delta}\right]
$$

where $\gamma_{\delta}$ is the FK interface defined in the previous section. The right-hand side is the absolute value of Smirnov's observable, whose convergence is the main theorem (Theorem 2.2) of [Smi10a]. That article proves the convergence in the bulk, but as explained in [ChSm09], it is not too difficult to extend the convergence to the straight parts of the boundary (see [Hon10a] for a version of this result specialized to the square lattice).

Notice that it is again important for our purposes to obtain the correct latticedependent constant $\sqrt{\frac{\sqrt{2}+1}{2 \pi}}$ appearing in Definition 13 ; it is quite easy to derive using the lattice construction detailed in [Smi10a].

Remark 28. Notice that, if $x$ lies on a smooth part of $\partial \Omega$, it is easy to check that $\left\langle\sigma_{x}\right\rangle_{\Omega}^{[r, \ell]_{+}}$is equal to a multiple of

$$
\sqrt{\frac{\partial}{\partial \nu_{\text {in }}(x)} \mathbf{H}_{\Omega}(\cdot,[r, \ell])},
$$

where $\frac{\partial}{\partial \nu_{\operatorname{in}(x)}}$ is the inner normal derivative at $x$ and $\mathbf{H}_{\Omega}(z,[r, \ell])$ is the harmonic measure of the arc $[r, \ell]$ in $\Omega$, viewed from $z$ (the probability that a $2 \mathrm{D}$ Brownian motion starting at $z$ exits $\Omega$ on $[r, \ell])$.

9.3. Rough boundaries and uniformity. A significant technical difficulty is to extend the convergence results of Theorems 25 and 27 to a setup that we can use to prove the convergence of the observable $\Phi_{\delta}$. These convergence results will most of the time not hold true for more general boundaries.

However, the convergence of the ratio of such correlation functions will be true if the same points on the same rough parts of the boundary appear both in the numerator and the denominator, even if the domains that are considered are different, or even if one divides a two-point function by a one-point function.

Let us state the two particular cases of this phenomenon that we will need. Notice that the right-hand sides are well-defined for any $x$, since we can use the same local conformal charts to look at the derivatives of the conformal maps involved in the numerator and the denominator. 
Theorem 29. Let $(\Theta, y, t, x),(\tilde{\Theta}, \tilde{y}, \tilde{t}, x)$ and $(\Xi, x, s)$ be domains which coincide in a neighborhood of the boundary point $x$. Suppose that s lies on a vertical part $\mathfrak{v}$ of $\partial \Xi$. Let $\left(\Theta_{\delta}, y_{\delta}, t_{\delta}, x_{\delta}\right),\left(\tilde{\Theta}_{\delta}, \tilde{y}_{\delta}, \tilde{t}_{\delta}, x_{\delta}\right)$ and $\left(\Xi_{\delta}, x_{\delta}, s_{\delta}\right)$ be discretizations of these domains coinciding in a neighborhood of $x_{\delta}$, converging to their continuous counterparts in the sense of the metric of Section 2.6. Suppose that for each $\delta>0, \partial \Xi_{\delta}$ contains a vertical part $\mathfrak{v}_{\delta}$ around $s_{\delta}$ and that as $\delta \rightarrow 0, \mathfrak{v}_{\delta}$ converges to $\mathfrak{v}$. Then we have

$$
\begin{aligned}
\frac{1}{\sqrt{\delta}} \frac{\mathbb{E}_{\Xi_{\delta}}^{\text {free }}\left[\sigma_{x_{\delta}} \sigma_{s_{\delta}}\right]}{\mathbb{E}_{\Theta_{\delta}}^{\left[y_{\delta}, t_{\delta}\right]_{+}}\left[\sigma_{x_{\delta}}\right]} \underset{\delta \rightarrow 0}{\longrightarrow} \frac{\left\langle\sigma_{x} \sigma_{s}\right\rangle_{\Xi}^{\text {free }}}{\left\langle\sigma_{x}\right\rangle_{\Theta}^{[y, t]_{+}}} \\
\frac{\mathbb{E}_{\tilde{\Theta}_{\delta}}^{\left[\tilde{y}_{\delta}, \tilde{t}_{\delta}\right]_{+}}\left[\sigma_{x_{\delta}}\right]}{\mathbb{E}_{\Theta_{\delta}}^{\left[y_{\delta}, t_{\delta}\right]_{+}}\left[\sigma_{x_{\delta}}\right]} \underset{\delta \rightarrow 0}{\longrightarrow} \frac{\left\langle\sigma_{x}\right\rangle_{\tilde{\Theta}}^{\left[\tilde{y}_{\delta}, \tilde{t}_{\delta}\right]_{+}}}{\left\langle\sigma_{x}\right\rangle_{\Theta}^{\left[y_{\delta}, t_{\delta}\right]_{+}}}
\end{aligned}
$$

The convergence is locally uniform with respect to the domains.

To prove this result, the main idea is to cut the domains near $x$, obtaining a domain $(\Upsilon, p, q, x)$ such that $\Upsilon \subset \Theta \cap \tilde{\Theta} \cap \Xi,[p, q]$ is made of straight segments and $[q, p] \subset \partial \Theta \cap \partial \tilde{\Theta} \cap \partial \Xi$. The discrete correlation functions of the left-hand sides come from discrete holomorphic observables, and in particular are the boundary values of discrete holomorphic functions, with the same type of boundary values on (the discretizations of) $[q, p]$.

It turns out that we can use this fact to represent the discrete holomorphic functions involved as convolution of their boundary values on $[p, q]$ (which are wellcontrolled, since they are not on the boundaries of the original domains) with the discrete holomorphic observable of [ChSm09]. The convergence of the ratios of this latter observable is addressed by Chelkak and Smirnov [ChSm09], and hence we can use their result to obtain ours.

The details are presented in Section 13 .

\section{SLE variants and scaling limit of FK interfaces}

In the previous section, we discussed the convergence of the elementary correlation functions. The other main ingredient to prove Proposition 14 is the convergence of the FK interfaces to variants of $\operatorname{SLE}(16 / 3)$. Let us recall that there are two interfaces that are considered, both in the discrete domain $\left(\Omega_{\delta}, r_{\delta}, \ell_{\delta}, x_{\delta}, z_{\delta}\right)$.

- The interface $\lambda_{\delta}$, which has the law of an FK interface from $r_{\delta}$ to $\ell_{\delta}$.

- The interface $\tilde{\lambda}_{\delta}$, which has the law of an FK interface from $r_{\delta}$ to $\ell_{\delta}$, conditioned to pass at $x_{\delta}$, stopped upon hitting $x_{\delta}$.

\subsection{Chordal $\operatorname{SLE}(\kappa)$ and $\operatorname{SLE}(\kappa ; \rho)$.}

10.1.1. Chordal $S L E(\kappa)$. We now recall the definitions of the simplest and most standard variant of SLE, which is chordal $\operatorname{SLE}(\kappa)$; it is most naturally defined on the upper half-plane $\mathbb{H}:=\{z \in \mathbb{C}: \Im \mathfrak{m}(z)>0\}$, in the same manner as dipolar $\operatorname{SLE}(\kappa)$ is most naturally defined on the strip. Chordal $\operatorname{SLE}(\kappa)$ in $\mathbb{H}$ from 0 (the 
source) to $\infty$ (the destination) is defined by the half-plane Loewner chain (the halfplane counterpart of the strip Loewner chain)

$$
\begin{aligned}
\partial_{t} g_{t}(z) & =\frac{2}{g_{t}(z)-U_{t}}, \\
g_{0}(z) & =z
\end{aligned}
$$

where the driving function $\left(U_{t}\right)_{t \geq 0}$ has the law of $\left(\sqrt{\kappa} B_{t}\right)_{t \geq 0}$, where $\left(B_{t}\right)_{t \geq 0}$ is a standard Brownian motion. It can be shown that for any time $t \geq 0, g_{t}$ is conformal map from $H_{t} \subset \mathbb{H}$ to $\mathbb{H}$, where $H_{t}$ is the unbounded component of $\mathbb{H} \backslash \lambda[0, t]$, where $\lambda$ is a curve from 0 to $\infty$, called the (chordal) $S L E$ trace, and that $g_{t}(\lambda(t))=U_{t}$. By (chordal) $S L E$, we mean the trace $\lambda$ (as an oriented unparametrized random curve).

For $\kappa \in[0,4], \operatorname{SLE}(\kappa)$ is almost surely a simple curve, for $\kappa \in(4,8)$, it has almost surely double points (but it does not cross itself) and for $\kappa \geq 8$, it is almost surely space-filling [Law05, RoSc05].

In an arbitrary simply connected domain $(\Omega, a, b), \operatorname{SLE}(\kappa)$ is defined as the conformal image of chordal SLE in $(\mathbb{H}, 0, \infty)$ by a conformal mapping $\varphi:(\mathbb{H}, 0, \infty) \rightarrow$ $(\Omega, a, b)$. Almost sure continuity of the $\operatorname{SLE}(\kappa)$ trace in arbitrary domains is shown in GRS08.

10.1.2. $\operatorname{SLE}(\kappa ; \rho)$. A very useful variant of $\operatorname{SLE}(\kappa)$ is $\operatorname{SLE}(\kappa ; \rho)$, which is a process defined in a domain with three marked boundary points. It is defined in $(\mathbb{H}, 0, x, \infty)$, where $x \in \mathbb{R} \backslash\{0\}$, by a half-plane Loewner chain $\left(\tilde{g}_{t}\right)_{t \geq 0}$ with driving force $\left(\tilde{U}_{t}\right)_{t \geq 0}$, which is defined by the following Itô stochastic differential equation

$$
\begin{aligned}
\mathrm{d} \tilde{U}_{t} & =\sqrt{\kappa} \mathrm{d} B_{t}+\frac{\rho \mathrm{d} t}{\tilde{U}_{t}-O_{t}} \\
\mathrm{~d} O_{t} & =\frac{2 \mathrm{~d} t}{O_{t}-\tilde{U}_{t}} \\
O_{0} & =x .
\end{aligned}
$$

The process is defined up to the the first time $\tau$ when $\tilde{U}_{t}=O_{t}$. As before it can be shown that, for each $0<t<\tau, \tilde{g}_{t}$ is a conformal map $H_{t} \rightarrow \mathbb{H}$, where $H_{t}$ is the unbounded connected component of $\mathbb{H} \backslash \tilde{\lambda}[0, t]$, where $\tilde{\lambda}$ is a curve, called the $\operatorname{SLE}(\kappa ; \rho)$ trace. The $\operatorname{SLE}(\kappa ; \rho)$ trace emanates at 0 and is well-defined up to the first time when it disconnects $x$ from $\infty$.

By Girsanov's theorem, we have that the initial segments of the $\operatorname{SLE}(\kappa ; \rho)$ trace, before the time when $x$ and $\infty$ get disconnected by the trace, are absolutely continuous with respect to the initial segments of chordal $\operatorname{SLE}(\kappa)$ in $(\mathbb{H}, 0, \infty)$, see Lemma 95 in Appendix B. We will simply refer to $\operatorname{SLE}(\kappa ; \rho)$ trace as $\operatorname{SLE}(\kappa ; \rho)$.

In an arbitrary domain $(\Omega, a, b, c), \operatorname{SLE}(\kappa ; \rho)$ from $a$ (the source) to $c$ (the observation point) with force point $b$ is defined as the image of $\operatorname{SLE}(\kappa ; \rho)$ in $(\mathbb{H}, 0, x, \infty)$ by the conformal mapping $\phi:(\mathbb{H}, 0, x, \infty) \rightarrow(\Omega, a, b, c)$.

10.1.3. Useful variants. The following variants of $\operatorname{SLE}(\kappa ; \rho)$ (on $(\mathbb{H}, 0, x, \infty)$ for definiteness) are relevant in this paper:

- $\operatorname{SLE}(\kappa ; \kappa-6)$ : for $\kappa \geq 0$, it has the same law as chordal $\operatorname{SLE}(\kappa)$ in $(\mathbb{H}, 0, x)$, until the first time $x$ gets disconnected from $\infty$ by $\tilde{\gamma}$ (see [ScWi05, Theorem $13])$. 
- $\operatorname{SLE}(\kappa ; \kappa-8)$ : has the law of a chordal $\operatorname{SLE}(\kappa)$, conditioned to hit the point $x$ (more precisely: to hit a ball of radius $\epsilon$ around $x$, as $\epsilon \rightarrow 0$ ). As we will only be interested in the $\kappa=16 / 3, \rho=-8 / 3$ case (see Theorem 32), we will not make use of this general result (note that it is used in [BeIz10] for the $\kappa=6$ case).

- $\operatorname{SLE}\left(\kappa ; \frac{\kappa-6}{2}\right)$ : has the law of dipolar SLE in $(\mathbb{H}, 0, x, \infty)$ Kyt06a, ScWi05. Although we do not make use of this fact, it is worth mentioning: in view of Lemma 95, it tells us in particular that chordal and dipolar SLE(3) are absolutely continuous with respect to each other.

The following lemma provides a useful characterization of SLE $(\kappa ; \kappa-8)$, in the case when $\kappa=16 / 3$. It is a continuous version of Lemma 24. Let us use, for definiteness, the time parametrization of the SLE trace inherited from the half-plane Loewner chain via the conformal map $\varphi:(\mathbb{H}, 0,1, \infty) \rightarrow(\Omega, r, \ell, x)$. Denote by $\mathbf{D}_{\Omega}(x, \epsilon)$ the connected component of $\{z \in \bar{\Omega}:|z-x| \leq \epsilon\}$ containing $x$.

Lemma 30. Consider the domain $(\Omega, r, \ell, x)$. Let $\lambda$ have the law of an SLE(16/3) curve in $\Omega$ from $\ell$ to $r$ and let $\tilde{\lambda}$ have the law of an $S L E(16 / 3 ;-8 / 3)$ curve in $\Omega$ with starting point $\ell$, observation point $r$ and force point $x$.

For $\epsilon>0$, let $\tau^{\epsilon}$ be the (possibly infinite) first time that $\lambda$ hits $\mathbf{D}_{\Omega}(x, \epsilon)$, and let $\tilde{\tau}^{\epsilon}$ be the first time that $\tilde{\lambda}$ hits $\mathbf{D}_{\Omega}(x, \epsilon)$. Let $\mathbb{P}^{\epsilon}$ be the law of $\lambda\left[0, \tau^{\epsilon}\right]$ and $\tilde{\mathbb{P}}^{\epsilon}$ be the law of $\tilde{\lambda}\left[0, \tilde{\tau}^{\epsilon}\right]$. Then we have

$$
\operatorname{Supp}\left(\tilde{\mathbb{P}}^{\epsilon}\right)=\left\{\mu \in \operatorname{Supp}\left(\mathbb{P}^{\epsilon}\right): \mu \text { hits } \mathbf{D}_{\Omega}(x, \epsilon) \text { in finite time and } \mu \cap[x, r]=\emptyset\right\}
$$

and for any curve $\mu[0, t] \in \operatorname{Supp}\left(\tilde{\mathbb{P}}^{\epsilon}\right)$ we have the following expression for the Radon-Nikodym derivative:

$$
\frac{\mathrm{d} \tilde{\mathbb{P}}^{\epsilon}}{\mathrm{d} \mathbb{P}^{\epsilon}}(\mu[0, t])=\frac{\left\langle\sigma_{x}\right\rangle_{\Omega \backslash \mu[0, t]}^{[r, \mu(t)]_{+}}}{\left\langle\sigma_{x}\right\rangle_{\Omega}^{[r, \ell]_{+}}} .
$$

The proof is given in Appendix B.

10.2. Convergence of the FK interfaces. The convergence of $\gamma_{\delta}$ is a celebrated theorem of Smirnov [Smi06].

Theorem 31 ([Smi06]). If $(\Omega, r, \ell)$ is a domain and $\left(\Omega_{\delta}, r_{\delta}, \ell_{\delta}\right)_{\delta}$ is a vertex domain discretization of it, the critical FK-Ising interface $\gamma_{\delta}$ from $r_{\delta}$ to $\ell_{\delta}$ converges in law to $\gamma$ as $\delta \rightarrow 0$, where $\gamma$ is a chordal SLE(16/3) trace in $\Omega$ from $r$ to $\ell$. The convergence is locally uniform with respect to the domains.

The convergence of the FK interface from $r_{\delta}$ to $\ell_{\delta}$, conditioned to pass through the point $x_{\delta}$, can then be derived from Theorem 31 .

Theorem 32. Let $(\Omega, r, \ell, x)$ be a domain and let $\left(\Omega_{\delta}, r_{\delta}, \ell_{\delta}, x_{\delta}\right)$ be a vertex domain discretization of it, and let $\tilde{\lambda}_{\delta}$ have the law of a critical FK-Ising interface in $\Omega_{\delta}$ from $\ell_{\delta}$ to $r_{\delta}$, conditioned to pass at $x_{\delta}$, stopped as it hits $x_{\delta}$. Then $\tilde{\lambda}_{\delta}$ converges in law to $\tilde{\lambda}$ as $\delta \rightarrow 0$, where $\tilde{\lambda}$ is an $S L E(16 / 3 ;-8 / 3)$ trace in $(\Omega, r, \ell, x)$, with source $r$, force point $x$ and observation point $\ell$. The convergence is locally uniform with respect to the domains $(\Omega, r, \ell, x)$. 
Proof. Let $\lambda_{\delta}$ have the law of a critical FK-Ising interface in $\Omega_{\delta}$ from $\ell_{\delta}$ to $r_{\delta}$ and $\lambda$ have the law of an $\operatorname{SLE}(16 / 3)$ in $\Omega$ from $\ell$ to $r$. For $\epsilon>0$ and for $\delta$ small enough, let $\mathbf{D}_{\Omega_{\delta}}\left(x_{\delta}, \epsilon\right)$ be the connected component of $\Omega_{\delta} \cap\{z \in \mathbb{C}:|z-x| \leq \epsilon\}$ containing $x_{\delta}$, let $\tau_{\delta}^{\epsilon}$ be the first time that $\lambda_{\delta}$ hits $\mathbf{D}_{\Omega_{\delta}}\left(x_{\delta}, \epsilon\right)$ and let $\tilde{\tau}_{\delta}^{\epsilon}$ be the first time that $\tilde{\lambda}_{\delta}$ hits $\mathbf{D}_{\Omega_{\delta}}\left(x_{\delta}, \epsilon\right)$; denote by $\tau^{\epsilon}$ and $\tilde{\tau}^{\epsilon}$ the corresponding stopping times for $\lambda$ and $\tilde{\lambda}$, as in Lemma 30 . Let $\mathbb{P}_{\delta}^{\epsilon}, \tilde{\mathbb{P}}_{\delta}^{\epsilon}, \mathbb{P}^{\epsilon}$ and $\tilde{\mathbb{P}}^{\epsilon}$ denote the respective laws of $\lambda_{\delta}\left[0, \tau_{\delta}^{\epsilon}\right]$, $\tilde{\lambda}_{\delta}\left[0, \tilde{\tau}_{\delta}^{\epsilon}\right], \lambda\left[0, \tau^{\epsilon}\right]$ and $\tilde{\lambda}\left[0, \tilde{\tau}^{\epsilon}\right]$. We can now put together the following four results:

- Let $\mu_{\delta}[0, n] \in \operatorname{Supp}\left(\tilde{\mathbb{P}}_{\delta}^{\epsilon}\right)$. By Lemma 24 , we have

$$
\frac{\mathrm{d} \tilde{\mathbb{P}}_{\delta}^{\epsilon}}{\mathrm{d} \mathbb{P}_{\delta}^{\epsilon}}\left(\mu_{\delta}[0, n]\right)=\frac{\mathbb{E}_{\Omega_{\delta} \backslash \mu_{\delta}}^{\left[r_{\delta}, \mu_{\delta}(n)\right]_{+}}\left[\sigma_{x_{\delta}}\right]}{\mathbb{E}_{\Omega_{\delta}}^{\left[r_{\delta}, \ell_{\delta}\right]_{+}}\left[\sigma_{x_{\delta}}\right]} .
$$

- By Theorem 29, if $\mu_{\delta}\left[0, n_{\delta}\right] \rightarrow \mu[0, t] \in \operatorname{Supp}\left(\tilde{\mathbb{P}}^{\epsilon}\right)$, we get

$$
\frac{\mathbb{E}_{\Omega_{\delta} \backslash \mu_{\delta}}^{\left[r_{\delta}, \mu_{\delta}(n)\right]_{+}}\left[\sigma_{x_{\delta}}\right]}{\mathbb{E}_{\Omega_{\delta}}^{\left[r_{\delta}, \ell_{\delta}\right]_{+}}\left[\sigma_{x_{\delta}}\right]} \underset{\delta \rightarrow 0}{\longrightarrow} \frac{\left\langle\sigma_{x}\right\rangle_{\Omega \backslash \mu[0, t]}^{[r, \mu(t)]_{+}}}{\left\langle\sigma_{x}\right\rangle_{\Omega}^{[r, \ell]_{+}}}
$$

locally uniformly with respect to $\mu$ and $(\Omega, r, \ell, x)$.

- From Theorem 31 and standard arguments, we get

$$
\mathbb{P}_{\delta}^{\epsilon} \underset{\delta \rightarrow 0}{\longrightarrow} \mathbb{P}^{\epsilon},
$$

locally uniformly with respect to $(\Omega, r, \ell, x)$.

- By Lemma 30, for any $\mu[0, t] \in \operatorname{Supp}\left(\tilde{\mathbb{P}}^{\epsilon}\right)$, we have

$$
\frac{\mathrm{d} \tilde{\mathbb{P}}^{\epsilon}}{\mathrm{d} \mathbb{P}^{\epsilon}}(\mu[0, t])=\frac{\left\langle\sigma_{x}\right\rangle_{\Omega \backslash \mu[0, t]}^{[r, \mu(t)]_{+}}}{\left\langle\sigma_{x}\right\rangle_{\Omega}^{[r, \ell]_{+}}} .
$$

From these four results, we easily deduce that

$$
\tilde{\mathbb{P}}_{\delta}^{\epsilon} \underset{\delta \rightarrow 0}{\longrightarrow} \tilde{\mathbb{P}}^{\epsilon},
$$

locally uniformly with respect to $(\Omega, r, \ell, x)$. Finally, thanks to Lemma 33 below, we can pass to the $\epsilon \rightarrow 0$ limit and complete the proof of the theorem: we get that for small $\epsilon>0$, with uniformly high probability, $\tilde{\lambda}_{\delta}$ will always remain close to $x_{\delta}$ after time $\tau_{\delta}^{\epsilon}$, and similarly that $\tilde{\lambda}$ will remain close to $x$ after time $\tau^{\epsilon}$.

Lemma 33. For any $R>0$, the probability that $\tilde{\lambda}_{\delta}$ exits $\mathbf{D}_{\Omega_{\delta}}\left(x_{\delta}, R\right)$ after the time $\tilde{\tau}_{\delta}^{\epsilon}$ tends to 0 as $\epsilon \rightarrow 0$, uniformly with respect to $\left(\Omega_{\delta}, r_{\delta}, \ell_{\delta}, x_{\delta}\right)$. Similarly, for any $\rho>0$, the probability that $\tilde{\lambda}$ exits $\mathbf{D}_{\Omega}(x, \rho)$ after the time $\tilde{\tau}^{\epsilon}$ tends to 0 as $\epsilon \rightarrow 0$, uniformly with respect to $\left(\Omega_{\delta}, r_{\delta}, \ell_{\delta}, x_{\delta}\right)$

The proof is given in Appendix C.

\section{From FK expectations to SLE expectations}

In this section, we put together the results of the two previous sections to obtain Proposition 14, which is the convergence of the FK-Ising expectations $\mathrm{E}_{\delta}^{\mathrm{A}}$ and $\mathrm{E}_{\delta}^{\mathrm{B}}$ 
to SLE expectations. Recall that $\mathrm{E}_{\delta}^{\mathrm{A}}$ and $\mathrm{E}_{\delta}^{\mathrm{B}}$ are defined by

$$
\begin{aligned}
& \mathrm{E}_{\delta}^{\mathrm{A}}\left(\Omega_{\delta}, r_{\delta}, \ell_{\delta}, x_{\delta}, z_{\delta}\right):=\mathbb{E}_{\delta}^{\mathrm{A}}\left[\frac{\mathbb{E}_{\Omega_{\delta} \backslash \lambda_{\delta}}^{\text {free }}\left[\sigma_{x_{\delta}} \sigma_{z_{\delta}}\right]}{\mathbb{E}_{\Omega_{\delta}}^{\left[r_{\delta}, \ell_{\delta}\right]_{+}}\left[\sigma_{x_{\delta}}\right]}\right], \\
& \mathrm{E}_{\delta}^{\mathrm{B}}\left(\Omega_{\delta}, r_{\delta}, \ell_{\delta}, x_{\delta}, z_{\delta}\right):=\mathbb{E}_{\delta}^{\mathrm{B}}\left[\mathbb{E}_{\Omega_{\delta} \backslash \tilde{\lambda}_{\delta}}^{\left[r_{\delta}, x_{\delta}\right]_{+}}\left[\sigma_{z_{\delta}}\right]\right]
\end{aligned}
$$

where $\gamma_{\delta}$ has the law of an FK interface from $r_{\delta}$ to $\ell_{\delta}$, and $\tilde{\lambda}_{\delta}$ has the law of $\lambda_{\delta}$ conditioned to pass at $x_{\delta}$ and stopped when it hits $x_{\delta}$.

Proposition (Proposition 14). As $\delta \rightarrow 0$ and $\left(\Omega_{\delta}, r_{\delta}, \ell_{\delta}, x_{\delta}, z_{\delta}\right) \rightarrow(\Omega, r, \ell, x, z)$, we have

$$
\begin{aligned}
& \frac{1}{\sqrt{\delta}} \mathrm{E}_{\delta}^{\mathrm{A}}\left(\Omega_{\delta}, r_{\delta}, \ell_{\delta}, x_{\delta}, z_{\delta}\right) \underset{\delta \rightarrow 0}{\longrightarrow} \mathrm{E}^{\mathrm{A}}(\Omega, r, \ell, x, z) \\
& \frac{1}{\sqrt{\delta}} \mathrm{E}_{\delta}^{\mathrm{B}}\left(\Omega_{\delta}, r_{\delta}, \ell_{\delta}, x_{\delta}, z_{\delta}\right) \underset{\delta \rightarrow 0}{\longrightarrow} \mathrm{E}^{\mathrm{B}}(\Omega, r, \ell, x, z),
\end{aligned}
$$

where the continuous expectations $\mathrm{E}^{\mathrm{A}}(\Omega, r, \ell, x, z)$ and $\mathrm{E}^{\mathrm{B}}(\Omega, r, \ell, x, z)$ are defined by

where

$$
\begin{aligned}
& \mathrm{E}^{\mathrm{A}}(\Omega, r, \ell, x, z):=\mathbb{E}^{\mathrm{A}}\left[\frac{\left\langle\sigma_{x} \sigma_{z}\right\rangle_{\Omega \backslash \lambda}^{\text {free }}}{\left\langle\sigma_{x}\right\rangle_{\Omega}^{[r, \ell]_{+}}}\right], \\
& \mathrm{E}^{\mathrm{B}}(\Omega, r, \ell, x, z):=\mathbb{E}^{\mathrm{B}}\left[\left\langle\sigma_{z}\right\rangle_{\Omega \backslash \tilde{\lambda}}^{[r, \ell]_{+}}\right],
\end{aligned}
$$

- the correlation functions are as in Definition 13 .

- the expectation $\mathbb{E}^{\mathrm{A}}$ is over the realizations $\lambda$ of a chordal $S L E(16 / 3)$ trace from $\ell$ to $r$.

- the expectation $\mathbb{E}^{\mathrm{B}}$ is over the realizations $\tilde{\lambda}$ of an $S L E(16 / 3 ;-8 / 3)$ trace starting from $\ell$, with observation point $r$ and force point $x$, stopped upon hitting $x$.

- the integrand in $\mathbb{E}^{\mathrm{A}}$ is defined as 0 if $x$ and $z$ are in different connected components of $\Omega \backslash \lambda$.

The convergence is locally uniform with respect to the domains.

The proof of the first part of the theorem (convergence of $\mathrm{E}_{\delta}^{\mathrm{A}}$ ) is given in Section 11.1 and the proof of the second part (convergence of $\mathrm{E}_{\delta}^{\mathrm{B}}$ ) is given in Section 11.2 .

\subsection{Proof of convergence of $\mathrm{E}_{\delta}^{\mathrm{A}}$.}

Proof of Proposition 14, part A. There are three ingredients (the types of convergence are as in Section 2.6):

- Convergence of the probability measure: from Theorem 31, we have that the discrete FK interface $\lambda_{\delta}$ converges to the chordal SLE(16/3) trace $\lambda$.

- Convergence of the integrand: from Theorem 29, for any fixed curves $\lambda_{\delta}^{*}$ converging to a $\lambda^{*}$ as $\delta \rightarrow 0$, we have

$$
\frac{1}{\sqrt{\delta}} \frac{\mathbb{E}_{\Omega_{\delta} \backslash \lambda_{\delta}^{*}}^{\text {free }}\left[\sigma_{x_{\delta}} \sigma_{z_{\delta}}\right]}{\mathbb{E}_{\Omega_{\delta}}^{\left[r_{\delta}, \ell_{\delta}\right]_{+}}\left[\sigma_{x_{\delta}}\right]} \rightarrow \frac{\left\langle\sigma_{x} \sigma_{z}\right\rangle_{\Omega \backslash \lambda^{*}}}{\left\langle\sigma_{x}\right\rangle_{\Omega}},
$$

where the convergence with respect to $\lambda_{\delta}^{*}$ and $\Omega$ is locally uniform. 
- The integrand is uniformly bounded: by monotonicity of spin correlations with free boundary conditions (which follows from FKG inequality, see [Gri06]), we have

$$
0 \leq \frac{1}{\sqrt{\delta}} \frac{\mathbb{E}_{\Omega_{\delta} \backslash \lambda_{\delta}}^{\text {free }}\left[\sigma_{x_{\delta}} \sigma_{z_{\delta}}\right]}{\mathbb{E}_{\Omega_{\delta}}^{\left[r_{\delta}, \ell_{\delta}\right]_{+}}\left[\sigma_{x_{\delta}}\right]} \leq \frac{1}{\sqrt{\delta}} \frac{\mathbb{E}_{\Omega_{\delta}}^{\text {free }}\left[\sigma_{x_{\delta}} \sigma_{z_{\delta}}\right]}{\mathbb{E}_{\Omega_{\delta}}^{\left[r_{\delta}, \ell_{\delta}\right]_{+}}\left[\sigma_{x_{\delta}}\right]} .
$$

As the right-hand side is uniformly convergent, the left-hand side is uniformly bounded.

We deduce the convergence as follows: by the first point, for any $\epsilon_{0}>0$, there exists a $\delta_{0}>0$ (locally uniform with respect to $\Omega$ ) such that for any $\delta \leq \delta_{0}$, we have

$$
\mathbb{P}\left\{\mathrm{d}_{\infty}\left(\lambda_{\delta}, \lambda\right)>\epsilon_{0}\right\} \leq \epsilon_{0} .
$$

Combining this with the second point, we deduce that for any $\epsilon_{1}>0$, there exists $\delta_{1}>0$ (locally uniform with respect to $\Omega$ ) such that for any $\delta \leq \delta_{1}$, we have

$$
\mathbb{P}\left\{\left|\frac{1}{\sqrt{\delta}} \frac{\mathbb{E}_{\Omega_{\delta} \backslash \lambda_{\delta}}^{\text {free }}\left[\sigma_{x_{\delta}} \sigma_{z_{\delta}}\right]}{\mathbb{E}_{\Omega_{\delta}}^{\left[r_{\delta}, \ell_{\delta}\right]_{+}}\left[\sigma_{x_{\delta}}\right]}-\frac{\left\langle\sigma_{x} \sigma_{z}\right\rangle_{\Omega \backslash \lambda}}{\left\langle\sigma_{x}\right\rangle_{\Omega}}\right|>\epsilon_{1}\right\} \leq \epsilon_{1} .
$$

Together with the third point, this allows to obtain that for any $\epsilon_{2}>0$ there exists $\delta_{2}>0$ (locally uniform with respect to $\Omega$ ) such that for any $\delta \leq \delta_{2}$, we have

$$
\left|\mathrm{E}_{\delta}^{\mathrm{A}}\left(\Omega_{\delta}, r_{\delta}, \ell_{\delta}, x_{\delta}, z\right)-\mathrm{E}^{\mathrm{A}}(\Omega, r, \ell, x, z)\right| \leq \epsilon_{2} .
$$

Indeed from the uniform bound 11.2 , we get that the contribution to the expectation of the event appearing in 11.3 can be made arbitrarily small.

\subsection{Proof of convergence of $\mathrm{E}_{\delta}^{\mathrm{B}}$.}

Proof of Proposition 14, part B. As in the proof of convergence of $\mathrm{E}_{\delta}^{\mathrm{A}}$, there are three ingredients (the types of convergence are as in Section 2.6). All statements are locally uniform with respect to $\Omega$.

- Convergence of the probability measure: from Theorem 32 , we have that the conditioned discrete FK interface $\tilde{\lambda}_{\delta}$ converges to the $\operatorname{SLE}(16 / 3 ;-8 / 3)$ trace $\tilde{\lambda}$.

- Convergence of the integrand: from Theorem 29, for any fixed curves $\tilde{\lambda}_{\delta}^{*}$ converging to $\tilde{\lambda}^{*}$ as $\delta \rightarrow 0$, we have

$$
\frac{1}{\sqrt{\delta}} \mathbb{E}_{\Omega_{\delta} \backslash \tilde{\lambda}_{\delta}^{*}}^{\left[r_{\delta}, x_{\delta}\right]_{+}}\left[\sigma_{z_{\delta}}\right] \rightarrow\left\langle\sigma_{z}\right\rangle_{\Omega \backslash \tilde{\lambda}^{*}}^{[r, \ell]_{+}},
$$

where the convergence is locally uniform with respect to $\tilde{\lambda}_{\delta}^{*}$.

- Uniform integrability of the integrand: for any $\epsilon>0$, let $\mathfrak{N}_{\delta}^{\epsilon}$ be the event that $\tilde{\lambda}_{\delta}$ hits the $\epsilon$-neighborhood $\mathbf{D}_{\Omega_{\delta}}\left(z_{\delta}, \epsilon\right)$ (as in defined in Lemma 24). Then, we get the uniform integrability from the following observations:

- On the complementary of $\mathfrak{N}_{\delta}^{\epsilon}$ (i.e. the event that $\tilde{\lambda}_{\delta}$ does not hit $\left.\mathbf{D}_{\Omega_{\delta}}\left(z_{\delta}, \epsilon\right)\right)$, the integrand $\frac{1}{\sqrt{\delta}} \mathbb{E}_{\Omega_{\delta} \backslash \tilde{\lambda}_{\delta}}^{\left[r_{\delta}, x_{\delta}\right]_{+}}\left[\sigma_{z_{\delta}}\right]$ is uniformly bounded with respect to $\delta>0$. This follows directly from Lemma 35 below.

- As $\epsilon \rightarrow 0, \frac{1}{\sqrt{\delta}} \mathbb{E}_{\Omega_{\delta} \backslash \tilde{\lambda}_{\delta}}^{\left[r_{\delta}, x_{+}\right]_{+}}\left[\sigma_{z_{\delta}} \mathbf{1}_{\mathfrak{N}_{\delta}^{\epsilon}}\right] \rightarrow 0$, uniformly with respect to $\delta$. We write $\mathfrak{N}_{\delta}^{\epsilon}=\mathfrak{A}_{\delta}^{\epsilon} \cup \mathfrak{A}_{\delta}^{\epsilon / 2} \cup \mathfrak{A}_{\delta}^{\epsilon / 4} \cup \ldots$, where for any $\eta>0$, we set $\mathfrak{A}_{\delta}^{\eta}:=$ $\mathfrak{N}_{\delta}^{\eta} \backslash \mathfrak{N}_{\delta}^{\eta / 2}$ 
- By Lemma 34, as $\eta \rightarrow 0$, the probability of $\mathfrak{A}_{\delta}^{\eta}$ behaves like $\mathcal{O}(\eta)$ uniformly with respect to $\delta$ by Lemma 35 , on $\mathfrak{A}_{\delta}^{\eta}$, the integrand is bounded by $\mathcal{O}\left(\frac{1}{\sqrt{\eta}}\right)$, uniformly with respect to $\delta$; hence $\frac{1}{\sqrt{\delta}} \mathbb{E}_{\Omega_{\delta} \backslash \tilde{\lambda}_{\delta}}^{\left[r_{\delta}, x_{j}\right]_{+}}\left[\sigma_{z_{\delta}} \mathbf{1}_{\mathfrak{N}_{\delta}^{\eta}}\right]=$ $\mathcal{O}(\sqrt{\eta})$. Summing this over the scales $\eta=\epsilon, \epsilon / 2, \epsilon / 4, \ldots, \delta$ (there are $\mathcal{O}\left(\log _{2}(\epsilon / \delta)\right)$ such scales), we get the result.

Using the exactly the same sequence of arguments as in the conclusion of the proof of part $\mathrm{A}$ of the proposition (in the previous subsection), we get the result.

Let us now give the two a priori estimates used in the proof above, which follow from results in [DHN11] (notice that we can directly use the results from this paper, as the boundary of $\Omega_{\delta}$ near $z_{\delta}$ is straight).

Lemma 34. The probability that $\tilde{\lambda}_{\delta}$ gets $\epsilon$-close to $z_{\delta}$ behaves like $\mathcal{O}(\epsilon)$, uniformly with respect to $\delta$, locally uniformly with respect to $\Omega$.

Proof. This follows readily from Proposition 12 in [DHN11]

Lemma 35. If $\Upsilon_{\delta} \subset \Omega_{\delta}$ is an $\epsilon$-neighborhood (in $\Omega_{\delta}$ ) of $z_{\delta}$, then

$$
\frac{1}{\sqrt{\delta}} \mathbb{E}_{\Upsilon_{\delta}}^{\left[r_{\delta}, x_{\delta}\right]_{+}}\left[\sigma_{z_{\delta}}\right]=\mathcal{O}\left(\frac{1}{\sqrt{\epsilon}}\right)
$$

uniformly with respect to $\delta$, locally uniformly with respect to $\Omega$.

Proof. This estimate follows directly from Proposition 18 in [DHN11].

\section{Computation of SLE expectations}

In this section, we compute the SLE expectations $\mathrm{E}^{\mathrm{A}}$ and $\mathrm{E}^{\mathrm{B}}$, defined as

$$
\begin{aligned}
& \mathrm{E}^{\mathrm{A}}(\Omega, r, \ell, x, z):=\mathbb{E}^{\mathrm{A}}\left[\frac{\left\langle\sigma_{x} \sigma_{z}\right\rangle_{\Omega \backslash \lambda}^{\text {free }}}{\left\langle\sigma_{x}\right\rangle_{\Omega}^{r,,]_{+}}}\right], \\
& \mathrm{E}^{\mathrm{B}}(\Omega, r, \ell, x, z):=\mathbb{E}^{\mathrm{B}}\left[\left\langle\sigma_{z}\right\rangle_{\Omega \backslash \tilde{\lambda}}^{[r, x]_{+}}\right],
\end{aligned}
$$

where $\lambda$ has the law of a chordal $\operatorname{SLE}(16 / 3)$ from $r$ to $s$ and $\tilde{\lambda}$ has the law of an $\operatorname{SLE}(16 / 3 ;-8 / 3)$ with starting point $r$, observation point $s$ and force point $x$, and where the integrands are as in Definition 13 . Let us now define two following Coulomb gas correlation functions which will be useful to compute $\mathrm{E}^{\mathrm{A}}$ and $\mathrm{E}^{\mathrm{B}}$.

Definition 36. For a simply connected domain $(\Omega, r, \ell, x, z)$ such that $z$ is on a smooth part of $\partial \Omega$ and given any conformal mapping $\eta_{\Omega}: \Omega \rightarrow \mathbb{H}$, we define $\mathfrak{m}^{\mathrm{A}}$ and $\mathfrak{m}^{\mathrm{B}}$ by:

$$
\begin{aligned}
\mathfrak{m}^{\mathrm{A}}(\Omega, r, \ell, x, z) & :=\left|\eta_{\Omega}^{\prime}(x)\right|^{\frac{1}{2}}\left|\eta_{\Omega}^{\prime}(z)\right|^{\frac{1}{2}} \mathfrak{m}^{\mathrm{A}}\left(\mathbb{H}, \eta_{\Omega}(r), \eta_{\Omega}(\ell), \eta_{\Omega}(x), \eta_{\Omega}(z)\right) \\
\mathfrak{m}^{\mathrm{B}}(\Omega, r, \ell, x, z) & :=\left|\eta_{\Omega}^{\prime}(z)\right|^{\frac{1}{2}} \mathfrak{m}^{\mathrm{B}}\left(\mathbb{H}, \eta_{\Omega}(r), \eta_{\Omega}(\ell), \eta_{\Omega}(x), \eta_{\Omega}(z)\right) \\
\mathfrak{m}^{\mathrm{A}}\left(\mathbb{H}, \eta_{\infty}, \eta_{0}, \eta_{1}, \eta_{2}\right) & :=C_{\mathrm{A}}^{\mathfrak{m}} \frac{\left(\eta_{0}-\eta_{\infty}\right)^{\frac{1}{2}}}{\left(\eta_{2}-\eta_{1}\right)^{\frac{1}{2}}\left(\eta_{2}-\eta_{\infty}\right)^{\frac{1}{2}}\left(\eta_{1}-\eta_{0}\right)^{\frac{1}{2}}} \int_{\chi}^{1} \frac{(1-\zeta)}{\zeta^{3 / 4}(\zeta-\chi)^{3 / 4}} \mathrm{~d} \zeta \\
\mathfrak{m}^{\mathrm{B}}\left(\mathbb{H}, \eta_{\infty}, \eta_{0}, \eta_{1}, \eta_{2}\right) & :=C_{\mathrm{B}}^{\mathfrak{m}} \frac{\left(\eta_{1}-\eta_{\infty}\right)^{1 / 2}}{\left(\eta_{2}-\eta_{\infty}\right)^{1 / 2}\left(\eta_{2}-\eta_{1}\right)^{1 / 2}} \cdot \frac{1}{\sqrt{\chi}} \int_{1-\chi}^{1} \frac{\zeta^{1 / 2}}{(\zeta-1+\chi)^{1 / 4}(1-\zeta)^{1 / 4}} \mathrm{~d} \zeta
\end{aligned}
$$


where $\chi$ is the cross-ratio defined by

$$
\chi:=\frac{\eta_{0}-\eta_{\infty}}{\eta_{2}-\eta_{0}} \frac{\eta_{2}-\eta_{1}}{\eta_{1}-\eta_{\infty}}
$$

and the constants $C_{\mathrm{A}}^{\mathfrak{m}}$ and $C_{\mathrm{B}}^{\mathfrak{m}}$ are defined by

$$
\begin{aligned}
C_{\mathrm{A}}^{\mathfrak{m}} & :=\frac{\sqrt{2}+1}{\pi^{3 / 2}} \frac{\Gamma\left(\frac{3}{4}\right)}{\Gamma\left(\frac{1}{4}\right)} \\
C_{\mathrm{B}}^{\mathfrak{m}} & :=\frac{2 \sqrt{1+\sqrt{2}}}{\pi^{3 / 2}} .
\end{aligned}
$$

Remark 37. These definitions are independent of the choice of $\eta_{\Omega}$.

With this notation, Proposition 16 simply becomes:

Proposition (Proposition 16). We have

$$
\begin{aligned}
\mathrm{E}^{\mathrm{A}}(\Omega, r, \ell, x, z) & =\frac{\mathfrak{m}^{\mathrm{A}}(\Omega, r, \ell, x, z)}{\left\langle\sigma_{x}\right\rangle_{\Omega}^{[r, \ell]_{+}}} \\
\mathrm{E}^{\mathrm{B}}(\Omega, r, \ell, x, z) & =\mathfrak{m}^{\mathrm{B}}(\Omega, r, \ell, x, z) \\
\Phi(\Omega, r, \ell, x, z) & =\mathrm{E}^{\mathrm{A}}(\Omega, r, \ell, x, z)+\mathrm{E}^{\mathrm{B}}(\Omega, r, \ell, x, z) .
\end{aligned}
$$

Notice that the right-hand side of 12.1 is well-defined also when $x$ is on a rough boundary: the derivative terms in the definition of $\mathfrak{m}^{\mathrm{A}}$ and $\left\langle\sigma_{x}\right\rangle$ cancel each other. On the other hand, it is required that $z$ is on a smooth part of $\partial \Omega$.

Proof. To compute $\mathrm{E}^{\mathrm{A}}$ and $\mathrm{E}^{\mathrm{B}}$, notice that by conformal invariance of SLE and by conformal covariance of the correlation functions in the integrand, it is sufficient to compute $\mathrm{E}^{\mathrm{A}}$ and $\mathrm{E}^{\mathrm{B}}$ on the upper half-plane, and that we can choose the locations of three of the four boundary marked points. Hence the result follows from the computation for $\mathrm{E}^{\mathrm{A}}(\mathbb{H},-1,0,1, y)$, for any $y \in(-\infty,-1)$ performed in Section 12.1 , and the one of $\mathrm{E}^{\mathrm{B}}(\mathbb{H}, \infty, 0,1, w)$ for any $w \in(1, \infty)$, performed in Section 12.2 .

To obtain the formula for $\Phi$, we use the following hypergeometric representations of $\mathfrak{m}^{\mathrm{A}}$ and $\mathfrak{m}^{\mathrm{B}}$ :

$$
\begin{aligned}
\mathfrak{m}^{\mathrm{A}}\left(\mathbb{H}, \eta_{\infty}, \eta_{0}, \eta_{1}, \eta_{2}\right) & =\frac{16 C_{\mathrm{A}}^{\mathfrak{m}}}{5} \frac{\left(\eta_{0}-\eta_{\infty}\right)^{\frac{1}{2}}\left(\eta_{2}-\eta_{\infty}\right)^{\frac{3}{4}}\left(\eta_{1}-\eta_{0}\right)^{\frac{3}{4}}}{\left(\eta_{1}-\eta_{\infty}\right)^{\frac{5}{4}}\left(\eta_{2}-\eta_{0}\right)^{\frac{5}{4}}\left(\eta_{2}-\eta_{1}\right)^{\frac{1}{2}}} F_{1}\left(\frac{3}{4}, 2 ; \frac{9}{4} ; 1-\chi\right) \\
\mathfrak{m}^{\mathrm{B}}\left(\mathbb{H}, \eta_{\infty}, \eta_{0}, \eta_{1}, \eta_{2}\right) & =\frac{2 \Gamma\left(\frac{3}{4}\right)^{2} C_{\mathrm{B}}^{\mathfrak{m}}}{\sqrt{\pi}} \frac{\left(\eta_{1}-\eta_{\infty}\right)^{\frac{1}{2}}}{\left(\eta_{2}-\eta_{\infty}\right)^{\frac{1}{2}}\left(\eta_{2}-\eta_{1}\right)^{\frac{1}{2}}}{ }_{2} F_{1}\left(-\frac{1}{2}, \frac{3}{4} ; \frac{3}{2} ; \chi\right),
\end{aligned}
$$

where the branch of the hypergeometric function ${ }_{2} F_{1}$ on $\mathbb{C} \backslash[1, \infty]$ is the usual one. Then, from the formula in [AbSt64, Eq. 15.3.6], we get

$$
\begin{aligned}
\mathfrak{m}^{\mathrm{B}}\left(\mathbb{H}, \eta_{\infty}, \eta_{0}, \eta_{1}, \eta_{2}\right)= & \frac{\Gamma\left(\frac{1}{4}\right) \Gamma\left(\frac{3}{4}\right)}{4} C_{\mathrm{B}}^{\mathfrak{m}} \frac{\left(\eta_{1}-\eta_{\infty}\right)^{\frac{1}{2}}}{\left(\eta_{2}-\eta_{\infty}\right)^{\frac{1}{2}}\left(\eta_{2}-\eta_{1}\right)^{\frac{1}{2}}}{ }_{2} F_{1}\left(-\frac{1}{2}, \frac{3}{4} ;-\frac{1}{4} ; 1-\chi\right) \\
& -\frac{8 \Gamma\left(\frac{3}{4}\right)^{2}}{5 \sqrt{\pi}} C_{\mathrm{B}}^{\mathfrak{m}} \frac{(1-\chi)^{\frac{5}{4}}\left(\eta_{1}-\eta_{\infty}\right)^{\frac{1}{2}}}{\left(\eta_{2}-\eta_{\infty}\right)^{\frac{1}{2}}\left(\eta_{2}-\eta_{1}\right)^{\frac{1}{2}}} F_{1}\left(\frac{3}{4}, 2 ; \frac{9}{4} ; 1-\chi\right) .
\end{aligned}
$$




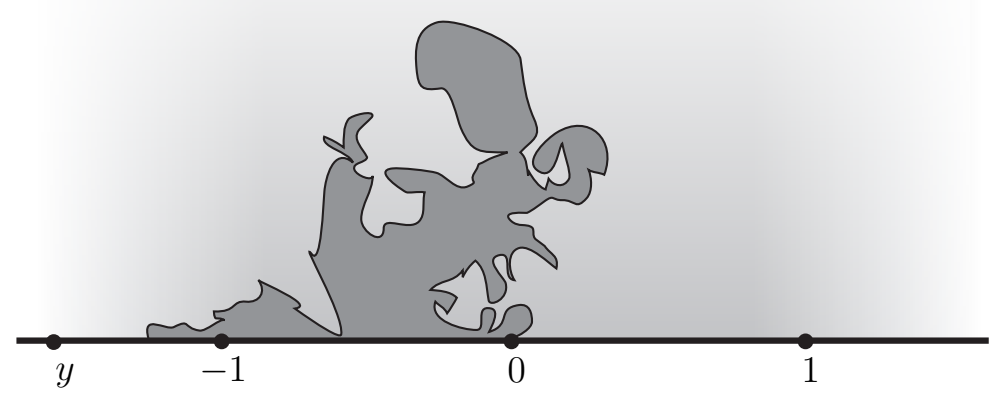

FigurE 12.1. Setup for $\mathrm{E}^{\mathrm{A}}(\mathbb{H},-1,0,1, y)$

Recalling that

$$
\left\langle\sigma_{\eta_{1}}\right\rangle_{\mathbb{H}}^{\left[\eta_{\infty}, \eta_{0}\right]_{+}}=\sqrt{\frac{\sqrt{2}+1}{2 \pi}} \frac{\left(\eta_{0}-\eta_{\infty}\right)^{\frac{1}{2}}}{\left(\eta_{1}-\eta_{0}\right)^{\frac{1}{2}}\left(\eta_{1}-\eta_{\infty}\right)^{\frac{1}{2}}}
$$

we notice a cancellation in the sum

$$
\frac{\mathfrak{m}^{\mathrm{A}}\left(\mathbb{H}, \eta_{\infty}, \eta_{0}, \eta_{1}, \eta_{2}\right)}{\left\langle\sigma_{\eta_{1}}\right\rangle_{\mathbb{H}}^{\left[\eta_{\infty}, \eta_{0}\right]_{+}}}+\mathfrak{m}^{\mathrm{B}}\left(\mathbb{H}, \eta_{\infty}, \eta_{0}, \eta_{1}, \eta_{2}\right)
$$

and then write the result of the sum, which simplifies to

$$
\sqrt{\frac{\sqrt{2}+1}{2 \pi}} \frac{\left(\eta_{1}-\eta_{\infty}\right)^{\frac{1}{2}}}{\left(\eta_{2}-\eta_{\infty}\right)^{\frac{1}{2}}\left(\eta_{2}-\eta_{1}\right)^{\frac{1}{2}}}{ }_{2} F_{1}\left(-\frac{1}{2}, \frac{3}{4} ;-\frac{1}{4} ; 1-\chi\right) .
$$

Finally, using that

$$
{ }_{2} F_{1}\left(-\frac{1}{2}, \frac{3}{4} ;-\frac{1}{4} ; z\right)=\frac{1+z}{\sqrt{1-z}}
$$

and mapping conformally to the strip $\mathbb{S}$, we obtain $\mathrm{E}^{\mathrm{A}}+\mathrm{E}^{\mathrm{B}}=\Phi$.

\subsection{Computation of $\mathrm{E}^{\mathrm{A}}$.}

Proposition 38. For any $y \in(-\infty,-1)$, we have

$$
\mathrm{E}^{\mathrm{A}}(\mathbb{H},-1,0,1, y)=\frac{\mathfrak{m}^{\mathrm{A}}(\mathbb{H},-1,0,1, y)}{\left\langle\sigma_{1}\right\rangle_{\mathbb{H}}^{[-1,0]_{+}}}
$$

Proof. By SLE coordinate change (Section 10.1), we can take $\mathrm{E}^{\mathrm{A}}$ to be the expectation of

$$
\mathbb{E}\left[\frac{\left\langle\sigma_{1} \sigma_{y}\right\rangle_{\mathbb{H} \backslash \lambda}^{\text {free }}}{\left\langle\sigma_{1}\right\rangle_{\mathbb{H}}^{[-1,0]_{+}}}\right]
$$

where $\lambda$ has the law of an $\operatorname{SLE}(\kappa ; \kappa-6)$ (with $\kappa=16 / 3$ ) in $\mathbb{H}$ with source 0 , observation point $\infty$ and force point -1 ; again the integrand is set to 0 if $\lambda$ disconnects $y$ 
from 1 before disconnecting -1 from $\infty$. Parametrize $\lambda$ by the standard half-plane capacity viewed from $\infty$. For each $t \geq 0$, let $H_{t}$ be the unbounded connected component of $\mathbb{H} \backslash \lambda[0, t]$. Let $\tau^{\mathrm{A}}$ be the (almost surely finite) first time when 1 and -1 are disconnected by $\lambda$. Define $\left(\mathbf{M}_{t}^{\mathrm{A}}\right)_{t \geq 0}$ by

$$
\mathbf{M}_{t}^{\mathrm{A}}:=\mathfrak{m}^{\mathrm{A}}\left(H_{t \wedge \tau^{\mathrm{A}}},-1, \lambda\left(t \wedge \tau^{\mathrm{A}}\right), 1, y\right) .
$$

By conformal covariance, if we denote by $g_{t}: H_{t} \rightarrow \mathbb{H}$ the conformal mapping with normalization $\lim _{z \rightarrow \infty} g_{t}(z)-z=0$, we have, for $t<\tau^{\mathrm{A}}$,

$$
\mathbf{M}_{t}^{\mathrm{A}}=\sqrt{g_{t}^{\prime}(y)} \sqrt{g_{t}^{\prime}(1)} \mathfrak{m}^{\mathrm{A}}\left(\mathbb{H}, g_{t}(-1), U_{t}, g_{t}(1), g_{t}(y)\right),
$$

where $U_{t}=g_{t}(\lambda(t))$ is the driving force of the Loewner chain. The process $\mathbf{M}_{t}^{\mathrm{A}}$ has the following properties, shown further in this subsection:

- $\mathbf{M}_{t}^{\mathrm{A}}$ is a local martingale (Lemma 39);

- $\mathbf{M}_{t}^{\mathrm{A}}$ is bounded (Lemma 40);

- $\mathbf{M}_{t}^{\mathrm{A}}$ has the correct endvalue (Lemma 41):

$$
\mathbf{M}_{\tau^{\mathrm{A}}}^{\mathrm{A}}=\left\langle\sigma_{1} \sigma_{y}\right\rangle_{\mathbb{H} \backslash \lambda\left[0, \tau^{\mathrm{A}}\right]}^{\text {free }},
$$

where the right-hand side is zero if $y$ and 1 are disconnected by $\lambda\left[0, \tau^{\mathrm{A}}\right]$.

From these three lemmas, we deduce that $\mathbf{M}_{t}^{\mathrm{A}}$ is a uniformly integrable martingale and by the optional stopping theorem, we hence obtain

$$
\mathrm{E}^{\mathrm{A}}(\mathbb{H},-1,0,1, y)=\mathbb{E}\left[\frac{\mathbf{M}_{\tau^{\mathrm{A}}}^{\mathrm{A}}}{\left\langle\sigma_{1}\right\rangle_{\mathbb{H}}}\right]=\frac{\mathbf{M}_{0}^{\mathrm{A}}}{\left\langle\sigma_{1}\right\rangle_{\mathbb{H}}}=\frac{\mathfrak{m}^{\mathrm{A}}(\mathbb{H},-1,1, y)}{\left\langle\sigma_{1}\right\rangle_{\mathbb{H}}},
$$

which concludes the proof of the proposition.

Let us show the three lemmas used in the proof of Proposition 38.

Lemma 39. $\mathbf{M}_{t}^{\mathrm{A}}$ is a local martingale.

Proof. With the same notation as in the proof of Proposition 38, for $t<\tau_{\mathrm{A}}$ we have

$$
\mathbf{M}_{t}^{\mathrm{A}}=\sqrt{g_{t}^{\prime}(y)} \sqrt{g_{t}^{\prime}(1)} \mathfrak{m}^{\mathrm{A}}\left(\mathbb{H}, g_{t}(-1), U_{t}, g_{t}(1), g_{t}(y)\right),
$$

where

$$
\begin{aligned}
\mathrm{d} g_{t}(z) & =\frac{2 \mathrm{~d} t}{g_{t}(z)-U_{t}}, \\
\mathrm{~d} U_{t} & =\sqrt{\kappa} \mathrm{d} B_{t}+\frac{\rho \mathrm{d} t}{U_{t}-g_{t}(-1)}
\end{aligned}
$$

where $\kappa=16 / 3$ and $\rho=-2 / 3$. By Itô's calculus, we get that the drift of $\mathbf{M}_{t}^{\mathrm{A}}$ is proportional to

$$
\begin{aligned}
& \left(-\frac{1}{\left(\eta_{1}-\eta_{0}\right)^{2}}-\frac{1}{\left(\eta_{2}-\eta_{0}\right)^{2}}+\frac{2}{\eta_{\infty}-\eta_{0}} \frac{\partial}{\partial \eta_{\infty}}+\frac{2}{\eta_{1}-\eta_{0}} \frac{\partial}{\partial \eta_{1}}+\right. \\
& \left.\frac{2}{\eta_{2}-\eta_{0}} \frac{\partial}{\partial \eta_{2}}-\frac{2}{3} \frac{1}{\eta_{0}-\eta_{\infty}} \frac{\partial}{\partial \eta_{0}}+\frac{8}{3} \frac{\partial^{2}}{\partial \eta_{0}^{2}}\right) \mathfrak{m}^{\mathrm{A}}\left(\mathbb{H}, \eta_{\infty}, \eta_{0}, \eta_{1}, \eta_{2}\right),
\end{aligned}
$$

evaluated at $\eta_{\infty}=g_{t}(-1), \eta_{0}=U_{t}, g_{t}(y), g_{t}(1)$. From the explicit form of $\mathfrak{m}^{\mathrm{A}}$, it is easy to check that this expression vanishes.

Lemma 40. $\mathbf{M}_{t}^{\mathrm{A}}$ is bounded. 
Proof. By Remark 26, we have the following monotonicity property for the integrand

$$
\left\langle\sigma_{1} \sigma_{y}\right\rangle_{\mathbb{H} \backslash \lambda[0, t]}^{\text {free }} \leq\left\langle\sigma_{1} \sigma_{y}\right\rangle_{\mathbb{H}}^{\text {free }}
$$

for any $\lambda$. Hence, we obtain that $\mathbf{M}_{t}^{\mathrm{A}}$ is bounded by the right-hand side.

Lemma 41. On stopping time $\tau^{\mathrm{A}}, \mathbf{M}_{t}^{\mathrm{A}}$ has the endvalue

$$
\mathbf{M}_{\tau^{\mathrm{A}}}^{\mathrm{A}}=\left\langle\sigma_{1} \sigma_{y}\right\rangle_{\mathbb{H} \backslash \lambda\left[0, \tau^{\mathrm{A}}\right]}^{\text {free }} .
$$

Proof. Recall that

$$
\mathbf{M}_{t}^{\mathrm{A}}=\sqrt{g_{t}^{\prime}(1)} \sqrt{g_{t}^{\prime}(y)} \mathfrak{m}^{\mathrm{A}}\left(\mathbb{H} ; g_{t}(-1), U_{t}, g_{t}(1), g_{t}(y)\right) .
$$

We should show that

$$
\lim _{t \rightarrow \tau^{\mathrm{A}}} \mathbf{M}_{t}^{\mathrm{A}}=\left\langle\sigma_{1} \sigma_{y}\right\rangle_{\mathbb{H} \backslash \lambda\left[0, \tau^{\mathrm{A}}\right]}^{\text {free }}
$$

Denote by $\chi_{t}$ the cross-ratio defined by

$$
\chi_{t}:=\frac{U_{t}-g_{t}(-1)}{U_{t}-g_{t}(y)} \frac{g_{t}(1)-g_{t}(y)}{g_{t}(1)-g_{t}(-1)} .
$$

As $t \rightarrow \tau^{\mathrm{A}}$, there are three possibilities:

- $\lambda$ reaches the interval $(y,-1]:$ in this case, $U_{t}-g_{t}(-1) \rightarrow 0$ while other distances remain positive, so in particular $\chi_{t} \rightarrow 0$. Using the same hypergeometric representation as in the proof of Proposition 16 and the identity (see [AbSt64, Eq 15.3.6])

$$
\begin{aligned}
{ }_{2} F_{1}\left(\frac{3}{4}, 2 ; \frac{9}{4} ; 1-\chi\right)= & -\frac{5}{4}{ }_{2} F_{1}\left(\frac{3}{4}, 2 ; \frac{3}{2} ; \chi\right) \\
& +\frac{5 \sqrt{\pi}}{16} \frac{\Gamma\left(\frac{1}{4}\right)}{\Gamma\left(\frac{3}{4}\right)} \chi^{-\frac{1}{2}}{ }_{2} F_{1}\left(\frac{3}{2}, \frac{1}{4} ; \frac{1}{2} ; \chi\right),
\end{aligned}
$$

we see that in this case, $M_{t}^{\mathrm{A}}$ tends to

$$
C_{\mathrm{A}}^{\mathfrak{m}} \frac{\sqrt{\pi} \Gamma\left(\frac{1}{4}\right)}{\Gamma\left(\frac{3}{4}\right)} \frac{\sqrt{g_{\tau^{\mathrm{A}}}^{\prime}(1)} \sqrt{g_{\tau^{\mathrm{A}}}^{\prime}(y)}}{g_{\tau^{\mathrm{A}}}(1)-g_{\tau^{\mathrm{A}}}(y)},
$$

which is equal to $\left\langle\sigma_{1} \sigma_{y}\right\rangle_{\mathbb{H} \backslash \lambda\left[0, \tau^{\mathrm{A}}\right]}^{\text {free }}$.

- $\lambda$ reaches the interval $[1, \infty):$ in this case, $g_{t}(1)-U_{t} \rightarrow 0$ while other distances remain positive, so $\chi_{t} \rightarrow 1$. Observing that $\left|g_{t}^{\prime}(1)\right| \leq 1$ and $\left|g_{t}^{\prime}(y)\right| \leq 1$ and using the explicit expression for $\mathfrak{m}^{\mathrm{A}}$, we see that in this case $\mathbf{M}_{t}^{\mathrm{A}} \rightarrow 0$. We have that 1 and $y$ get disconnected from each other by $\lambda\left[0, \tau^{\mathrm{A}}\right]$, so

$$
\lim _{t \rightarrow \tau^{\mathrm{A}}} \mathbf{M}_{t}^{\mathrm{A}}=0=\left\langle\sigma_{1} \sigma_{y}\right\rangle_{\mathbb{H} \backslash \lambda\left[0, \tau^{\mathrm{A}}\right]}^{\text {free }} .
$$

- $\lambda$ reaches the interval $(-\infty, y]$ : in this case, both $U_{t}-g_{t}(y)$ and $U_{t}-g_{t}(-1)$ tend to zero and considerations of harmonic measure show that

$$
\frac{U_{t}-g_{t}(-1)}{U_{t}-g_{t}(y)} \rightarrow 1
$$

so $\chi_{t} \rightarrow 1$. As in the previous case, one concludes that both the limit of $\mathbf{M}_{t}^{\mathrm{A}}$ and the spin correlation $\left\langle\sigma_{1} \sigma_{y}\right\rangle_{\mathbb{H} \backslash \lambda\left[0, \tau^{\mathrm{A}}\right]}^{\text {free }}$ are zero. 


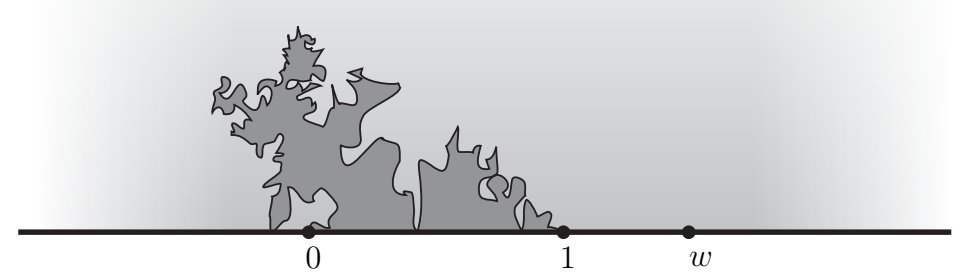

Figure 12.2. Setup for $\mathrm{E}^{\mathrm{B}}(\mathbb{H}, \infty, 0,1, w)$

\subsection{Computation of $\mathrm{E}^{\mathrm{B}}$.}

Proposition 42. For any $w>1$, we have

$$
\mathrm{E}^{\mathrm{B}}(\mathbb{H}, \infty, 0,1, w)=\mathfrak{m}^{\mathrm{B}}(\mathbb{H}, \infty, 0,1, w) .
$$

Proof. The proof is similar to the one of Proposition 38 . Let $\tilde{\lambda}$ have the law of an SLE $(16 / 3,-8 / 3)$ trace with source point 0 , observation point $\infty$ and force point 1. Consider the standard half-plane capacity parametrization of the curve and for each $t \geq 0$, let $H_{t}$ be the unbounded connected component of $\mathbb{H} \backslash \lambda[0, t]$. Let $\tau^{\mathrm{B}}$ be the (almost surely finite) hitting time of 1 . Let $\mathbf{M}_{t}^{\mathrm{B}}$ be defined by

$$
\mathbf{M}_{t}^{\mathrm{B}}:=\mathfrak{m}^{\mathrm{B}}\left(H_{t \wedge \tau^{\mathrm{B}}}, \infty, \tilde{\lambda}\left(t \wedge \tau^{\mathrm{B}}\right), 1, w\right) .
$$

By conformal covariance, if we denote by $g_{t}: H_{t} \rightarrow \mathbb{H}$ the conformal mapping with normalization $\lim _{z \rightarrow \infty} g_{t}(z)-z=0$, we have, for $t<\tau_{\mathrm{B}}$

$$
\mathbf{M}_{t}^{\mathrm{B}}=\sqrt{g_{t}^{\prime}(w)} \mathfrak{m}^{\mathrm{B}}\left(\mathbb{H}, \infty, U_{t}, g_{t}(1), g_{t}(w)\right),
$$

where $U_{t}=g_{t}(\lambda(t))$ is the driving process of the Loewner chain. We then have the following properties, shown later in this subsection

- $\mathbf{M}_{t}^{\mathrm{B}}$ is a local martingale (Lemma 43 );

- $\mathbf{M}_{t}^{\mathrm{B}}$ is uniformly integrable (Lemma 44);

- $\mathbf{M}_{t}^{\mathrm{B}}$ has the correct endvalue (Lemma 45):

$$
\mathbf{M}_{\tau^{\mathrm{B}}}^{\mathrm{B}}=\left\langle\sigma_{w}\right\rangle_{\mathbb{H} \backslash \tilde{\lambda}\left[0, \tau^{\mathrm{B}}\right]}^{[-\infty, 1]_{+}} .
$$

From these properties, we deduce, by the optional stopping theorem:

$$
\mathrm{E}^{\mathrm{B}}(\mathbb{H}, \infty, 0,1, w)=\mathbb{E}\left[\mathbf{M}_{\tau^{\mathrm{B}}}^{\mathrm{B}}\right]=\mathbf{M}_{0}^{\mathrm{B}}=\mathfrak{m}^{\mathrm{B}}(\mathbb{H}, \infty, 0,1, w) .
$$

Lemma 43. $\mathbf{M}_{t}^{\mathrm{B}}$ is a local martingale.

Proof. Writing, as in the proof of Proposition 42, for $t<\tau^{\mathrm{B}}$,

$$
\mathbf{M}_{t}^{\mathrm{B}}=\sqrt{g_{t}^{\prime}(w)} \mathfrak{m}_{t}^{\mathrm{B}}\left(\mathbb{H}, \infty, U_{t}, g_{t}(1), g_{t}(w)\right),
$$


and using that

$$
\begin{aligned}
\mathrm{d} g_{t}(z) & =\frac{2 \mathrm{~d} t}{g_{t}(z)-U_{t}} \\
\mathrm{~d} U_{t} & =\sqrt{\kappa} \mathrm{d} B_{t}+\frac{\rho \mathrm{d} t}{U_{t}-g_{t}(1)}
\end{aligned}
$$

where $\kappa=16 / 3$ and $\rho=-8 / 3$. Itô's calculus gives that the drift of $\mathbf{M}_{t}^{\mathrm{B}}$ is proportional to

$$
\begin{array}{r}
\left(-\frac{1}{\left(\eta_{2}-\eta_{0}\right)^{2}}+\frac{2}{\eta_{1}-\eta_{0}} \frac{\partial}{\partial \eta_{1}}+\frac{2}{\eta_{2}-\eta_{0}} \frac{\partial}{\partial \eta_{2}}-\frac{8}{3} \frac{1}{\eta_{0}-\eta_{1}} \frac{\partial}{\partial \eta_{0}}+\frac{8}{3} \frac{\partial^{2}}{\partial \eta_{0}^{2}}\right) \\
\mathfrak{m}^{\mathrm{B}}\left(\mathbb{H}, \infty, \eta_{0}, \eta_{1}, \eta_{2}\right),
\end{array}
$$

evaluated at $\eta_{0}=U_{t}, \eta_{1}=g_{t}(1), \eta_{2}=g_{t}(w)$. From the explicit formula for $\mathfrak{m}^{\mathrm{B}}$, we get that this expression is zero.

Lemma 44. $\mathbf{M}_{t}^{\mathrm{B}}$ is uniformly integrable

Proof. The proof of this is completely analogous to the one of the convergence of $\mathrm{E}_{\delta}^{\mathrm{B}}$ in Section 11.2. It indeed follows from Lemmas 34 and 35 , passed to the $\delta \rightarrow 0$ limit, using Theorems 25 and 27. the probability that the curve $\tilde{\lambda}$ gets $\epsilon$-close to $w$ decays like $\mathcal{O}(\epsilon)$, while the blow-up of the integrand as the curve gets $\epsilon$-close to $w$ is only $\mathcal{O}\left(\frac{1}{\sqrt{\epsilon}}\right)$.

Lemma 45. $\mathbf{M}_{t}^{\mathrm{B}}$ has the endvalue:

$$
\mathbf{M}_{\tau^{\mathrm{B}}}^{\mathrm{B}}=\left\langle\sigma_{w}\right\rangle_{\mathbb{H} \backslash \tilde{\lambda}\left[0, \tau^{\mathrm{B}}\right]}^{[-\infty, 1]_{+}} .
$$

Proof. By continuity, we should show that

$$
\mathbf{M}_{t}^{\mathrm{B}} \underset{t \rightarrow \tau^{\mathrm{B}}}{\longrightarrow}\left\langle\sigma_{w}\right\rangle_{\mathbb{H} \backslash \tilde{\lambda}\left[0, \tau^{\mathrm{B}}\right]}^{[-\infty, 1]_{+}}
$$

By conformal covariance, we have that

$$
\mathbf{M}_{t}^{\mathrm{B}}=\sqrt{g_{t}^{\prime}(w)} \mathfrak{m}^{\mathrm{B}}\left(\mathbb{H}, \infty, U_{t}, g_{t}(1), g_{t}(w)\right)
$$

and since as $t \rightarrow \tau^{\mathrm{B}}$, we have $g_{t}(1)-U_{t} \rightarrow 0$ (as the tip of the curve $\tilde{\lambda}(t)$ tends to 1) and $g_{t}(w)-U_{t}$ remains bounded away from 0 , it is enough to show (again by conformal covariance) that for any $w>1$,

$$
\lim _{z \rightarrow 0_{+}} \mathfrak{m}^{\mathrm{B}}(\mathbb{H}, \infty, 0, z, w)=\left\langle\sigma_{w}\right\rangle_{\mathbb{H}}^{[-\infty, 0]_{+}}
$$

To obtain these asymptotics, we use the same hypergeometric representation of $\mathfrak{m}^{\mathrm{B}}$ and then the same decomposition formula for the hypergeometric function ${ }_{2} F_{1}$ as in 
the Proof of Proposition 16 :

$$
\begin{aligned}
\mathfrak{m}^{\mathrm{B}}(\mathbb{H}, \infty, 0, z, w) & =\frac{2 \Gamma\left(\frac{3}{4}\right)^{2} C_{\mathrm{B}}^{\mathfrak{m}}}{\sqrt{\pi}} \frac{1}{(w-z)^{\frac{1}{2}}}{ }_{2} F_{1}\left(-\frac{1}{2}, \frac{3}{4} ; \frac{3}{2} ; 1-\frac{z}{w}\right) \\
& =\frac{\Gamma\left(\frac{1}{4}\right) \Gamma\left(\frac{3}{4}\right) C_{\mathrm{B}}^{\mathfrak{m}}}{4} \frac{1}{(w-z)^{\frac{1}{2}}}{ }_{2} F_{1}\left(-\frac{1}{2}, \frac{3}{4} ;-\frac{1}{4} ; \frac{z}{w}\right) \\
& -\frac{8 \Gamma\left(\frac{3}{4}\right)^{2} C_{\mathrm{B}}^{\mathfrak{m}}}{5 \sqrt{\pi}} \frac{1}{(w-z)^{\frac{1}{2}}}\left(\frac{z}{w}\right)^{\frac{5}{4}}{ }_{2} F_{1}\left(\frac{3}{4}, 2 ; \frac{9}{4} ; \frac{z}{w}\right) \\
& \longrightarrow \frac{\Gamma\left(\frac{1}{4}\right) \Gamma\left(\frac{3}{4}\right) C_{\mathrm{B}}^{\mathfrak{m}}}{4} \frac{1}{\sqrt{w}} \\
& =\sqrt{\frac{\sqrt{2}+1}{2 \pi}} \cdot \frac{1}{\sqrt{w}} \\
& =\left\langle\sigma_{w}\right\rangle_{\mathbb{H}}^{[-\infty, 0]_{+}} .
\end{aligned}
$$

\section{Discrete Complex Analysis}

In this section, we show the convergence of the ratios of elementary Ising correlation functions to CFT correlation functions stated in Section 9 (Theorem 29).

Theorem (Theorem 29). Let $(\Theta, y, t, x),(\tilde{\Theta}, \tilde{y}, \tilde{t}, x)$ and $(\Xi, x, s)$ be domains which coincide in a neighborhood of the boundary point $x$. Suppose that $s$ lies on a vertical part $\mathfrak{v}$ of $\partial \Xi$. Let $\left(\Theta_{\delta}, y_{\delta}, t_{\delta}, x_{\delta}\right),\left(\tilde{\Theta}_{\delta}, \tilde{y}_{\delta}, \tilde{t}_{\delta}, x_{\delta}\right)$ and $\left(\Xi_{\delta}, x_{\delta}, s_{\delta}\right)$ be discretizations of these domains coinciding in a neighborhood of $x_{\delta}$, converging to their continuous counterparts in the sense of the metric of Section 2.6. Suppose that for each $\delta>0$, $\partial \Xi_{\delta}$ contains a vertical part $\mathfrak{v}_{\delta}$ around $s_{\delta}$ and that as $\delta \rightarrow 0, \mathfrak{v}_{\delta}$ converges to $\mathfrak{v}$. Then we have

$$
\begin{aligned}
\frac{1}{\sqrt{\delta}} \frac{\mathbb{E}_{\Xi_{\delta}}^{\text {free }}\left[\sigma_{x_{\delta}} \sigma_{s_{\delta}}\right]}{\mathbb{E}_{\Theta_{\delta}}^{\left[y_{\delta}, t_{\delta}\right]_{+}}\left[\sigma_{x_{\delta}}\right]} \underset{\delta \rightarrow 0}{\longrightarrow} \frac{\left\langle\sigma_{x} \sigma_{s}\right\rangle_{\Xi}^{\text {free }}}{\left\langle\sigma_{x}\right\rangle_{\Theta}^{[y, t]_{+}}} \\
\frac{\mathbb{E}_{\tilde{\Theta}_{\delta}}^{\left[\tilde{y}_{\delta}, \tilde{t}_{\delta}\right]_{+}}\left[\sigma_{x_{\delta}}\right]}{\mathbb{E}_{\Theta_{\delta}}^{\left[y_{\delta}, t_{\delta}\right]_{+}}\left[\sigma_{x_{\delta}}\right]} \underset{\delta \rightarrow 0}{\longrightarrow} \frac{\left\langle\sigma_{x}\right\rangle_{\tilde{\Theta}}^{\left[\tilde{y}_{\delta}, \tilde{t}_{\delta}\right]_{+}}}{\left\langle\sigma_{x}\right\rangle_{\Theta}^{\left[y_{\delta}, t_{\delta}\right]_{+}}}
\end{aligned}
$$

The convergence is locally uniform with respect to the domains.

The proof of Theorem 29 is given in Section 13.9. The key tool, introduced in [Smi10a] and further developed in [ChSm11, ChSm09, HoSm10b] is (a type of) discrete complex analysis. More precisely, the structure of this section is as follows:

- In Section 13.1, we precisely define the graphs and notations that are suited for the discrete complex analysis tools that we use.

- In Section 13.2, we define and give basic properties of the discrete holomorphic observables that are instrumental to compute the discrete correlation functions of Theorem 29. 
- In Section 13.3, we obtain the discrete correlation functions as the boundary values of the discrete holomorphic observables.

- In Section 13.4, we formulate a discrete Riemann boundary value problem which provides a convenient local representation of the discrete holomorphic observables in terms of a convolution kernel.

- In Section 13.5, we introduce the continuous counterpart of the discrete holomorphic observables.

- In Section 13.6, we obtain the CFT correlation functions appearing in Theorem 29 as boundary values of the continuous holomorphic observables.

- In Section 13.7, we formulate the continuous Riemann boundary value problem which gives a local representation of the continuous observables.

- In Section 13.8, we derive the convergence (in the bulk and on straight parts of the boundary) of the discrete holomorphic observables to the continuous ones.

- In Section 13.9, we show Theorem 29. To do this, we extend to the boundary the convergence results of Section 13.8 for appropriate ratios.

- In Section 13.10, we show the a priori estimates used in Section 13.9 to prove Theorem 29.

13.1. Graphs, notation and definitions. Let $\Omega_{\delta}$ be a discrete vertex domain: a connected subgraph of the square grid $\mathbb{C}_{\delta}:=\delta \mathbb{Z}^{2}$.

- We denote by $\mathcal{V}_{\Omega_{\delta}}$ the set of vertices and $\mathcal{E}_{\Omega_{\delta}}$ the set of edges of $\Omega_{\delta}$.

- We denote by $\operatorname{Int}\left(\Omega_{\delta}\right)$ the complex domain bounded by the dual circuit made of edges of $\mathcal{E}_{\mathbb{C}_{\delta}^{*} \backslash \Omega_{\delta}^{*}}\left(\right.$ see Figure 13.1), by $\partial \hat{\Omega}_{\delta}$ the set of its prime ends and by $\hat{\Omega}_{\delta}$ its Carathédory compactification (for a definition of these notions, see [Pom92, Chapters 1,2], for instance).

- We denote by $\partial \mathcal{E}_{\Omega_{\delta}}$ the set of edges of $\mathcal{E}_{\mathbb{C}_{\delta}} \backslash \mathcal{E}_{\Omega_{\delta}}$ that are incident to a vertex of $\mathcal{V}_{\Omega_{\delta}}$, counted with multiplicity: if an edge $e \in \mathcal{E}_{\mathbb{C}_{\delta}} \backslash \mathcal{E}_{\Omega_{\delta}}$ is incident to two vertices of $\mathcal{V}_{\Omega_{\delta}}$, it appears as two distinct elements of $\partial \mathcal{E}_{\Omega_{\delta}}$.

- We denote by $\partial \mathcal{V}_{\Omega_{\delta}}$ the set of vertices of $\mathcal{V}_{\mathbb{C}_{\delta}} \backslash \mathcal{V}_{\Omega_{\delta}}$ incident to $\partial \mathcal{E}_{\Omega_{\delta}}$, counted with multiplicity: if two edges of $\partial \mathcal{E}_{\delta}$ are incident to a vertex $v \in \mathcal{V}_{\mathbb{C}_{\delta}} \backslash \mathcal{V}_{\Omega_{\delta}}$, that vertex counts as two elements of $\partial \mathcal{V}_{\Omega_{\delta}}$.

- We define $\overline{\mathcal{V}}_{\Omega_{\delta}}:=\mathcal{V}_{\Omega_{\delta}} \cup \partial \mathcal{V}_{\Omega_{\delta}}$.

- We denote by $\Omega_{\delta}^{*}$ the dual graph of $\Omega_{\delta}$, whose vertex set $\mathcal{V}_{\Omega_{\delta}^{*}}$ consists of the midpoints of the bounded faces of $\Omega_{\delta}$ and whose edge set $\mathcal{E}_{\Omega_{\delta}^{*}}$ consists of all pairs of dual vertices of $\mathcal{V}_{\Omega_{\delta}^{*}}$ corresponding to adjacent faces of $\Omega_{\delta}$.

- We denote by $\partial \mathcal{V}_{\Omega_{\delta}^{*}}$ the set of dual vertices of $\mathcal{V}_{\mathbb{C}_{\delta}^{*}} \backslash \mathcal{V}_{\Omega_{\delta}^{*}}$ that are adjacent to a face of $\mathcal{V}_{\Omega_{\delta}^{*}}$, counted with multiplcity: a face of $\mathcal{V}_{\mathbb{C}_{\delta}^{*}} \backslash \mathcal{V}_{\Omega_{\delta}^{*}}$ appear as as many elements of $\partial \mathcal{V}_{\Omega_{\delta}^{*}}$ as there are faces of $\mathcal{V}_{\Omega_{\delta}}$ it is adjacent to.

- We define $\overline{\mathcal{V}}_{\Omega_{\delta}^{*}}:=\mathcal{V}_{\Omega_{\delta}^{*}} \cup \partial \mathcal{V}_{\Omega_{\delta}^{*}}$.

- We denote $\Omega_{\delta}^{m}$ the medial graph, whose vertex set $\mathcal{V}_{\Omega_{\delta}^{m}}$ consists of the midpoints of edges of $\mathcal{E}_{\Omega_{\delta}} \cup \partial \mathcal{E}_{\Omega_{\delta}}$ and whose edge set $\mathcal{E}_{\Omega_{\delta}^{m}}$ consists of all pairs of medial vertices $\mathcal{V}_{\Omega_{\delta}^{m}}$ corresponding to incident edges of $\mathcal{E}_{\Omega_{\delta}} \cup \partial \mathcal{E}_{\Omega_{\delta}}$.

- We denote by $\partial_{0} \mathcal{V}_{\Omega_{\delta}^{m}}$ the set of midpoints of edges of $\partial \mathcal{E}_{\Omega_{\delta}}$. The vertices of $\partial_{0} \mathcal{V}_{\Omega_{\delta}^{m}}$ get naturally identified with prime ends of $\partial \hat{\Omega}_{\delta}$.

- We denote by $\partial_{0} \mathcal{E}_{\Omega_{\delta}^{m}}$ the set of medial edges incident to a medial vertex of $\partial_{0} \mathcal{V}_{\Omega_{\delta}^{m}}$ 
- We denote by $\Omega_{\delta}^{m *}$ the dual of the medial graph, whose vertex set $\mathcal{V}_{\Omega_{\delta}^{m *}}$ is identified with $\mathcal{V}_{\Omega_{\delta}} \cup \mathcal{V}_{\Omega_{\delta}^{*}}$.

- For two boundary medial vertices $v_{1}, v_{2} \in \partial_{0} \mathcal{V}_{\Omega_{\delta}^{m}}$, we denote by $\partial_{0} \mathcal{V}_{\left[v_{1}, v_{2}\right]_{\delta}^{m}} \subset$ $\partial_{0} \mathcal{V}_{\Omega_{\delta}^{m}}$ the set boundary medial vertices identified with prime ends of the counterclockwise arc $\left[v_{1}, v_{2}\right] \subset \partial \hat{\Omega}_{\delta}$. We denote by $\partial_{0} \mathcal{E}_{\left[v_{1}, v_{2}\right]_{\delta}^{m}} \subset \partial_{0} \mathcal{E}_{\Omega_{\delta}^{m}}$ the set of boundary medial edges incident to a vertex of $\partial_{0} \mathcal{V}_{\left[v_{1}, v_{2}\right]_{\delta}^{m}}$.

- With each medial edge $e \in \mathcal{E}_{\Omega_{\delta}^{m}}$, we associate a line $\ell(e) \subset \mathbb{C}$ in the complex plane, defined by $\ell(e):=(\mathfrak{m}-\mathfrak{c})^{-\frac{1}{2}} \mathbb{R}$, where $\mathfrak{m}$ is the midpoint of $e$ and $\mathfrak{c} \in \mathcal{V}_{\Omega_{\delta}}$ is the vertex of $\Omega_{\delta}$ that is the closest to $e$.

- We say that a vertex $v_{1}$ and a medial vertex $v_{2}$ are adjacent if $v_{1}$ is incident to the edge whose midpoint is $v_{2}$.

- For a line $\ell=e^{i \theta} \mathbb{R}$ in the complex plane, we denonte $\mathrm{P}_{\ell}$ the orthgonal projection onto that line, defined by

$$
\mathrm{P}_{\ell}[z]:=\frac{1}{2}\left(z+e^{2 i \theta} \bar{z}\right) \quad \forall z \in \mathbb{C}
$$

- Given two complex numbers $z_{1}, z_{2} \in \mathbb{C}$, we write $z_{1} \| z_{2}$ if $z_{1}$ is a real multiple of $z_{2}$.

- For each boundary medial vertex $z \in \partial_{0} \mathcal{V}_{\Omega_{\delta}^{m}}$, we denote by $\nu_{\text {out }}(z)$ the unit outward-pointing normal of $\mathcal{D}_{\Omega_{\delta}}$ at $z$, i.e. the complex number $\frac{2}{\delta}(z-v)$, where $v \in \mathcal{V}_{\Omega_{\delta}}$ is the vertex incident to the edge $e \in \partial \mathcal{E}_{\Omega_{\delta}^{m}}$ whose midpoint is $z$. We define $\nu_{\text {in }}(z)$ as $-\nu_{\text {out }}(z)$.

- We say that a function $f: \mathcal{V}_{\Omega_{\delta}^{m}} \rightarrow \mathbb{C}$ is s-holomorphic if for each $e=\left\langle v_{1}, v_{2}\right\rangle \in$ $\mathcal{E}_{\Omega_{\delta}^{m}}$, we have

$$
\mathrm{P}_{\ell(e)}\left[f\left(v_{1}\right)\right]=\mathrm{P}_{\ell(e)}\left[f\left(v_{2}\right)\right] .
$$

13.2. Discrete holomorphic observables. We now define the two discrete holomorphic observables that are instrumental in our analysis. These functions are defined on the medial graph of a discrete vertex domain, and their boundary values give the correlation functions appearing in Theorem 29. As one of these observables is more naturally defined in terms of the FK-Ising model and the other in terms of the high-temperature expansion of the spin correlations of the Ising model, we will refer to these as the FK(-Ising) and spin observables.

13.2.1. FK observable. The FK observable was originally introduced in [Smi06] and studied in [Smi10a, ChSm09] to show the convergence of the critical FK-Ising interfaces to SLE(16/3). The key result in this proof is the scaling limit of the observable (see Theorem 81 below). The observable has also proven to be useful to obtain estimates for crossing probabilities [DHN11]. Its boundary values of are of particular interest, as they give the boundary magnetization with mixed $+/$ free boundary conditions (see Section 13.3).

Let $\left(\Omega_{\delta}, r, \ell\right)$ be a discrete domain and consider the FK-Ising model on $\Omega_{\delta}$, with wired boundary condition on $[r, \ell]$ (see Section 8). Let $r_{m}, \ell_{m} \in \partial_{0} \mathcal{V}_{\Omega_{\delta}^{m}}$ be the medial vertices separating $[r, \ell]$ from $\partial \hat{\Omega}_{\delta} \backslash[r, \ell]$ (see Figure 13.2). 


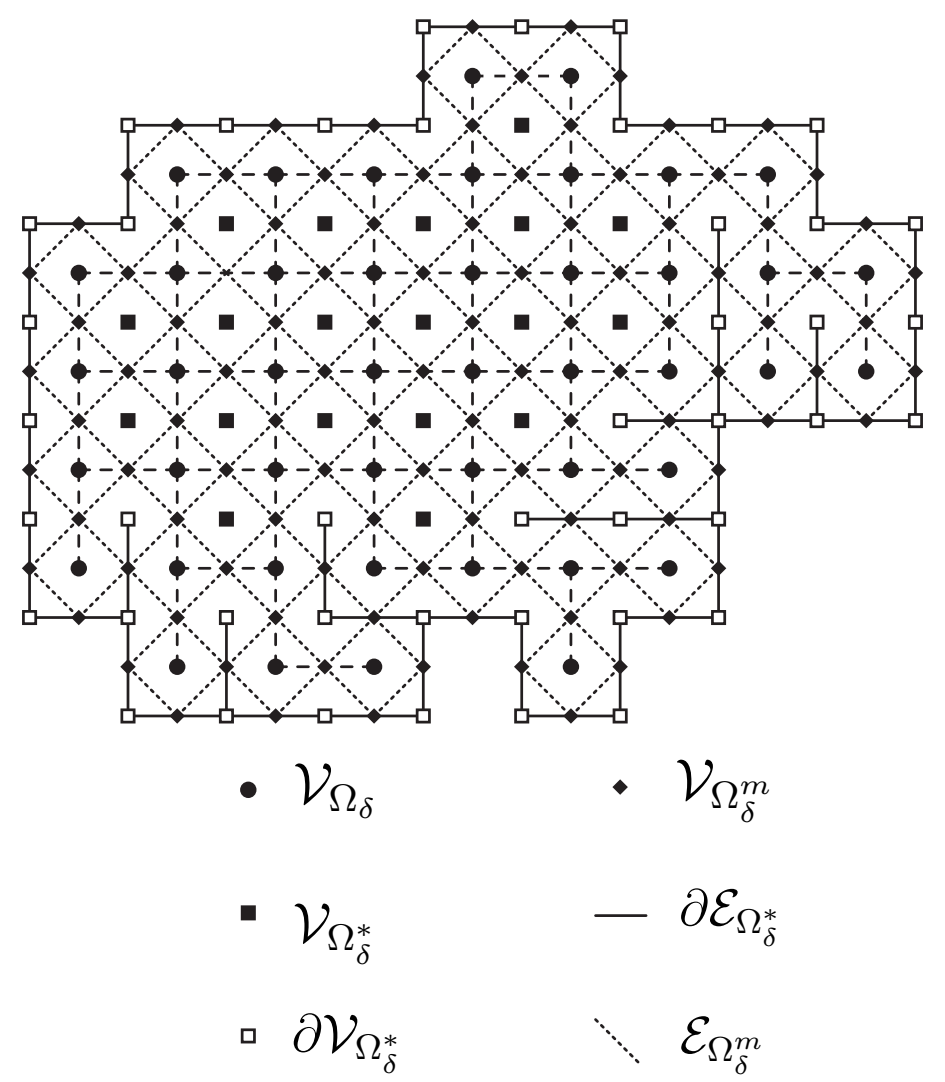

Figure 13.1. Vertex domain, its medial and its dual.

Definition 46. We define the FK-Ising observable $g_{\delta}^{\mathrm{FK}}\left(\Omega_{\delta}, r, \ell, \cdot\right)$ on $\mathcal{E}_{\Omega_{\delta}^{m}} \backslash \partial_{0} \mathcal{E}_{[r, \ell]_{\delta}^{m}}$ by

$$
g_{\delta}^{\mathrm{FK}}\left(\Omega_{\delta}, r, \ell, e\right):=\frac{e^{\frac{\pi i}{4}}}{\sqrt{2}} \cdot \mathbb{E}_{\Omega_{\delta}}^{[r, \ell]_{\mathrm{w}}}\left[\mathbf{1}_{e \in \gamma} e^{-\frac{i}{2} \mathbf{W}\left(\lambda_{\delta}: r_{m} \rightsquigarrow e\right)}\right],
$$

where $\gamma$ is the FK interface linking $r_{m}$ to $\ell_{m}$, rounded as in Figure 13.2, and $\mathbf{W}\left(\lambda_{\delta}: r_{m} \rightsquigarrow e\right)$ is the winding (i.e. the total turning) of the interface $\lambda_{\delta}$ (running backwards) from $r_{m}$ to the midpoint of $e$ (hence we have $\mathbf{W}\left(\lambda_{\delta}: r_{m} \rightsquigarrow e\right) \in$ $\left.\left\{\frac{\pi}{4}+k \frac{\pi}{2}: k \in \mathbb{Z}\right\}\right)$.

Remark 47. The factor $\frac{e^{\pi i / 4}}{\sqrt{2}}$ is introduced in order to follow existing conventions.

Definition 48. We define

$$
f_{\delta}^{\mathrm{FK}}\left(\Omega_{\delta}, r, \ell, e\right):=\frac{1}{\sqrt{\nu_{\mathrm{in}}\left(r_{m}\right)}} g_{\delta}^{\mathrm{FK}}\left(\Omega_{\delta}, r, \ell, e\right),
$$

where we take the following branch of the square root $\sqrt{e^{i \theta}}:=e^{\frac{i \theta}{2}}$ for $\theta \in(-\pi, \pi]$. Remark 49. The branch choice of $\sqrt{\nu_{\text {in }}\left(r_{m}\right)}$ is somewhat arbitrary and is made for definiteness.

Lemma 50. We have

$$
f_{\delta}^{\mathrm{FK}}\left(\Omega_{\delta}, r, \ell, e\right) \in \ell(e) \quad \forall e \in \mathcal{E}_{\Omega_{\delta}^{m}} \backslash \partial_{0} \mathcal{E}_{[r, \ell]_{\delta}^{m}} .
$$




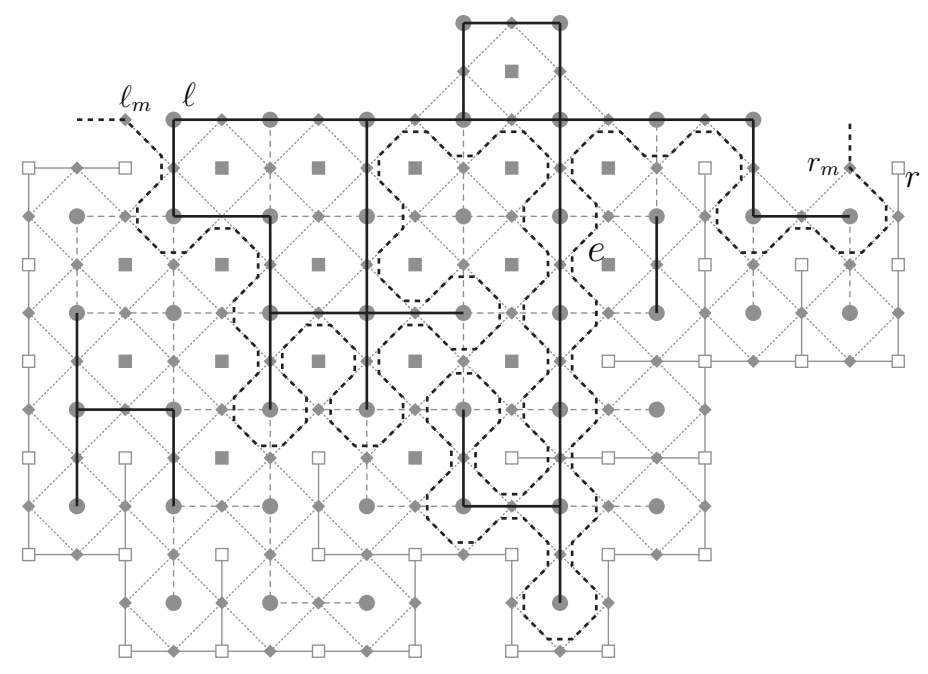

FIgURE 13.2. Setup for the FK observable. On the figure, we have $\mathbf{W}\left(\gamma: r_{m} \rightsquigarrow e\right)=-\frac{\pi}{4}$.

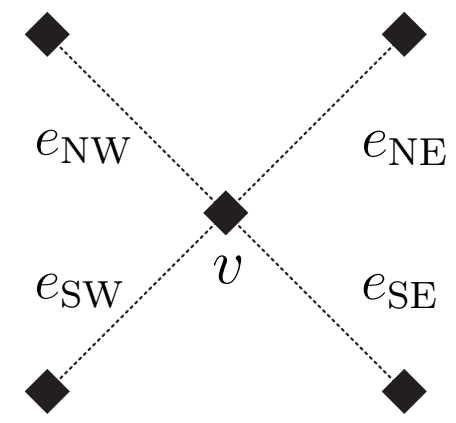

FiguRE 13.3. Medial vertex and edges in Lemma 51 .

Proof. This follows from topological considerations (see [Smi10a, Lemma 4.1]).

Lemma 51. Let $e_{\mathrm{NE}}, e_{\mathrm{NW}}, e_{\mathrm{SW}}, e_{\mathrm{SE}} \in \mathcal{E}_{\Omega_{\delta}^{m}}$ be the four medial edges incident to a medial vertex $v \in \mathcal{V}_{\Omega_{\delta}^{m}} \backslash \partial_{0} \mathcal{V}_{\Omega_{\delta}^{m}}$ as in Figure 13.3. Then we have

$$
f_{\delta}^{\mathrm{FK}}\left(\Omega_{\delta}, r, \ell, e_{\mathrm{NE}}\right)+f_{\delta}^{\mathrm{FK}}\left(\Omega_{\delta}, r, \ell, e_{\mathrm{SW}}\right)=f_{\delta}^{\mathrm{FK}}\left(\Omega_{\delta}, r, \ell, e_{\mathrm{SE}}\right)+f_{\delta}^{\mathrm{FK}}\left(\Omega_{\delta}, r, \ell, e_{\mathrm{NW}}\right) .
$$

Proof. The proof of this is based on combinatorial considerations (see Smi10a, Equation 12]).

This lemma allows us to define $f_{\delta}^{\mathrm{FK}}$ on $\mathcal{V}_{\Omega_{\delta}^{m}} \backslash \partial_{0} \mathcal{V}_{[r, \ell]_{\delta}^{m}}$ in the following way:

Definition 52. For each medial vertex $z \in \mathcal{V}_{\Omega_{\delta}^{m}} \backslash \partial_{0} \mathcal{V}_{[r, \ell]_{\delta}^{m}}$, we define $f_{\delta}^{\mathrm{FK}}\left(\Omega_{\delta}, r, \ell, z\right)$ as the unique complex number such that for each medial edge $e \in \mathcal{E}_{\Omega_{\delta}^{m}} \backslash[r, \ell]_{m}$ that 
is incident to $z$, we have

$$
f_{\delta}^{\mathrm{FK}}\left(\Omega_{\delta}, r, \ell, e\right)=\mathrm{P}_{\ell(e)}\left[f_{\delta}^{\mathrm{FK}}\left(\Omega_{\delta}, r, \ell, z\right)\right] .
$$

Remark 53. For any $z \in \mathcal{V}_{\Omega_{\delta}^{m}}$ having four neighbors in $\mathcal{V}_{\Omega_{\delta}^{m}} \backslash \partial_{0} \mathcal{V}_{[r, \ell]_{\delta}^{m}}$, if we denote by $e_{\mathrm{NE}}, e_{\mathrm{NW}}, e_{\mathrm{SW}}, e_{\mathrm{SE}} \in \mathcal{E}_{\Omega_{\delta}^{m}} \backslash \partial_{0} \mathcal{E}_{[r, \ell]_{\delta}^{m}}$ the medial edges incident to $z$, we have the following orthogonal decompositions:

$$
\begin{aligned}
f_{\delta}^{\mathrm{FK}}\left(\Omega_{\delta}, r, \ell, z\right) & =f_{\delta}^{\mathrm{FK}}\left(\Omega_{\delta}, r, \ell, e_{\mathrm{NE}}\right)+f_{\delta}^{\mathrm{FK}}\left(\Omega_{\delta}, r, \ell, e_{\mathrm{SW}}\right) \\
& =f_{\delta}^{\mathrm{FK}}\left(\Omega_{\delta}, r, \ell, e_{\mathrm{SE}}\right)+f_{\delta}^{\mathrm{FK}}\left(\Omega_{\delta}, r, \ell, e_{\mathrm{NW}}\right) .
\end{aligned}
$$

We will also use a rephased version of the FK observable:

Definition 54. We define $g_{\delta}^{\mathrm{FK}}\left(\Omega_{\delta}, r, \ell, \cdot\right)$ on the medial vertices by

$$
g_{\delta}^{\mathrm{FK}}\left(\Omega_{\delta}, r, \ell, \cdot\right):=\sqrt{\nu_{\mathrm{in}}\left(r_{m}\right)} f_{\delta}^{\mathrm{FK}}\left(\Omega_{\delta}, r, \ell, \cdot\right),
$$

where $\sqrt{\nu_{\text {in }}\left(r_{m}\right)}$ is as in Definition 48 .

The most fundamental analytical property of the FK observable is the following:

Lemma 55. The function $f_{\delta}^{\mathrm{FK}}\left(\Omega_{\delta}, r, \ell, \cdot\right): \mathcal{V}_{\Omega_{\delta}^{m}} \backslash \partial_{0} \mathcal{V}_{[r, \ell]_{\delta}^{m}} \rightarrow \mathbb{C}$ is s-holomorphic.

Proof. This follows directly from the construction of $f_{\delta}^{\mathrm{FK}}$ (Equation 13.3).

Lemma 56. We have

$$
f_{\delta}^{\mathrm{FK}}\left(\Omega_{\delta}, r, \ell, z\right) \in \nu_{\text {out }}^{-\frac{1}{2}}(z) \mathbb{R} \quad \forall z \in \partial_{0} \mathcal{V}_{[\ell, r]_{\delta}^{m}}
$$

Remark 57. Near $\partial_{0} \mathcal{V}_{[r, \ell]_{\delta}^{m}}$, a boundary condition analogous to the one of Lemma 56 holds (see [Smi10a, Lemma 4.12] or [ChSm09, Remark 2.3]), but we will not need to study it for our purposes.

13.2.2. Spin observable. We now define the spin observable, first introduced in [Smi06] and studied in [ChSm09, which is instrumental in the original proof of Chelkak and Smirnov to obtain the convergence of the spin interfaces of the Ising model (with + and - boundary conditions) to chordal SLE(3). A variant of this observable can be used to derive the correlation functions of the energy field of the Ising model [HoSm10b, Hon10a]. Like the FK observable, its boundary values are particularly interesting as they give boundary spin-spin correlations with free boundary conditions (see Section 13.3 or [Hon10a ]).

Let $\Omega_{\delta}$ be a discrete domain. We denote by $\mathcal{Z}\left(\Omega_{\delta}\right)$ the low-temperature expansion of the partition function of the critical Ising model on the faces of $\Omega_{\delta}$, defined by

$$
\mathcal{Z}\left(\Omega_{\delta}\right):=\sum_{\omega \in \mathcal{C}\left(\Omega_{\delta}\right)} \alpha^{|\omega|}
$$

where $\mathcal{C}\left(\Omega_{\delta}\right)$ is the set of contours $\omega \subset \mathcal{E}_{\Omega_{\delta}}$ such that every vertex of $\mathcal{V}_{\Omega_{\delta}}$ is incident to an even number of edges of $\omega$ and where $\alpha:=\sqrt{2}-1$ and $|\omega|$ is the total number of edges of $\omega$.

Let $x \in \partial_{0} \mathcal{V}_{\Omega_{\delta}^{m}}$ be a boundary medial vertex and let $z \in \mathcal{V}_{\Omega_{\delta}^{m}}$ be a medial vertex. We define the collection $\mathcal{C}\left(\Omega_{\delta}, x, z\right)$ as the set of $\gamma$ 's consisting of edges of $\mathcal{E}_{\Omega_{\delta}} \backslash\{z\}$ and of two half-edges (half of an edge, between its midpoint and one of its ends) such that

- one of the half-edges is the unique half-edge incident to $x$. 


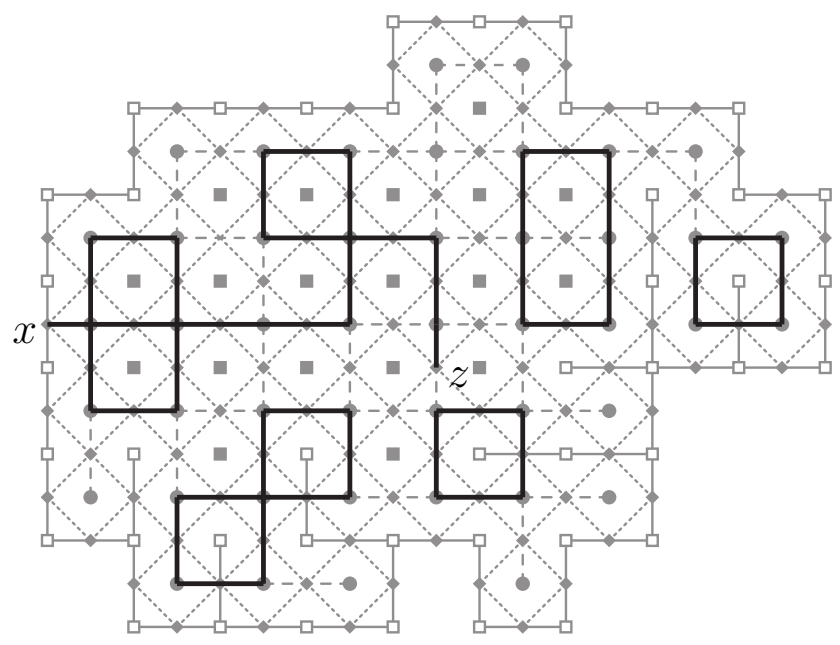

FiguRE 13.4. A configuration $\gamma \in \mathcal{C}_{\Omega_{\delta}}(x, z)$ with three ambiguities and with $\mathbf{W}(\gamma)=-\frac{\pi}{2}$.

- the other half-edge is incident to $z$;

- every vertex $v \in \mathcal{V}_{\Omega_{\delta}}$ belongs to an even number of edges or half-edges of $\gamma$. For a contour $\gamma \in \mathcal{C}\left(\Omega_{\delta}, x, z\right)$, we define its winding $\mathbf{W}(\gamma)$ as the total rotation (the cumulative angle of turn) of the walk on the edges and half-edges of $\gamma$ from $x$ to $z$, which turns left whenever there is an ambiguity (i.e. we arrive at a vertex such that it belongs to four edges or half-edges of $\gamma$ ). See Figure 13.4.

Remark 58. As shown in [HoSm10b, Lemma 4], the complex number $e^{-\frac{i}{2} \mathbf{W}(\gamma)}$ is essentially independent of the choice of the walk on $\gamma$.

Definition 59. We define the spin observable $g_{\delta}^{\text {SPIN }}$ by

$$
g_{\delta}^{\mathrm{SPIN}}\left(\Omega_{\delta}, x, z\right):=\frac{1}{\mathcal{Z}\left(\Omega_{\delta}\right)} \sum_{\gamma \in \mathcal{C}\left(\Omega_{\delta}, x, z\right)} \alpha^{|\gamma|} e^{-\frac{i}{2} \mathbf{W}(\gamma)},
$$

for any $z \in \mathcal{V}_{\Omega_{\delta}^{m}} \backslash\{x\}$, where $|\gamma|$ is the number of edges in $\gamma$, with the two half-edges of $\gamma$ contributing $\frac{1}{2}$ each. We set

$$
g_{\delta}^{\mathrm{SPIN}}\left(\Omega_{\delta}, x, x\right):=1 .
$$

We define $f_{\delta}^{\text {SPIN }}$ by

$$
f_{\delta}^{\mathrm{SPIN}}\left(\Omega_{\delta}, x, z\right):=\frac{1}{\sqrt{\nu_{\text {in }}(x)}} g_{\delta}^{\mathrm{SPIN}}\left(\Omega_{\delta}, x, z\right),
$$

where $\sqrt{\nu_{\text {in }}(x)}$ is the principal determination of the square root as in Definition 46 . Remark 60 . If $z \in \partial_{0} \mathcal{V}_{\Omega_{\delta}^{m}}$, by symmetry, we get

$$
g_{\delta}^{\mathrm{SPIN}}\left(\Omega_{\delta}, x, z\right)=\overline{g_{\delta}^{\mathrm{SPIN}}\left(\Omega_{\delta}, z, x\right)} .
$$

Lemma 61. For any $x \in \partial_{0} \mathcal{V}_{\Omega_{\delta}^{m}}$, the function $f_{\delta}^{\mathrm{SPIN}}\left(\Omega_{\delta}, x, \cdot\right): \mathcal{V}_{\Omega_{\delta}^{m}} \rightarrow \mathbb{C}$ is $s$ holomorphic. 
Proof. This follows from combinatorial considerations. See ChSm09, Proposition 2.5] or [Hon10a, Proposition 74].

Lemma 62. For any $x \in \partial_{0} \mathcal{V}_{\Omega_{\delta}^{m}}$ and all $z \in \partial_{0} \mathcal{V}_{\Omega_{\delta}^{m}} \backslash\{x\}$, we have

$$
f_{\delta}^{\mathrm{SPIN}}\left(\Omega_{\delta}, x, z\right) \in \nu_{\text {out }}^{-\frac{1}{2}}(z) \mathbb{R} .
$$

Proof. This follows from topological considerations. See ChSm09, Equation 2.11] or [Hon10a, Proposition 78].

13.3. Discrete correlation functions. What makes the FK and spin observables particularly relevant to our analysis is that their boundary values give the correlation functions appearing in Theorem 29.

Lemma 63. Let $\left(\Omega_{\delta}, r, \ell\right)$ be a discrete domain. For $z \in \partial_{0} \mathcal{V}_{\Omega_{\delta}^{m}}$ and any boundary medial edge $e \in \partial_{0} \mathcal{E}_{\Omega_{\delta}^{m}}$ incident to $z$, we have

$$
\begin{aligned}
\cos \left(\frac{\pi}{8}\right) \cdot\left|f_{\delta}^{\mathrm{FK}}\left(\Omega_{\delta}, r, \ell, z\right)\right| & =\left|f_{\delta}^{\mathrm{FK}}\left(\Omega_{\delta}, r, \ell, e\right)\right| \\
& =\mathbb{P}_{\Omega_{\delta}}^{[r, \ell]_{\mathrm{w}}}\left\{\lambda_{\delta} \text { separates } z \text { from }[\ell, r]\right\} \\
& =\mathbb{P}_{\Omega_{\delta}}^{[r, \ell]_{\mathrm{w}}}\{z \leftrightarrow[\ell . r]\} \\
& =\mathbb{E}_{\Omega_{\delta}}^{[r, \ell]_{+}}\left[\sigma_{z}\right]
\end{aligned}
$$

Proof. The first identity follows from the definition of $f_{\delta}^{\mathrm{FK}}$ (Equation 13.3) and the boundary condition (see Lemma 56]. For the second identity, see [Smi06, Remark 2.4] or [DHN11, proof of Proposition 5.6]. The remaining identies are derived in Lemma 21 in Section 8.1.

Lemma 64. Let $\left(\Omega_{\delta}, a, z\right)$ be a discrete domain. Let $a_{m} \in \partial_{0} \mathcal{V}_{\Omega_{\delta}^{m}}$ and $z_{m} \in \partial_{0} \mathcal{V}_{\Omega_{\delta}^{m}}$ be boundary medial vertices that are adjacent to $a$ and $z$. Then we have

$$
\frac{1}{\alpha}\left|f_{\delta}^{\mathrm{SPIN}}\left(\Omega_{\delta}, a_{m}, z_{m}\right)\right|=\mathbb{E}_{\Omega_{\delta}}^{\text {free }}\left[\sigma_{a} \sigma_{z}\right] .
$$

where $\alpha=\sqrt{2}-1$.

Proof. This, lemma, which can be found in [Hon10a, Proposition 71], follows from the fact that the winding $\mathbf{W}(\gamma)$ is the same for all $\gamma \in \mathcal{C}_{\Omega}\left(a_{m}, z_{m}\right)$, and from the high-temperature expansion of the spin correlations (the techniques of Proposition 11 can be adapted to get the result). The $\alpha$ denominator comes from the fact that we have to remove the two half-edges incident to $a_{m}$ and $z_{m}$ to get the same contours as in the high-temperature expansions.

13.4. Convolution representation of discrete Riemann BVP. In this subsection we discuss the Riemann-type boundary values taken by the observables. These boundary values, together with the s-holomorphicity, allow for a local representation of the observables in terms of a convolution kernel, which happens to be the spin observable. 
13.4.1. Convolution kernel.

Definition 65. Let $\Omega_{\delta}$ be a discrete domain and $u_{\delta}: \partial_{0} \mathcal{V}_{\Omega_{\delta}^{m}} \rightarrow \mathbb{C}$. We denote by $\mathbb{K}_{\delta}\left[\Omega_{\delta}, u_{\delta}\right]: \mathcal{V}_{\Omega_{\delta}^{m}} \rightarrow \mathbb{C}$ the function defined by

$$
\mathbb{K}_{\delta}\left[\Omega_{\delta}, u_{\delta}\right](z):=\sum_{x \in \partial_{0} \mathcal{V}_{\Omega_{\delta}^{m}}} \mathrm{P}_{\nu_{\mathrm{in}}^{-\frac{1}{2}}(x)}\left[u_{\delta}(x)\right] \cdot g_{\delta}^{\mathrm{SPIN}}\left(\Omega_{\delta}, x, z\right) .
$$

13.4.2. Uniqueness. The kernel $\mathbb{K}_{\delta}$ provides us with a local representation of sholomorphic functions.

Lemma 66. Let $\Omega_{\delta}$ be a discrete domain and let $u_{\delta}: \partial_{0} \mathcal{V}_{\Omega_{\delta}^{m}} \rightarrow \mathbb{C}$ be any given function. Then $\mathbb{K}_{\delta}\left[\Omega_{\delta}, u_{\delta}\right]: \mathcal{V}_{\Omega_{\delta}^{m}} \rightarrow \mathbb{C}$ is the unique s-holomorphic function such that

$$
\left(\mathbb{K}_{\delta}\left[\Omega_{\delta}, u_{\delta}\right]-u_{\delta}\right)(z) \in \nu_{\text {out }}^{-\frac{1}{2}}(z) \mathbb{R} \quad \forall z \in \partial_{0} \mathcal{V}_{\Omega_{\delta}^{m}}
$$

Proof. By Lemma 61, we easily obtain that $\mathbb{K}_{\delta}\left[\Omega_{\delta}, u_{\delta}\right]$ is s-holomorphic, as $\mathrm{P}_{\nu_{\text {in }}^{-\frac{1}{2}}(x)}\left[u_{\delta}(x)\right]$. $g_{\delta}^{\text {SPIN }}\left(\Omega_{\delta}, x, \cdot\right)$ is a real multiple of $f_{\delta}^{\text {SPIN }}\left(\Omega_{\delta}, x, \cdot\right)$ for any $x \in \partial_{0} \mathcal{V}_{\Omega_{\delta}^{m}}$. From Lemma 62. we have that $\mathbb{K}_{\delta}\left[\Omega_{\delta}, u_{\delta}\right]$ satisfies the boundary condition 13.4: for $z \in \partial_{0} \mathcal{V}_{\Omega_{\delta}^{m}}$, notice that we have

$$
\mathrm{P}_{\nu_{\text {in }}^{-\frac{1}{2}}(z)}\left[\mathbb{K}_{\delta}\left[\Omega_{\delta}, u_{\delta}\right](z)\right]=\mathrm{P}_{\nu_{\text {in }}^{-\frac{1}{2}}(z)}\left[u_{\delta}(z)\right]
$$

since $\nu_{\text {in }}^{-\frac{1}{2}}$ and $\nu_{\text {out }}^{-\frac{1}{2}}$ are orthogonal. For the uniqueness, see Hon10a, Proposition 48].

Let us reformulate the above lemma in a form that will be directly useful later.

Lemma 67. Let $\Omega_{\delta}$ be a discrete domain, suppose that $\partial_{0} \mathcal{V}_{\Omega_{\delta}^{m}}=\partial_{0}^{\mathrm{s}} \mathcal{V}_{\Omega_{\delta}^{m}} \cup \partial_{0}^{\mathrm{r}} \mathcal{V}_{\Omega_{\delta}^{m}}$ and let $v_{\delta}: \mathcal{V}_{\Omega_{\delta}^{m}} \rightarrow \mathbb{C}$ be an s-holomorphic function such that

$$
v_{\delta}(z) \in \nu_{\text {out }}^{-\frac{1}{2}}(z) \mathbb{R} \quad \forall z \in \partial_{0}^{\mathrm{r}} \mathcal{V}_{\Omega_{\delta}^{m}} .
$$

Let $u_{\delta}(z): \partial_{0} \mathcal{V}_{\Omega_{\delta}^{m}} \rightarrow \mathbb{C}$ be defined by

$$
u_{\delta}(z)= \begin{cases}\mathrm{P}_{\nu_{\text {in }}^{-\frac{1}{2}}(z)}\left[v_{\delta}(z)\right] & \forall z \in \partial_{0}^{\mathrm{s}} \mathcal{V}_{\Omega_{\delta}^{m}}, \\ 0 & \forall z \in \partial_{0}^{\mathrm{r}} \mathcal{V}_{\Omega_{\delta}^{m}} .\end{cases}
$$

Then we have

$$
v_{\delta}(z)=\mathbb{K}_{\delta}\left[\Omega_{\delta}, u_{\delta}\right](z) \quad \forall z \in \mathcal{V}_{\Omega_{\delta}^{m}} .
$$

\subsection{Continuous holomorphic observables.}

13.5.1. Continuous FK observable. Let us now define the continuous FK observable, following [Smi10a]. By Theorem 81 in Section 13.8.1, it is the scaling limit of the discrete FK observable.

Definition 68. Let $(\Omega, r, \ell)$ be a simply connected domain and let $\varphi_{\Omega}$ be a conformal map $(\Omega, r, \ell) \rightarrow(\mathbb{S},-\infty, \infty)$. We define thesquare of the continuous FK observable $f^{\mathrm{FK}}$ by

$$
\left(f^{\mathrm{FK}}\right)^{2}(\Omega, r, \ell, z):=\frac{1}{i} \cdot \varphi_{\Omega}^{\prime}(z) .
$$


Remark 69. As the conformal map $\varphi_{\Omega}$ is unique up to an additive constant, $\varphi_{\Omega}^{\prime}$ is independent of the choice of $\varphi_{\Omega}$.

Remark 70 . We prefer to define $\left(f^{\mathrm{FK}}\right)^{2}$ rather than $f^{\mathrm{FK}}$ in order to avoid to choose a square root branch.

13.5.2. Continuous Spin observable. To properly define the continuous observable $g^{\mathrm{SPIN}}\left(\Omega_{\delta}, x, z\right)$ as the continuous counterpart of the discrete observable introduced in Section 13.2.2, we need to make an assumption on the regularity of the boundary of $\Omega$ near the point $x$. Notice that the normalization in ChSm09 is different (see Remark 72 below).

Definition 71. Let $(\Omega, x)$ be a simply connected domain, with $x$ being on a smooth part of $\partial \Omega$. Let $\eta_{\Omega}$ be a conformal mapping from $(\Omega, x)$ to $(\mathbb{H}, 0)$. We define the continuous spin observable $g^{\text {SPIN }}$ by

$$
g^{\mathrm{SPIN}}(\Omega, x, z):=\frac{i}{\pi} \sqrt{\eta_{\Omega}^{\prime}(x)} \sqrt{\eta_{\Omega}^{\prime}(z)} \frac{1}{\eta_{\Omega}(z)},
$$

this definition being independent from $\eta_{\Omega}$ and from the branch choice of $\sqrt{\eta_{\Omega}^{\prime}}$.

We define $f^{\text {SPIN }}$ by

$$
f^{\mathrm{SPIN}}(\Omega, x, z):=\frac{1}{\sqrt{\nu_{\text {in }}(x)}} g^{\mathrm{SPIN}}(\Omega, x, z),
$$

where we take the principal branch of the square root (i.e. setting $\sqrt{r e^{i \theta}}:=\sqrt{r} e^{\frac{i \theta}{2}}$, where $\theta$ is chosen in $(-\pi, \pi])$.

Remark 72. In ChSm09, a normalization based on the following observation is used: if $(\Omega, y)$ is a simply connected domain, with $y$ being on a smooth part of $\partial \Omega$ and $x \in \partial \Omega$, we have that

$$
z \mapsto \frac{g^{\operatorname{SPIN}}(\Omega, x, z)}{g^{\operatorname{SPIN}}(\Omega, x, y)}
$$

is well-defined on $\Omega$, even if $g^{\text {SPIN }}(x, \cdot)$ might not be well-defined (the possibly illdefined derivative $\eta_{\Omega}^{\prime}(x)$ in Definition 68 appears in both the numerator and the numerator and hence cancels).

13.6. CFT correlation functions. Let us now give a representation of the CFT correlation functions in terms of the continuous observables:

Lemma 73. Let $(\Theta, y, t),(\tilde{\Theta}, \tilde{y}, \tilde{t})$ and $(\Xi, x, s)$ be domains as in Theorem 29. Then we have

$$
\begin{aligned}
\frac{\left\langle\sigma_{x} \sigma_{s}\right\rangle_{\Xi}^{\text {free }}}{\left\langle\sigma_{x}\right\rangle_{\Theta}^{[y, t]_{+}}} & =\frac{(\sqrt{2}+1)}{\cos \left(\frac{\pi}{8}\right)}\left|\frac{f^{\mathrm{SPIN}}(\Xi, s, x)}{f^{\mathrm{FK}}(\Theta, y, t, x)}\right|, \\
\frac{\left\langle\sigma_{x}\right\rangle_{\tilde{\Theta}}^{\left[\tilde{y}_{\delta}, \tilde{t}_{\delta}\right]_{+}}}{\left\langle\sigma_{x}\right\rangle_{\Theta}^{\left[y_{\delta}, t_{\delta}\right]_{+}}} & =\left|\frac{f^{\mathrm{FK}}(\tilde{\Theta}, \tilde{y}, \tilde{t}, x)}{f^{\mathrm{FK}}(\Theta, y, t, x)}\right| .
\end{aligned}
$$

Proof. This follows from the definitions of the continuous observables $f^{\mathrm{FK}}$ and $f^{\mathrm{SPIN}}$ (Definitions 68 and 71) and of the continuous continuous correlation functions (Definition 13). Notice that all these ratios are well-defined, even when $x$ is on a rough part of the boundary. 
13.7. Convolution representation of continuous Riemann BVP. In this subsection, we discuss the continuous version of the Riemann boundary value problems introduced in Section 13.4. For those, we will only introduce a restricted framework, which is enough for our purposes.

13.7.1. Continuous convolution kernel. Let us first introduce the continuous version of the operator $\mathbb{K}_{\delta}$ introduced in Section 13.4.1.

Definition 74. Let $\Omega$ be a simply connected domain, with $\partial \Omega=\partial^{\mathrm{s}} \Omega \cup \partial^{\mathrm{r}} \Omega$, $\partial^{\mathrm{s}} \Omega$ being compact and piecewise smooth. Let $u: \partial^{s} \Omega \rightarrow \mathbb{C}$ be an arbitrary continuous function. We denote by $\mathbb{K}[\Omega, u]: \Omega \rightarrow \mathbb{C}$ the function defined by

$$
\mathbb{K}[\Omega, u](z):=\int_{\partial^{\mathrm{s}} \Omega} \mathrm{P}_{\nu_{\text {in }}}^{-\frac{1}{2}(x)}[u(x)] \cdot g^{\mathrm{SPIN}}(\Omega, x, z) \mathrm{d}|x| .
$$

13.7.2. Local conformal charts. Let us first define the continuous version of the Riemann-type boundary condition $f(z) \in \nu_{\text {out }}^{-\frac{1}{2}}(z) \mathbb{R}$ discussed in Section 13.4. As the normal vector is not necessarily well-defined anymore, we use local conformal charts.

Definition 75. Let $\Omega$ be a domain and let $f: \Omega \rightarrow \mathbb{C}$ be a holomorphic function. Let $\mathfrak{b} \subset \partial \Omega$ be an arc of the boundary. We say that

$$
f(z) \in \nu_{\text {out }}^{-\frac{1}{2}}(z) \mathbb{R} \text { on } \mathfrak{b}
$$

if there is a simply connected domain $\Upsilon$ coinciding with $\Omega$ in a neighborhood of $\mathfrak{b}$ and a point $a$ on a smooth part of $\partial \Upsilon \backslash \partial \Omega$ such that for any $p \in \mathfrak{b}$, we have that

$$
\lim _{z \rightarrow p} \frac{f(z)}{f^{\operatorname{SPIN}}(\Upsilon, a, z)} \in \mathbb{R} .
$$

Remark 76. When $\partial \Omega$ is smooth, this boundary condition equivalent to having $f$ extending continuously to $\mathfrak{b}$ and satisfying $f(z) \in \nu_{\text {out }}^{-\frac{1}{2}}(z) \mathbb{R}$ for each $z \in \mathfrak{b}$.

Thanks to the following lemma, the above definition does not depend on the choice of $\Upsilon$ or of $a$.

Lemma 77. If there exists such a domain $\Upsilon$ and a point $a \in \partial \Upsilon$ on a smooth part of $\partial \Upsilon \backslash \partial \Omega$, then the for all $\tilde{\Upsilon}$ coinciding with $\Omega$ in neighborhood of $\mathfrak{b}$ and any $\tilde{a} \in \partial \tilde{\Upsilon}$ on a smooth part of $\partial \tilde{\Upsilon} \backslash \partial \Omega$, the condition of Equation 13.5 is satisfied.

Proof. It is enough to prove that for any $p \in \mathfrak{b}$, we have

$$
\lim _{z \rightarrow p} \frac{f^{\operatorname{SPIN}}(\Upsilon, a, z)}{f^{\operatorname{SPIN}}(\tilde{\Upsilon}, \tilde{a}, z)} \in \mathbb{R}
$$

It is enough to prove 13.6 in the following two cases:

- When $a=\tilde{a}$ and $\Upsilon, \tilde{\Upsilon}$ moreover coincide in a neighborhood of $a$, to prove 13.6. we can assume that $\tilde{\Upsilon} \subset \Upsilon$ (otherwise replace $\tilde{\Upsilon}$ by $\Upsilon \cap \tilde{\Upsilon}$ ). Let $\psi_{\Upsilon}: \Upsilon \rightarrow \mathbf{D}(0,1)$ be a conformal map. Setting $\tilde{\mathbf{D}}:=\psi_{\Upsilon}(\tilde{\Upsilon}) \subset \mathbf{D}(0,1)$, 
by conformal covariance of $g^{\text {SPIN }}$ (which follows from its definition), we have (noticing that the derivative terms cancel):

$$
\frac{f^{\mathrm{SPIN}}(\Upsilon, a, z)}{f^{\mathrm{SPIN}}(\tilde{\Upsilon}, \tilde{a}, z)}=\frac{g^{\mathrm{SPIN}}(\Upsilon, a, z)}{g^{\mathrm{SPIN}}(\tilde{\Upsilon}, \tilde{a}, z)}=\frac{g^{\mathrm{SPIN}}\left(\mathbf{D}(0,1), \psi_{\Upsilon}(a), \psi_{\Upsilon}(z)\right)}{g^{\mathrm{SPIN}}\left(\tilde{\mathbf{D}}, \psi_{\Upsilon}(a), \psi_{\Upsilon}(z)\right)} .
$$

As $z \rightarrow p, \psi_{\Upsilon}(z) \rightarrow \partial \mathbf{D}(0,1) \cap \partial \tilde{\mathbf{D}}$, and as $\partial \mathbf{D}(0,1)$ is smooth, it is easy to check that the right-hand side tends to a purely real number.

- When $\Upsilon=\tilde{\Upsilon}$, taking a conformal map $\psi_{\Upsilon}: \Upsilon \rightarrow \mathbf{D}(0,1)$, by conformal covariance of $g^{\text {SPIN }}$, we get

$$
\begin{aligned}
\frac{f^{\operatorname{SPIN}}(\Upsilon, a, z)}{f^{\operatorname{SPIN}}(\Upsilon, \tilde{a}, z)} & =\frac{\sqrt{\nu_{\text {in }}(\tilde{a})}}{\sqrt{\nu_{\text {in }}(a)}} \frac{g^{\text {SPIN }}}{g^{\operatorname{SPIN}}(\Upsilon, a, z)} \\
& =\frac{\sqrt{\nu_{\text {in }}\left(\psi_{\Upsilon}(\tilde{a})\right)}}{\sqrt{\nu_{\text {in }}\left(\psi_{\Upsilon}(a)\right)}} \frac{\sqrt{\left|\psi_{\Upsilon}^{\prime}(a)\right|}}{\sqrt{\left|\psi_{\Upsilon}^{\prime}(\tilde{a})\right|}} \frac{g^{\operatorname{SPIN}}\left(\mathbf{D}(0,1), \psi_{\Upsilon}(a), \psi_{\Upsilon}(z)\right)}{g^{\operatorname{SPIN}}\left(\mathbf{D}(0,1), \psi_{\Upsilon}(\tilde{a}), \psi_{\Upsilon}(z)\right)} \\
& = \pm \frac{\sqrt{\left|\psi_{\Upsilon}^{\prime}(a)\right|}}{\sqrt{\left|\psi_{\Upsilon}^{\prime}(\tilde{a})\right|}} \frac{f^{\operatorname{SPIN}}\left(\mathbf{D}(0,1), \psi_{\Upsilon}(a), \psi_{\Upsilon}(z)\right)}{f^{\operatorname{SPIN}}\left(\mathbf{D}(0,1), \psi_{\Upsilon}(\tilde{a}), \psi_{\Upsilon}(z)\right)},
\end{aligned}
$$

where the \pm sign comes the branch of the square root. Since $\partial \mathbf{D}(0,1)$ is smooth, it is easy to check that the the right-hand side tends to a purely real number as $z \rightarrow p$.

Lemma 78. Let $\left(\Omega^{(1)}, r, \ell\right)$ be a simply connected domain. The function $f^{\mathrm{FK}}\left(\Omega^{(1)}, r, \ell, \cdot\right)$ satisfies the boundary condition

$$
f^{\mathrm{FK}}\left(\Omega^{(1)}, r, \ell, z\right) \in \nu_{\mathrm{out}}^{-\frac{1}{2}}(z) \mathbb{R} \text { on the compact subsets of }[\ell, r] \backslash\{\ell, r\} .
$$

Let $\left(\Omega^{(2)}, x\right)$ be a simply connected domain such that $x$ is on a smooth part of $\partial \Omega^{(2)}$. The function $f^{\mathrm{SPIN}}\left(\Omega^{(2)}, x, \cdot\right)$ satifies the boundary condition

$$
f^{\text {SPIN }}\left(\Omega^{(2)}, x, z\right) \in \nu_{\text {out }}^{-\frac{1}{2}}(z) \mathbb{R} \text { on the compact subsets of } \partial \Omega^{(2)} \backslash\{x\} .
$$

Proof. The proof is essentially the same as the one of Lemma 77, we obtain a representation of the observables in terms of the same conformal map, and the possibly ill-defined derivative terms appearing in the numerator and the denominator of the fractions cancel.

13.7.3. Convolution representation and uniqueness. We now give the lemma which provides us with a local representation of functions satisfying Riemann-type boundary conditions in terms of the convolution kernel $\mathbb{K}$.

Lemma 79. Let $\Omega$ be a simply connected domain with $\partial \Omega=\partial^{\mathrm{s}} \Omega \cup \partial^{\mathrm{r}} \Omega$, $\partial^{\mathrm{s}} \Omega$ being compact and piecewise smooth. Let $u: \partial^{s} \Omega \rightarrow \mathbb{C}$ be an arbitrary continuous function. Then the function $\mathbb{K}[\Omega, u]: \Omega \rightarrow \mathbb{C}$ is the unique holomorphic function satisfying

$$
\begin{aligned}
\mathbb{K}[\Omega, u](z)-u(z) & \in \nu_{\text {out }}^{-\frac{1}{2}}(z) \forall z \in \partial^{\mathrm{s}} \Omega \\
\mathbb{K}[\Omega, u](z) & \in \nu_{\text {out }}^{-\frac{1}{2}}(z) \text { on } \partial^{\mathrm{r}} \Omega
\end{aligned}
$$


Proof. We have that $\mathbb{K}[\Omega, u]$ is a real convolution of $f^{\text {SPIN }}$. It follows from Lemma 78 that $\mathbb{K}[\Omega, z]$ satisfies the boundary condition 13.7 . Now, for the uniqueness, suppose there are two holomorphic functions solving this problem and denote by $f$ their difference. Fix $a \in \partial^{\mathrm{s}} \Omega$. We have that

$$
z \mapsto \frac{f(z)}{f^{\operatorname{SPIN}}(\Omega, a, z)}
$$

extends continuously to $z=a$ (where it is equal to 0 , as $\left|f^{\operatorname{SPIN}}(\Omega, a, z)\right| \rightarrow \infty$ when $z \rightarrow a$ ) and that its imaginary part tends to 0 as $z \rightarrow \partial \Omega$. Hence this function is identically equal to 0 .

13.7.4. Well-definedness of ratios on the boundary. Thanks to Lemmas 78 and 79 above, we get a convenient convolution representation of the observables introduced in Section 13.5 in a neighborhood of the boundary. We can now access the boundary values of these observables taking ratios with a given reference observable (it is convenient to choose the spin observable for our purposes).

Lemma 80. Let $\Omega$ be a simply connected domain with $\partial \Omega=\partial^{\mathrm{s}} \Omega \cup \partial^{\mathrm{r}} \Omega$, $\partial^{\mathrm{s}} \Omega$ being compact and piecewise smooth. Let $u: \partial^{\mathrm{s}} \Omega \rightarrow \mathbb{C}$ be a continuous function and let $x \in \partial^{\mathrm{s}} \Omega$ be on a smooth part of $\partial^{\mathrm{s}} \Omega$. Then the ratio

$$
z \mapsto \frac{\mathbb{K}[\Omega, u](z)}{f^{\operatorname{SPIN}}(\Omega, x, z)}
$$

extends continuously to $\partial^{\mathrm{r}} \Omega$ and is purely real there. This ratio varies continuously with respect to $u$.

The ratio 13.8 is is also Carathéodory-stable with respect to perturbation of $\partial^{\mathrm{r}} \Omega$ : fix a smooth curve $\gamma$, a continuous function $u: \gamma \rightarrow \mathbb{C}$, a compact set $\mathcal{K}$ such that $\gamma \subset \partial \mathcal{K}, x \in \gamma$ and $w \in \operatorname{Int}(\mathcal{K})$; for any $\epsilon>0$, there exists $\mu>0$ such that if $\Omega^{(1)}$ and $\Omega^{(2)}$ are two domains such that $\mathcal{K} \subset \Omega_{1} \cap \Omega_{2}$ and $\gamma \subset \partial \Omega_{1} \cap \partial \Omega_{2}$ that are $\mu$-close in Carathéodory metric with respect to $w$, then

$$
\left|\frac{\mathbb{K}\left[\Omega^{(1)}, u\right](z)}{f^{\operatorname{SPIN}}\left(\Omega^{(1)}, x, z\right)}-\frac{\mathbb{K}\left[\Omega^{(2)}, u\right](z)}{f^{\operatorname{SPIN}}\left(\Omega^{(2)}, x, z\right)}\right| \leq \epsilon \quad \forall z \in \mathcal{K} .
$$

Proof. This follows from Lemma 78. The Carathéodory-stability follows from the definitions of the observables in terms of conformal mappings.

13.8. Convergence of observables. In this subsection, we discuss and adapt some results of [Smi10a, ChSm09, HoSm10b, Hon10a] to get the convergence of the discrete observables to their continuous counterparts.

13.8.1. Scaling limit of FK observable. Let us now state the important result concerning scaling limit of the FK observable [Smi10a]. In [Smi06], it is the key result allowing for the proof of Theorem 31 .

Theorem 81. Let $d, D>0$. Let $\left(\Omega_{\delta}, r, \ell\right)$ be a discrete domain with diam $\left(\Omega_{\delta}\right) \leq D$. Then for any $\epsilon>0$, there exists $\delta_{0}>0$ function of $d, D$ only such that for any $\delta \leq \delta_{0}$ and any $z \in \mathcal{V}_{\Omega_{\delta}^{m}}$ with $\operatorname{dist}\left(z, \partial \Omega_{\delta}\right) \geq d$, we have

$$
\left|\frac{1}{\delta}\left(f_{\delta}^{\mathrm{FK}}\right)^{2}\left(\Omega_{\delta}, r, \ell, z\right)-\left(f^{\mathrm{FK}}\right)^{2}(\Omega, r, \ell, z)\right| \leq \epsilon
$$


Proof. This is the main result of [Smi10a, Theorem 2.2]. It is generalized in [ChSm09, Theorem 4.3] in a form closer to the form that we use here. Remark that the normalization is slightly different there: the mesh size $\delta$ in our paper corresponds to $\sqrt{2} \delta$ with the notation of Smi10a.

13.8.2. Scaling limit of spin observable. To obtain the scaling limit of the spin observable, we merge two existing results: in [Hon10a, the convergence of the observable is derived, with the additional assumption that the boundary is piecewise smooth, while in [ChSm09], the convergence of is derived for general domains, but with a different normalization (see Remark 72 above).

Let us first specialize a result of [Hon10a] to the case of a straight domain, i.e. a polygonal domain with horizontal and vertical sides only. Such a domain will serve us as reference domain. This result will be used in the proof of Theorem 84, both to get precompactness of the spin observable on general domains and to identify the limit.

Lemma 82. Let $\mathbf{Q}$ be a straight domain. Let $a \in \partial \mathbf{Q}$ be at the midpoint of a side. For each $\delta>0$, denote by $\mathbf{Q}_{\delta}$ the discrete domain defined by $\mathbf{Q}_{\delta}:=\mathbf{Q} \cap \delta \mathbb{Z}^{2}$ and by $a_{\delta} \in \partial_{0} \mathcal{V}_{\mathbf{Q}_{\delta}}$ boundary medial vertex that is the closest to a and let $\left\{c_{\delta}^{1}, \ldots, c_{\delta}^{m}\right\}$ be the corners of $\mathbf{Q}_{\delta}$. Let $d>0$. Then for each $\epsilon>0$, there exists $\delta_{0}>0$ such that for any $\delta \leq \delta_{0}$, we have

$$
\begin{aligned}
& \quad\left|\frac{1}{\delta} f_{\delta}^{\mathrm{SPIN}}\left(\mathbf{Q}_{\delta}, a_{\delta}, z_{\delta}\right)-f^{\mathrm{SPIN}}\left(\mathbf{Q}_{\delta}, a_{\delta}, z_{\delta}\right)\right| \leq \epsilon \\
& \forall z_{\delta} \in \mathcal{V}_{\mathbf{Q}_{\delta}}: \operatorname{dist}\left(z_{\delta},\left\{a_{\delta}, c_{\delta}^{1}, \ldots, c_{\delta}^{m}\right\}\right) \geq d .
\end{aligned}
$$

Proof. Suppose for definiteness that $a$ is the midpoint of the left side of $\mathbf{Q}$. We can apply Theorem 90 in Hon10a, which holds for piecewise smooth domains. The function $f_{\delta}^{\text {SPIN }}\left(\mathbf{Q}_{\delta}, a_{\delta}, z_{\delta}\right)$ corresponds, in the notation of [Hon10a] to the function $h_{\mathbf{S}_{\delta}}\left(a_{\delta}^{(1)^{2}}, z_{\delta}^{(\sqrt{o})^{2}}\right)+h_{\mathbf{S}_{\delta}}\left(a_{\delta}^{(1)^{2}}, z_{\delta}^{-(\sqrt{o})^{2}}\right)$, where $\sqrt{o}=1$ if $z_{\delta}$ the midpoint of a horizontal edge and $\sqrt{o}=e^{i \pi / 4}$ if it is the midpoint of a vertical one. From [Hon10a, Theorem 90], we obtain the convergence of $f_{\delta}^{\mathrm{SPIN}}\left(\mathbf{Q}_{\delta}, a_{\delta}, \cdot\right)$ to $f^{\mathrm{SPIN}}(\mathbf{Q}, a, \cdot)$. To obtain the lemma, notice that $f^{\mathrm{SPIN}}(\mathbf{Q}, a, \cdot)$ and $f^{\mathrm{SPIN}}\left(\mathbf{Q}_{\delta}, a_{\delta}, \cdot\right)$ are uniformly close (see Lemma 80 above).

The next result that we need is the convergence of ratios of the spin observable in arbitrary domains, obtained in [ChSm09].

Theorem 83. Let $\varrho, D>0$. For each $\delta>0$, let $\left(\Omega_{\delta}, a_{\delta}, z_{\delta}\right)$ be a discrete domain with $\partial \Omega_{\delta}=\mathfrak{s}_{\delta} \cup \mathfrak{r}_{\delta}$ such that $\mathfrak{s}_{\delta}$ is made of a finite number of horizontal and vertical segments $\left\{\left[p_{\delta}^{j}, q_{\delta}^{j}\right]: j=1, \ldots, n\right\}$, diam $\left(\left[p_{\delta}^{j}, q_{\delta}^{j}\right]\right) \geq \varrho$ for each $j \in\{1, \ldots, n\}, a_{\delta} \in$ $\mathfrak{r}_{\delta}$, dist $\left(a_{\delta}, \mathfrak{s}_{\delta}\right) \geq \varrho$ and $\operatorname{diam}\left(\Omega_{\delta}\right) \leq D$. Then for any $d>0$ and any $\epsilon>0$, there exists $\delta_{0}>0$ (function of $d, D, \epsilon$ only) such that for any $\delta \leq \delta_{0}$, we have

for any $z_{\delta} \in \mathcal{V}_{\Omega_{\delta}}$ such that

$$
\left|\frac{f_{\delta}^{\text {SPIN }}\left(\Omega_{\delta}, a_{\delta}, z_{\delta}\right)}{f_{\delta}^{\text {SPIN }}\left(\Omega_{\delta}, a_{\delta}, y_{\delta}\right)}-\frac{f^{\text {SPIN }}\left(\Omega_{\delta}, a_{\delta}, z_{\delta}\right)}{f^{\text {SPIN }}\left(\Omega_{\delta}, a_{\delta}, y_{\delta}\right)}\right| \leq \epsilon
$$

$$
\operatorname{dist}\left(z_{\delta}, \mathfrak{r}_{\delta} \cup \bigcup_{j=1}^{n}\left\{p_{\delta}^{j}, q_{\delta}^{j}\right\}\right) \geq d .
$$




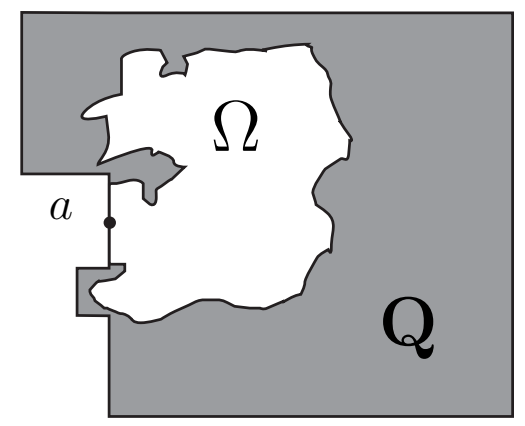

FiguRE 13.5. The domain $\Omega$ and the straight domain $\mathbf{Q}$.

Proof. This follows from Theorem 5.9 and Corollary 5.10 in [ChSm09.

Thanks to Lemma 82 and Theorem 83 , we can derive the following convergence theorem for the spin observable when $a$ is on a straight part of the boundary:

Theorem 84. Let $d, D>0$. For each $\delta>0$, let $\left(\Omega_{\delta}, p_{\delta}, a_{\delta}, q_{\delta}\right)$ be a discrete domain such that $\left[p_{\delta}, q_{\delta}\right]$ is either horizontal or vertical with $\operatorname{dist}\left(p_{\delta}, q_{\delta}\right) \geq d$, dist $\left(a_{\delta},\left[q_{\delta}, p_{\delta}\right]\right) \geq$ $d$ and with $\operatorname{diam}\left(\Omega_{\delta}\right) \leq D$. Then for any $\epsilon>0$, there exists $\delta_{0}>0$ (function of $d, D, \epsilon$ only) such that for $\delta \leq \delta_{0}$

$$
\begin{aligned}
& \left|\frac{1}{\delta} f_{\delta}^{\mathrm{SPIN}}\left(\Omega_{\delta}, a_{\delta}, z_{\delta}\right)-f^{\mathrm{SPIN}}\left(\Omega_{\delta}, a_{\delta}, z_{\delta}\right)\right| \leq \epsilon \\
& \left|\frac{1}{\delta} g_{\delta}^{\mathrm{SPIN}}\left(\Omega_{\delta}, a_{\delta}, z_{\delta}\right)-g^{\mathrm{SPIN}}\left(\Omega_{\delta}, a_{\delta}, z_{\delta}\right)\right| \leq \epsilon
\end{aligned}
$$

for all $z_{\delta} \in \mathcal{V}_{\Omega_{\delta}^{m}}$ with $\operatorname{dist}\left(z_{\delta}, \partial \Omega_{\delta}\right) \geq d$.

Proof. The statements for $f^{\text {SPIN }}$ and $g^{\text {SPIN }}$ are obviously equivalent. Suppose for definiteness that $\left[p_{\delta}, q_{\delta}\right]$ is vertical and that the domain $\Omega_{\delta}$ lies on the right of $\left[p_{\delta}, q_{\delta}\right]$. Set $f_{\delta}:=f_{\delta}^{\text {SPIN }}=g_{\delta}^{\text {SPIN }}$ and $f:=f^{\text {SPIN }}=g^{\text {SPIN }}$. Take a straight domain $\mathbf{Q}$ as in Lemma 82 containing $\Omega$ (see Figure 13.5), with $a$ being the midpoint of one of its sides, and denote by $\mathbf{Q}_{\delta}$ its discretizations as in Lemma 82 , aligned in such a way that the $\left[p_{\delta}, q_{\delta}\right] \subset \partial \mathbf{Q}_{\delta}$ and that the points $a_{\delta}$ of $\partial_{0} \mathcal{V}_{\mathbf{Q}_{\delta}^{m}}$ and $\partial_{0} \mathcal{V}_{\Omega_{\delta}^{m}}$ coincide.

To prove the result, we proceed by contradiction, as in the proof of [ChSm09], Theorem 5.9. Suppose that we can find an $\epsilon>0$ and a sequence $\left(\Omega_{\delta_{n}}, a_{\delta_{n}}\right)$ of discrete domains of mesh size $\delta_{n} \rightarrow 0$ satisfying the assumptions of the theorem and a sequence of points $z_{\delta_{n}} \rightarrow z$ such that the conclusion fails. For each $n \geq 0$, we can moreover choose a point $y_{\delta_{n}}$ such that $y_{\delta_{n}} \in\left[a_{\delta_{n}}, q_{\delta_{n}}\right]$ such that $\operatorname{dist}\left(y_{\delta_{n}}, a_{\delta_{n}}\right) \geq \frac{1}{3} d$ and dist $\left(y_{\delta_{n}},\left[q_{\delta_{n}}, p_{\delta_{n}}\right]\right) \geq \frac{1}{3} d$.

(1) Notice first that the sequence of discrete domains $\left(\Omega_{\delta_{n}}, p_{\delta_{n}}, a_{\delta_{n}}, y_{\delta_{n}}, q_{\delta_{n}}\right)_{n>0}$ is precompact in Carathéodory topology with respect to $z$ and hence that there is a continuous domain $(\Omega, p, a, y, q)$ such that (a susbsequence of) this sequence converges to $(\Omega, p, a, y, q)$.

(2) Precompactness: we show that the family of functions is uniformly bounded 
(a) We have $\left|f_{\delta_{k}}\left(\Omega_{\delta_{k}}, a_{\delta_{k}}, y_{\delta_{k}}\right)\right| \leq\left|f_{\delta_{k}}\left(\mathbf{Q}_{\delta_{k}}, a_{\delta_{k}}, y_{\delta_{k}}\right)\right|$, by Lemma 64, as we have

$$
\begin{aligned}
\left|f_{\delta_{k}}\left(\Omega_{\delta_{k}}, a_{\delta_{k}}, y_{\delta_{k}}\right)\right| & =(\sqrt{2}-1) \mathbb{E}_{\Omega_{\delta_{k}}}^{\text {free }}\left[\sigma\left(a_{\delta_{k}}\right) \sigma\left(y_{\delta_{k}}\right)\right] \\
& \leq(\sqrt{2}-1) \mathbb{E}_{\mathbf{Q}_{\delta_{k}}}^{\text {free }}\left[\sigma\left(a_{\delta_{k}}\right) \sigma\left(y_{\delta_{k}}\right)\right] \\
& =\left|f_{\delta_{k}}\left(\mathbf{Q}_{\delta_{k}}, a_{\delta_{k}}, y_{\delta_{k}}\right)\right|
\end{aligned}
$$

where we used on the second line that $\Omega_{\delta_{k}} \subset \mathbf{Q}_{\delta_{k}}$ and that two-spin correlations with free boundary conditions are monotone increasing with respect to the domains (this follows from FKG inequality, see Gri06).

(b) Hence $\frac{1}{\delta_{k}} f_{\delta_{k}}\left(\Omega_{\delta_{k}}, a_{\delta_{k}}, y_{\delta_{k}}\right)$ is uniformly bounded as $\frac{1}{\delta_{k}} f_{\delta_{k}}\left(\mathbf{Q}_{\delta_{k}}, a_{\delta_{k}}, y_{\delta_{k}}\right)$ is uniformly bounded by Lemma 82 , being uniformly convergent.

(c) By Theorem 83 and Lemma 80 , we have that

$$
\frac{f_{\delta_{k}}\left(\Omega_{\delta_{k}}, a_{\delta_{k}}, \cdot\right)}{f_{\delta_{k}}\left(\Omega_{\delta_{k}}, a_{\delta_{k}}, y_{\delta_{k}}\right)}
$$

is uniformly convergent on every compact set of $\bar{\Omega} \backslash([q, p] \cup\{a\})$, in the sense that for each compact set $K \subset \bar{\Omega} \backslash([q, p] \cup\{a\})$, the restriction of 13.9 to $K \cap \mathcal{V}_{\Omega_{\delta_{k}}}$ is uniformly convergent. Hence, by the previous point, we deduce that the family $\frac{1}{\delta_{k}} f_{\delta_{k}}\left(\Omega_{\delta_{k}}, a_{\delta_{k}}, \cdot\right)$ is precompact for the topology of uniform convergence on the compact subsets of $\bar{\Omega} \backslash$ $([q, p] \cup\{a\})$.

(d) By extracting once more a subsequence, we can suppose that

$$
\frac{1}{\delta_{k}} f_{\delta_{k}}\left(\Omega_{\delta_{k}}, a_{\delta_{k}}, \cdot\right)
$$

is uniformly convergent on the compact subsets of $\bar{\Omega} \backslash([q, p] \cup\{a\})$. Denote by $\tilde{f}$ this limit.

(3) Identification of the limit. Let us now show that $\tilde{f}(\cdot)=f(\Omega, a, \cdot)$. We show the following two properties: both functions have the same boundary conditions and the same pole at $a$ and this characterizes them uniquely (to check this, take the difference of two functions satisfying these properties and get that it is equal to 0 using Lemma 79.

(a) The function $\tilde{f}$ satisfies the boundary condition $\tilde{f}(z) \in \nu_{\text {out }}^{-\frac{1}{2}}(z) \mathbb{R}$ on $\partial \Omega \backslash\{a\}$ : this follows directly from [ChSm09, Theorem 5.9] as

$$
\frac{f_{\delta_{k}}\left(\Omega_{\delta_{k}}, a_{\delta_{k}}, \cdot\right)}{f_{\delta_{k}}\left(\Omega_{\delta_{k}}, a_{\delta_{k}}, y_{\delta_{k}}\right)} \rightarrow \frac{f(\Omega, a, \cdot)}{f(\Omega, a, y)},
$$

and $f(\Omega, a, y) \in \mathbb{R}$.

(b) The function $v:=\tilde{f}(\cdot)-f(\mathbf{Q}, a, \cdot)$ is uniformly bounded in a neighborhood of $a$ : take indeed a small rectangle $R_{\delta_{k}} \subset \Omega_{\delta_{k}}$ such that $\partial R_{\delta_{k}}=\partial_{\delta_{k}}^{1} \cup \partial_{\delta_{k}}^{2}$ with $\partial_{\delta_{k}}^{1} \subset \Omega_{\delta_{k}}$ and $a_{\delta_{k}} \in \partial_{\delta_{k}}^{2} \subset\left[p_{\delta_{k}}, q_{\delta_{k}}\right]$ (see Figure 13.6. . On $\partial_{\delta_{k}}^{1}$, we have that $v_{\delta_{k}}:=\frac{1}{\delta_{k}}\left(f_{\delta_{k}}\left(\Omega_{\delta_{k}}, a_{\delta_{k}}, \cdot\right)-f_{\delta_{k}}\left(\mathbf{Q}_{\delta_{k}}, a, \cdot\right)\right)$ is uniformly bounded. On $\partial_{\delta_{k}}^{2}$, we have the boundary condition

$$
v_{\delta_{k}}(z) \in \nu_{\text {out }}^{-\frac{1}{2}}(z) \mathbb{R} \quad \forall z \in \partial_{\delta_{k}}^{2}
$$




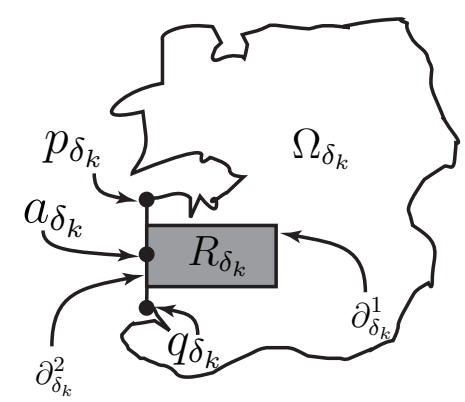

FiguRE 13.6. The rectangle $R_{\delta_{k}} \subset \Omega_{\delta_{k}}$.

as $v_{\delta_{k}}\left(a_{\delta_{k}}\right)=0$. We then have that the restriction of $v_{\delta_{k}}$ to $R_{\delta_{k}}$ is equal to

$$
\mathbb{K}_{\delta_{k}}\left[R_{\delta_{k}},\left.g_{\delta_{k}}\right|_{\partial_{\delta_{k}}^{1}}\right]
$$

Hence, it follows easily from the representation of $\mathbb{K}_{\delta_{k}}$ (the integrands appearing in it being uniformly bounded) that $v_{\delta_{k}}$ is uniformly bounded near $a$. And hence $\lim _{k \rightarrow \infty} v_{\delta_{k}}=\tilde{f}(\cdot)-f(\mathbf{Q}, a, \cdot)$ is also uniformly bounded.

Finally, using Lemma 80 , we obtain $\frac{1}{\delta_{k}} f_{\delta_{k}}\left(\Omega_{\delta_{k}}, a_{\delta_{k}}, z_{\delta_{k}}\right) \rightarrow f(\Omega, a, z)$ as $k \rightarrow \infty$, which contradicts the definition of $z_{\delta_{k}}$.

13.9. Proof of Theorem 29. In this subsection, we prove the main convergence theorem of Section 13 (Theorem 29). The central idea is to localize the convergence results of Theorems 81 and 83 on the boundary, by representing them in terms of the convolution kernel introduced in Section 13.4.1.

Let us first introduce some notation. Recall that $\Xi, \Theta$ and $\tilde{\Theta}$ are domains coinciding in a neighborhood of a boundary point $x$.

Definition 85. Let $\Xi_{\delta}$ be a discrete vertex domain. Let $x_{\delta} \in \partial \Xi_{\delta}$ and let $s_{\delta} \in \Xi_{\delta}$. We denote by $\mathbf{Q}_{\delta}\left(x_{\delta}, \varrho\right)$ the discrete domain consisting of the square of sidelength $\varrho$, centered at $x_{\delta}$, with horizontal and vertical sides. Let $\Lambda_{\delta}$ be the connected component of $\Xi_{\delta} \cap \mathbf{Q}\left(x_{\delta}, \varrho\right)$ containing $x_{\delta}$, and suppose $\varrho>0$ is small enough so that $s_{\delta} \notin \Lambda_{\delta}$. Denote by $l_{\delta}$ the arc of $\partial \Lambda_{\delta}$ that separates $x_{\delta}$ from $s_{\delta}$ in $\Xi_{\delta}$. We denote by $\mathbf{Q}_{\Xi_{\delta}}\left(x_{\delta}, \varrho, s_{\delta}\right)$ the connected component of $\Xi_{\delta} \backslash l_{\delta}$ containing $x_{\delta}$ (see Figure 13.7). We denote by $\Gamma\left(l_{\delta}\right)$ the set of corners of $l_{\delta}$, i.e. the points of $l_{\delta}$ where a horizontal and a vertical segment of $l_{\delta}$ intersect.

Proof of Theorem 29. First make the following observations: 


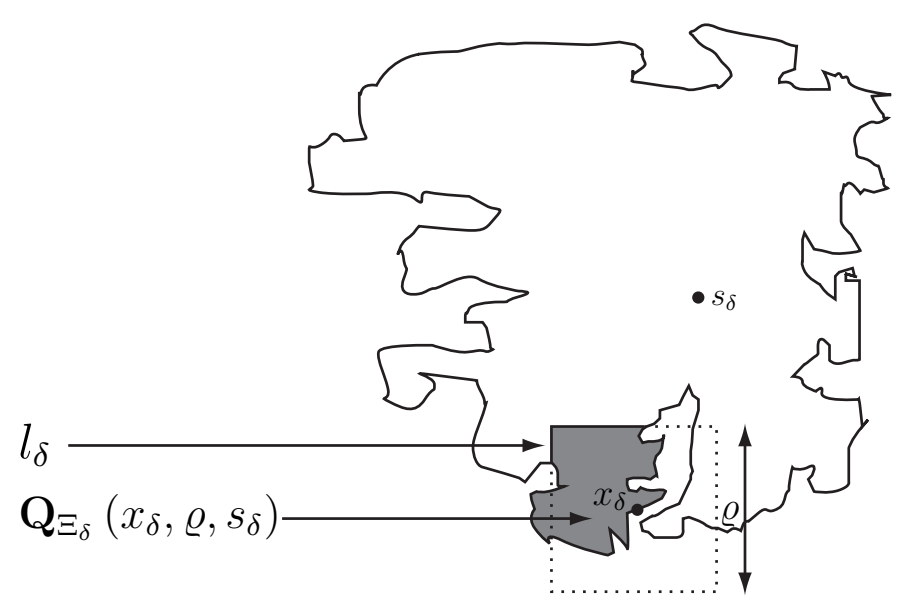

FiguRE 13.7. Localization near the point $x_{\delta}$

- By Lemmas 63 and 64 , we have

$$
\begin{aligned}
\mathbb{E}_{\Theta_{\delta}}^{\left[y_{\delta}, t_{\delta}\right]_{+}}\left[\sigma_{x_{\delta}}\right] & =\cos \left(\frac{\pi}{8}\right)\left|f_{\delta}^{\mathrm{FK}}\left(\Theta_{\delta}, y_{\delta}, t_{\delta}, x_{\delta}\right)\right| \\
\mathbb{E}_{\Xi_{\delta}}^{\text {free }}\left[\sigma_{x_{\delta}} \sigma_{s_{\delta}}\right] & =(\sqrt{2}+1)\left|f_{\delta}^{\mathrm{SPIN}}\left(\Xi_{\delta}, s_{\delta}, x_{\delta}\right)\right| .
\end{aligned}
$$

- By Lemma 73 , we have

$$
\begin{aligned}
\frac{\left\langle\sigma_{x} \sigma_{s}\right\rangle_{\Xi}^{\text {free }}}{\left\langle\sigma_{x}\right\rangle_{\Theta}^{[y, t]_{+}}} & =\frac{\sqrt{2}+1}{\cos \left(\frac{\pi}{8}\right)}\left|\frac{f^{\mathrm{SPIN}}(\Xi, s, x)}{f^{\mathrm{FK}}(\Theta, y, t, x)}\right|, \\
\frac{\left\langle\sigma_{x}\right\rangle_{\tilde{\Theta}}^{\left[\tilde{y}_{\delta}, \tilde{t}_{\delta}\right]_{+}}}{\left\langle\sigma_{x}\right\rangle_{\Theta}^{\left[y_{\delta}, t_{\delta}\right]_{+}}} & =\left|\frac{f^{\mathrm{FK}}(\tilde{\Theta}, \tilde{y}, \tilde{t}, x)}{f^{\mathrm{FK}}(\Theta, y, t, x)}\right| .
\end{aligned}
$$

- By Theorem 81 we have that

$$
\frac{1}{\delta}\left(f_{\delta}^{\mathrm{FK}}\right)^{2}\left(\Theta_{\delta}, y_{\delta}, t_{\delta}, \cdot\right) \underset{\delta \rightarrow 0}{\longrightarrow}\left(f^{\mathrm{FK}}\right)^{2}(\Theta, y, t, \cdot),
$$

on the compact subsets of $\Theta$. By changing if necessary the sign of $f_{\delta}^{\mathrm{FK}}$ (and choosing an arbitrary branch of the square root to define $f^{\mathrm{FK}}$ ) we can suppose that

$$
\frac{1}{\sqrt{\delta}} f_{\delta}^{\mathrm{FK}}\left(\Theta_{\delta}, y, t, \cdot\right) \underset{\delta \rightarrow 0}{\longrightarrow} f^{\mathrm{FK}}(\Theta, y, t, \cdot)
$$

- By Theorem 84, we have

$$
\frac{1}{\delta} f_{\delta}^{\mathrm{SPIN}}\left(\Xi_{\delta}, s_{\delta}, \cdot\right) \underset{\delta \rightarrow 0}{\longrightarrow} f^{\mathrm{SPIN}}(\Xi, s, \cdot)
$$

on the compact subsets of $\Xi$.

We now want to localize our observables in a neighborhood of $x_{\delta}$. 
- Take $\varrho>0$ small enough so that $\mathbf{Q}_{\Theta_{\delta}}\left(x_{\delta}, \varrho, y_{\delta}\right)=\mathbf{Q}_{\Xi_{\delta}}\left(x_{\delta}, \varrho, s_{\delta}\right)$ (see Definition 85 and Figure 13.7).

- Set $\Omega_{\delta}:=\mathbf{Q}_{\Theta_{\delta}}\left(x_{\delta}, \varrho, y_{\delta}\right)$.

- Let $\partial^{\mathrm{r}} \Omega_{\delta} \subset \partial \Omega_{\delta}$ (the "rough part of the boundary") be such that

$$
\partial^{\mathrm{r}} \Omega_{\delta}=\partial \Omega_{\delta} \cap \partial \Theta_{\delta}=\partial \Omega_{\delta} \cap \partial \Xi_{\delta},
$$

In other words, the arc $\partial^{\mathrm{r}} \Omega_{\delta}$ is the arc of the boundary of $\Omega_{\delta}$ that is common to $\Theta_{\delta}$ and $\Xi_{\delta}$.

- Let $\partial^{\mathrm{s}} \Omega_{\delta}$ be $\partial \Omega_{\delta} \backslash \partial^{\mathrm{r}} \Omega_{\delta}$ : it is the arc which is made of the sides of the square $\mathbf{Q}_{\delta}\left(x_{\delta}, \varrho\right)$ (it is equal to $l_{\delta}$ in Definition 85).

- Let $D>0$ be such that $\Omega_{\delta} \subset \mathbf{D}(0, D)$ for all $\delta>0$.

- Let $d>0$ be such that $\operatorname{dist}\left(x_{\delta}, \partial^{\mathrm{s}} \Omega_{\delta}\right) \geq d$ for all $\delta>0$ and such that we can choose a point $w_{\delta}$ away from the corners and the "rough part of the boundary", with dist $\left(w_{\delta}, \partial^{\mathrm{r}} \Omega_{\delta} \cup \Gamma\left(l_{\delta}\right)\right) \geq d$ for all $\delta>0$.

Define $u_{\delta}^{\mathrm{FK}}: \partial_{0} \mathcal{V}_{\Omega_{\delta}^{m}} \rightarrow \mathbb{C}$ by

$$
u_{\delta}^{\mathrm{FK}}(z):= \begin{cases}\mathrm{P}_{\nu_{\text {in }}^{-\frac{1}{2}}(z)}\left[\frac{1}{\sqrt{\delta}} f_{\delta}^{\mathrm{FK}}\left(\Theta_{\delta}, y, t, z\right)\right] & z \in \partial_{0}^{\mathrm{s}} \mathcal{V}_{\Omega_{\delta}^{m}} \\ 0 & z \in \partial_{0}^{\mathrm{r}} \mathcal{V}_{\Omega_{\delta}^{m}}\end{cases}
$$

and $u^{\mathrm{FK}}(\cdot): \partial \Omega \rightarrow \mathbb{C}$ by

$$
u^{\mathrm{FK}}(z):= \begin{cases}\mathrm{P}_{\nu_{\mathrm{in}}^{-\frac{1}{2}}(z)}\left[f^{\mathrm{FK}}\left(\Theta_{\delta}, y, t, z\right)\right] & z \in \partial_{0}^{\mathrm{s}} \mathcal{V}_{\Omega_{\delta}^{m}}, \\ 0 & z \in \partial_{0}^{\mathrm{r}} \mathcal{V}_{\Omega_{\delta}^{m}}\end{cases}
$$

Assume without loss of generality that $\nu_{\text {in }}\left(w_{\delta}\right)=1$, so that $f_{\delta}^{\text {SPIN }}\left(\Omega_{\delta}, w_{\delta}, \cdot\right)=$ $g_{\delta}^{\mathrm{SPIN}}\left(\Omega_{\delta}, w_{\delta}, \cdot\right)$ and $f^{\mathrm{SPIN}}\left(\Omega_{\delta}, w_{\delta}, \cdot\right)=g^{\mathrm{SPIN}}\left(\Omega_{\delta}, w_{\delta}, \cdot\right)$.

Let $\epsilon>0$. We want to show that there exists a $\delta_{0}$ (depending only on $\varrho, d, D$ ) such that for any $\delta \leq \delta_{0}$,

$$
\left|\frac{\frac{1}{\sqrt{\delta}} f_{\delta}^{\mathrm{FK}}\left(\Theta_{\delta}, y, t, x_{\delta}\right)}{\frac{1}{\delta} f_{\delta}^{\mathrm{SPIN}}\left(\Omega_{\delta}, w_{\delta}, x_{\delta}\right)}-\frac{f^{\mathrm{FK}}\left(\Theta_{\delta}, y, t, x_{\delta}\right)}{f^{\mathrm{SPIN}}\left(\Omega_{\delta}, w_{\delta}, x_{\delta}\right)}\right| \leq \epsilon .
$$

Let us first use the convolution representations introduced in Sections 13.4.1 and 13.7.1.

- By Lemma 67, we have that

$$
\begin{aligned}
& \frac{\frac{1}{\sqrt{\delta}} f_{\delta}^{\mathrm{FK}}\left(\Theta_{\delta}, y, t, x_{\delta}\right)}{\frac{1}{\delta} f_{\delta}^{\mathrm{SPIN}}\left(\Omega_{\delta}, w_{\delta}, x_{\delta}\right)}=\frac{\mathbb{K}_{\delta}\left[\Omega_{\delta},\left.u_{\delta}^{\mathrm{FK}}\right|_{\partial_{0}^{\mathrm{s}}}{\mathcal{\Omega _ { \delta }}}_{\delta}\right]\left(x_{\delta}\right)}{\frac{1}{\delta} g_{\delta}^{\mathrm{SPIN}}\left(\Omega_{\delta}, w_{\delta}, x_{\delta}\right)} \\
& =\sum_{z_{\delta} \in \partial_{0}^{\mathrm{s}} \mathcal{V}_{\Omega_{\delta}^{m}}} u_{\delta}^{\mathrm{FK}}\left(z_{\delta}\right) \frac{g_{\delta}^{\mathrm{SPIN}}\left(\Omega_{\delta}, z_{\delta}, x_{\delta}\right)}{g_{\delta}^{\mathrm{SPIN}}\left(\Omega_{\delta}, w_{\delta}, x_{\delta}\right)} \delta \\
& =\sum_{z_{\delta} \in \partial_{0}^{\mathrm{s}} \mathcal{V}_{\Omega_{\delta}^{m}}} u_{\delta}^{\mathrm{FK}}\left(z_{\delta}\right) \frac{\overline{g_{\delta}^{\mathrm{SPIN}}\left(\Omega_{\delta}, x_{\delta}, z_{\delta}\right)}}{\overline{g_{\delta}^{\mathrm{SPIN}}\left(\Omega_{\delta}, x_{\delta}, w_{\delta}\right)}} \delta .
\end{aligned}
$$


- Similarly, by Proposition 79, we have

$$
\begin{aligned}
\frac{f^{\mathrm{FK}}\left(\Theta_{\delta}, y, t, x_{\delta}\right)}{f^{\mathrm{SPIN}}\left(\Omega_{\delta}, w_{\delta}, x_{\delta}\right)} & =\frac{\mathbb{K}\left[\Omega,\left.u^{\mathrm{FK}}\right|_{\partial^{\mathrm{s}} \Omega_{\delta}}\right]\left(x_{\delta}\right)}{g^{\mathrm{SPIN}}\left(\Omega_{\delta}, w_{\delta}, x_{\delta}\right)} \\
& =\int_{\partial_{0}^{\mathrm{s}} \Omega_{\delta}} u^{\mathrm{FK}}(z) \frac{g^{\mathrm{SPIN}}\left(\Omega_{\delta}, z, x_{\delta}\right)}{g^{\mathrm{SPIN}}\left(\Omega_{\delta}, w_{\delta}, x_{\delta}\right)} \mathrm{d}|z| \\
& =\int_{\partial_{0}^{\mathrm{s}} \Omega_{\delta}} u^{\mathrm{FK}}(z) \frac{\frac{g^{\mathrm{SPIN}}\left(\Omega_{\delta}, x_{\delta}, z\right)}{g^{\mathrm{SPIN}}\left(\Omega_{\delta}, x_{\delta}, w_{\delta}\right)}}{\mathrm{S}}|z|,
\end{aligned}
$$

where $u^{\mathrm{FK}}: \partial_{0}^{\mathrm{s}} \Omega_{\delta} \rightarrow \mathbb{C}$ is defined by

$$
u^{\mathrm{FK}}(z):=\mathrm{P}_{\nu_{\text {in }}(z)}\left[f^{\mathrm{FK}}\left(\Theta_{\delta}, y, t, z\right)\right] .
$$

In order to prove the theorem, we prove the convergence of the discrete convolution representation above to the continuous one. The integrand in the convolution converges away from the "rough part" and the corners (Theorems 81 and 83), so we just need to control the values of this integrand near the corners and the "rough part". For this, we use a priori estimates which will be proven in the next subsection.

Now, set $\psi_{\delta}(\cdot):=g_{\delta}^{\mathrm{SPIN}}\left(\Omega_{\delta}, x_{\delta}, \cdot\right)$ and $\psi(\cdot):=g^{\mathrm{SPIN}}\left(\Omega_{\delta}, x_{\delta}, \cdot\right)$. All the estimates below will be depend on $\varrho, d, D$ only.

- By Proposition 86 in the next subsection, there exists $C^{\mathrm{FK}}$ and $\vartheta>0$ such that

$$
\left|u_{\delta}^{\mathrm{FK}}\left(z_{\delta}\right)\right| \leq \frac{C^{\mathrm{FK}}}{\operatorname{dist}\left(z_{\delta},\left[t_{\delta}, y_{\delta}\right]\right)^{\frac{1}{2}-\vartheta}} .
$$

- By Lemma 87 in the next subsection, there exists $C>0$ such that

$$
\left|\frac{\psi_{\delta}\left(z_{\delta}\right)}{\psi_{\delta}\left(w_{\delta}\right)}\right| \leq \frac{C}{\sqrt{\operatorname{dist}\left(z_{\delta}, \partial^{\mathrm{r}} \Omega_{\delta} \cup \Gamma\left(l_{\delta}\right)\right)}} .
$$

Hence, for any $\epsilon>0$, we can find $\theta>0$ and split $\partial^{\mathrm{s}} \Omega_{\delta}$ into $\partial^{\mathrm{b}} \Omega_{\delta} \cup \partial^{\mathrm{i}} \Omega_{\delta}$ in such a way that for any $\delta>0$ :

(1) $\operatorname{dist}\left(\partial^{\mathrm{i}} \Omega_{\delta}, \partial^{\mathrm{r}} \Omega_{\delta} \cup \Gamma\left(\Omega_{\delta}\right)\right) \geq \theta$

(2) $\sum_{z_{\delta} \in \partial_{0}^{\mathfrak{b}} \mathcal{\Omega}_{\Omega_{\delta}^{m}}}\left|u_{\delta}^{\mathrm{FK}}\left(z_{\delta}\right)\right|\left|\frac{\psi_{\delta}\left(z_{\delta}\right)}{\psi\left(w_{\delta}\right)}\right| \cdot \delta \leq \frac{\epsilon}{6}$

(3) $\int_{\partial_{0}^{\mathrm{b}} \Omega_{\delta}}\left|u^{\mathrm{FK}}(z)\right|\left|\frac{\psi(z)}{\psi\left(w_{\delta}\right)}\right| \mathrm{d}|z| \leq \frac{\epsilon}{6}$.

We obtain

$$
\begin{aligned}
& \left|\frac{\frac{1}{\sqrt{\delta}} f_{\delta}^{\mathrm{FK}}\left(\Theta_{\delta}, y, t, x_{\delta}\right)}{\frac{1}{\delta} f_{\delta}^{\mathrm{SPIN}}\left(\Omega_{\delta}, w_{\delta}, x_{\delta}\right)}-\frac{f^{\mathrm{FK}}\left(\Theta_{\delta}, y, t, x_{\delta}\right)}{f^{\mathrm{SPIN}}\left(\Omega_{\delta}, w_{\delta}, x_{\delta}\right)}\right| \\
= & \left|\sum_{z_{\delta} \in \partial_{0}^{\mathrm{s}} \mathcal{V}_{\Omega_{\delta}^{m}}} u_{\delta}^{\mathrm{FK}}\left(z_{\delta}\right) \frac{\psi_{\delta}\left(z_{\delta}\right)}{\psi_{\delta}\left(w_{\delta}\right)} \cdot \delta-\int_{\partial^{\mathrm{s}} \Omega_{\delta}} u^{\mathrm{FK}}(z) \frac{\psi(z)}{\psi\left(w_{\delta}\right)} \mathrm{d}\right| z|| \\
\leq & \frac{\epsilon}{3}+\mathbf{A}_{\delta}
\end{aligned}
$$


where

$$
\mathbf{A}_{\delta}:=\left|\sum_{z \delta} u_{0}^{\mathrm{i} \nu_{\Omega_{\delta}^{m}}} u_{\delta}^{\mathrm{FK}}\left(z_{\delta}\right) \frac{\psi_{\delta}\left(z_{\delta}\right)}{\psi_{\delta}\left(w_{\delta}\right)} \cdot \delta-\int_{\partial^{\mathrm{i}} \Omega_{\delta}} u^{\mathrm{FK}}(z) \frac{\psi(z)}{\psi\left(w_{\delta}\right)} \mathrm{d}\right| z|| .
$$

We can write

$$
\mathbf{A}_{\delta} \leq \mathbf{B}_{\delta}+\mathbf{C}_{\delta}
$$

where

$$
\begin{aligned}
& \mathbf{B}_{\delta}:=\left|\sum_{z_{\delta} \in \partial_{0}^{\mathrm{i}} \mathcal{V}_{\Omega_{\delta}^{m}}} u_{\delta}^{\mathrm{FK}}\left(z_{\delta}\right) \frac{\psi_{\delta}\left(z_{\delta}\right)}{\psi_{\delta}\left(w_{\delta}\right)} \cdot \delta-\sum_{z_{\delta} \in \partial_{0}^{\mathrm{i}} \mathcal{V}_{\Omega_{\delta}^{m}}} u^{\mathrm{FK}}\left(z_{\delta}\right) \frac{\psi\left(z_{\delta}\right)}{\psi\left(w_{\delta}\right)} \cdot \delta\right|, \\
& \mathbf{C}_{\delta}:=\left|\sum_{z_{\delta} \in \partial_{0}^{\mathrm{i}} \mathcal{V}_{\Omega_{\delta}^{m}}} u^{\mathrm{FK}}\left(z_{\delta}\right) \frac{\psi\left(z_{\delta}\right)}{\psi\left(w_{\delta}\right)} \cdot \delta-\int_{\partial^{\mathrm{i}} \Omega_{\delta}} u^{\mathrm{FK}}(z) \frac{\psi(z)}{\psi\left(w_{\delta}\right)} \mathrm{d}\right| z|| .
\end{aligned}
$$

Let us estimate $\mathbf{B}_{\delta}$ first. From the following two observations, we get that there exists $\delta_{\mathbf{B}}>0$ such that for any $\delta \leq \delta_{\mathbf{B}}, \mathbf{B}_{\delta} \leq \epsilon / 3$.

- By Theorem 81, for any $\epsilon_{1}>0$, we have that there exists $\delta_{1}>0$ such that for $\delta \leq \delta_{1},\left|u_{\delta}^{\mathrm{FK}}(z)-u^{\mathrm{FK}}(z)\right| \leq \delta_{1}$ on $\partial_{0}^{\mathrm{i}} \mathcal{V}_{\Omega_{\delta}^{m}}$.

- By Theorem 83 and Theorem 83 , for any $\epsilon_{2}>0$, there exists $\delta_{2}>0$ such that for $\delta \leq \delta_{2}$,

$$
\left|\frac{\psi_{\delta}\left(z_{\delta}\right)}{\psi_{\delta}\left(w_{\delta}\right)}-\frac{\psi\left(z_{\delta}\right)}{\psi\left(w_{\delta}\right)}\right| \leq \epsilon_{2} \quad \forall z_{\delta} \in \partial_{0}^{\mathrm{i}} \mathcal{V}_{\Omega_{\delta}^{m}} .
$$

From standard calculus, there exists $\delta_{\mathbf{C}}>0$ such that for any $\delta \leq \delta_{\mathbf{C}}, \mathbf{C}_{\delta} \leq \epsilon / 3$. Hence taking $\delta_{0}=\min \left(\delta_{\mathbf{B}}, \delta_{\mathbf{C}}\right)$, we obtain the desired inequality (Eq. 13.10). We therefore obtain

$$
\frac{\frac{1}{\sqrt{\delta}} f_{\delta}^{\mathrm{FK}}\left(\Theta_{\delta}, y_{\delta}, t_{\delta}, x\right)}{\frac{1}{\delta} f_{\delta}^{\mathrm{SPIN}}\left(\Omega_{\delta}, w_{\delta}, x_{\delta}\right)} \rightarrow \frac{f^{\mathrm{FK}}(\Theta, y, t, x)}{f^{\mathrm{SPIN}}(\Omega, w, x)},
$$

noticing that the right-hand side is Carathéodory-stable (see Lemma 80).

Repeating the arguments above, replacing $\frac{1}{\sqrt{\delta}} f_{\delta}^{\mathrm{FK}}\left(\Theta_{\delta}, y_{\delta}, t_{\delta}, x_{\delta}\right)$ by $\frac{1}{\delta} f_{\delta}^{\mathrm{SPIN}}\left(\Xi_{\delta}, s_{\delta}, x_{\delta}\right)$, we obtain

$$
\frac{\frac{1}{\delta} f_{\delta}^{\text {SPIN }}\left(\Xi_{\delta}, s_{\delta}, x_{\delta}\right)}{\frac{1}{\delta} f_{\delta}^{\text {SPIN }}\left(\Omega_{\delta}, w_{\delta}, x_{\delta}\right)} \rightarrow \frac{f^{\text {SPIN }}(\Xi, s, x)}{f^{\text {SPIN }}(\Omega, w, x)} .
$$

Taking the ratio of the convergence results 13.11 and 13.12 and noticing that the limits are both nonzero (Lemma 78), we obtain the first convergence result of the theorem. The second one is obtained by using exactly the same method.

13.10. A priori estimates for holomorphic observables. In this subsection, we derive the technical lemmas that have been used in Section 13.9. Most importantly, these estimates are uniform with respect to the mesh size $\delta>0$. 
Lemma 86. There exists a universal $\vartheta>0$ such that for any $d, D, \varrho>0$, there exists $C^{\mathrm{FK}}(d, D, r)$ such that for any discrete domain $\left(\Omega_{\delta}, r_{\delta}, \ell_{\delta}\right)$, we have

$$
\left|\frac{1}{\sqrt{\delta}} f_{\delta}^{\mathrm{FK}}\left(\Omega_{\delta}, r_{\delta}, \ell_{\delta}, z_{\delta}\right)\right| \leq \frac{C^{\mathrm{FK}}}{\left(\operatorname{dist}\left(z_{\delta}, \partial \Omega_{\delta}\right)\right)^{\frac{1}{2}-\vartheta}}
$$

for any $z_{\delta} \in \mathcal{V}_{\Omega_{\delta}^{m}}$ with dist $\left(z_{\delta},\left\{r_{\delta}, \ell_{\delta}\right\}\right) \geq d$. Similarly there exists $C^{\text {SPIN }}(d, D, r)$ such that for any discrete domain $\left(\Omega_{\delta}, p_{\delta}^{1}, a_{\delta}, p_{\delta}^{2}\right)$ with dist $\left(p_{\delta}^{1}, p_{\delta}^{2}\right) \geq d$, dist $\left(a_{\delta},\left[p_{\delta}^{2}, p_{\delta}^{1}\right]\right) \geq$ $d$ and with $\operatorname{diam}\left(\Omega_{\delta}\right) \leq D$, and for any $z_{\delta} \in \mathcal{V}_{\Omega_{\delta}^{m}}$ with $\operatorname{dist}\left(z_{\delta}, a_{\delta}\right) \geq \varrho$, we have

$$
\left|\frac{1}{\delta} f_{\delta}^{\mathrm{SPIN}}\left(\Omega_{\delta}, a_{\delta}, z_{\delta}\right)\right| \leq \frac{C^{\mathrm{SPIN}}}{\left(\operatorname{dist}\left(z_{\delta}, \partial \Omega_{\delta}\right)\right)^{\frac{1}{2}-\vartheta}} .
$$

The proof is given in Section 13.10.4.

Lemma 87. Let $r, R>0$. Let $\left(\Omega_{\delta}, p_{\delta}^{1}, p_{\delta}^{2}, x_{\delta}\right)$ be a discrete domain with $\operatorname{diam}\left(\Omega_{\delta}\right) \leq$ $R$, with $\left[p_{\delta}^{1}, p_{\delta}^{2}\right]$ being a straight part of $\partial \Omega_{\delta}$, with $\operatorname{dist}\left(p_{\delta}^{1}, p_{\delta}^{2}\right) \geq r, w_{\delta} \in\left[p_{\delta}^{1}, p_{\delta}^{2}\right]$, $\operatorname{dist}\left(w_{\delta},\left[p_{\delta}^{2}, p_{\delta}^{1}\right]\right) \geq r$, dist $\left(x_{\delta},\left[p_{\delta}^{1}, p_{\delta}^{2}\right]\right) \geq r$. Then there exists $C$, dependent on $r, R$ only.

for all $z_{\delta}$ on $\left[p_{\delta}^{1}, p_{\delta}^{2}\right]$.

$$
\left|\frac{g_{\delta}^{\mathrm{SPIN}}\left(\Omega_{\delta}, x_{\delta}, z_{\delta}\right)}{g_{\delta}^{\mathrm{SPIN}}\left(\Omega_{\delta}, x_{\delta}, w_{\delta}\right)}\right| \leq \frac{C}{\sqrt{\operatorname{dist}\left(z_{\delta},\left\{p_{\delta}^{1}, p_{\delta}^{2}\right\}\right)}}
$$

The proof is given in Section 13.10.4.

13.10.1. Discrete complex analysis techniques.

Definition 88. For a function $f: \overline{\mathcal{V}}_{\Omega_{\delta}^{*}} \rightarrow \mathbb{C}$, we define the Laplacian by $\Delta_{\circ} f:$ $\mathcal{V}_{\Omega_{\delta}^{*}} \rightarrow \mathbb{C}$ by

$$
\Delta_{\circ} f(v):=\sum_{w \in \overline{\mathcal{V}}_{\Omega_{\delta}^{*}: v \sim w}} f(w)-f(v) .
$$

For a function $f: \overline{\mathcal{V}}_{\Omega_{\delta}} \rightarrow \mathbb{C}$ and an arc $\mathfrak{a} \subset \partial \mathcal{V}_{\Omega_{\delta}}$ following [ChSm09] (see also [Hon10a, Section 2.6.1] for a setup closer to the one employed here), we define the (modified) Laplacian $\tilde{\Delta}_{\bullet} f: \mathcal{V}_{\Omega_{\delta}} \rightarrow \mathbb{C}$ by

$$
\tilde{\Delta}_{\bullet} f_{\delta}(v):=2 \alpha\left(\sum_{w \in \partial \mathcal{V}_{\delta}: w \sim v} f(w)-f(v)\right)+\sum_{w \in \mathcal{V}_{\Omega_{\delta}}: w \sim v} f(w)-f(v),
$$

where $\alpha=\sqrt{2}-1$ as usual.

13.10.2. Discrete integral of the square. Except in specific cases, the product (or even the square) of s-holomorphic functions is no longer discrete holomorphic. However, a remarkable feature of s-holomorphic functions is that the (real part of the) antiderivative of the square of an s-holomorphic function can be still defined. It turns out to be particularly useful to integrate the square of the observables $g_{\delta}^{\mathrm{FK}}$ and $g_{\delta}^{\text {SPIN }}$, as the boundary of values of the resulting functions are much simpler.

The following lemma was introduced in [Smi10a, Lemma 3.6]. An important simplification of the boundary value was introduced in [ChSm09, Section 3.6].

Proposition 89. Let $\left(\Omega_{\delta}, r, \ell\right)$ be a discrete domain and set $f_{\delta}(\cdot):=\frac{1}{\sqrt{\delta}} f_{\delta}^{\mathrm{FK}}\left(\Omega_{\delta}, r, \ell, \cdot\right)$. There exists a unique function $\mathbb{I} f_{\delta}: \mathcal{V}_{\Omega_{\delta}} \cup \mathcal{V}_{\Omega_{\delta}^{*}} \cup \partial \mathcal{V}_{[\ell, r]^{*}} \rightarrow \mathbb{R}$ such that 
- $\mathbb{I} f_{\delta}$ is equal to 0 on $\mathcal{V}_{[\ell, r]^{*}}$.

- $\mathbb{I} f_{\delta}$ is equal to 1 on $\mathcal{V}_{[r, \ell]}$.

- If $e=\langle x, y\rangle \in \mathcal{E}_{\Omega_{\delta}^{m}}$ is a edge and $b \in \mathcal{V}_{\Omega_{\delta}}$ and $w \in \mathcal{V}_{\Omega_{\delta}^{*}} \cup \partial \mathcal{V}_{[\ell, r]^{*}}$ are such that $\langle b, w\rangle=e^{*}$, then

$$
\mathbb{I} f_{\delta}(b)-\mathbb{I} f_{\delta}(w)=\sqrt{2} \delta\left|\mathrm{P}_{\ell(e)}\left[f_{\delta}(x)\right]\right|^{2}=\sqrt{2} \delta\left|\mathrm{P}_{\ell(e)}\left[f_{\delta}(y)\right]\right|^{2} .
$$

- For any $v, w \in \mathcal{V}_{\Omega_{\delta}}$ with $v \sim w$ or any $v, w \in \mathcal{V}_{\Omega_{\delta}^{*}} \cup \partial \mathcal{V}_{[\ell, r]^{*}}$ with $v \sim w$, we have $\mathbb{I} f_{\delta}(v)-\mathbb{I} f_{\delta}(w)=-\Re \mathfrak{e}\left(f_{\delta}^{2}(m)(v-w)\right)$, where $m \in \mathcal{V}_{\Omega_{\delta}^{m}}$ is the midpoint of $\langle v, w\rangle$.

- The function $\mathbb{I} f_{\delta}$ can be extended to $\partial \mathcal{V}_{[\ell, r]}$ by setting its value to 0 there, in such a way that

$$
\begin{aligned}
& -\Delta_{\circ} \mathbb{I} f_{\delta}(z) \leq 0, \text { for all } z \in \mathcal{V}_{\Omega_{\delta}^{*}} \backslash \mathcal{V}_{[r, \ell]^{*}} . \\
& -\tilde{\Delta} . \mathbb{I} f_{\delta}(z) \geq 0, \text { for all } z \in \mathcal{V}_{\Omega_{\delta}} \backslash \mathcal{V}_{[r, \ell]}
\end{aligned}
$$

Proof. This follows from [ChSm09, Remark 3.15]. The boundary modification trick that we use is of the same form as in [Hon10a, Section 2.6.1].

Remark 90 . The function $\mathbb{I} f$ can also be extended to $\partial \mathcal{V}_{[r, \ell]^{*}}$ by the value 1 but we will not need this here.

Similarly, we can construct the antiderivative of the square of the spin observable.

Proposition 91. Let $\left(\Omega_{\delta}, x\right)$ be a discrete domain and set $f_{\delta}(\cdot):=\frac{1}{\delta} f_{\delta}^{\text {SPIN }}\left(\Omega_{\delta}, x, \cdot\right)$. Denote by $x_{\text {in }} \in \mathcal{V}_{\Omega_{\delta}}$ and $x_{\text {out }} \in \partial \mathcal{V}_{\Omega_{\delta}}$ the endpoints of the edge of $\partial \mathcal{E}_{\Omega_{\delta}}$ whose midpoint is $x$. There exists a unique function $\mathbb{I} f_{\delta}: \mathcal{V}_{\Omega_{\delta}} \cup \mathcal{V}_{\Omega_{\delta}^{*}} \cup \partial \mathcal{V}_{\Omega_{\delta}^{*}} \rightarrow \mathbb{R}$ such that

- $I f_{\delta}$ is equal to 0 on $\partial \mathcal{V}_{\Omega_{\delta}^{*}}$

- If $e=\langle x, y\rangle \in \mathcal{E}_{\Omega_{\delta}^{m}}$ is a edge and $b \in \mathcal{V}_{\Omega_{\delta}}$ and $w \in \mathcal{V}_{\Omega_{\delta}^{*}} \cup \partial \mathcal{V}_{\Omega_{\delta}^{*}}$ are such that $\langle b, w\rangle=e^{*}$, then

$$
\mathbb{I} f_{\delta}(b)-\mathbb{I} f_{\delta}(w)=\sqrt{2} \delta\left|\mathrm{P}_{\ell(e)}\left[f_{\delta}(x)\right]\right|^{2}=\sqrt{2} \delta\left|\mathrm{P}_{\ell(e)}\left[f_{\delta}(y)\right]\right|^{2} .
$$

- For any $v, w \in \mathcal{V}_{\Omega_{\delta}}$ with $v \sim w$ or any $v, w \in \mathcal{V}_{\Omega_{\delta}^{*}} \cup \partial \mathcal{V}_{\Omega_{\delta}^{*}}$ with $v \sim w$, we have $\mathbb{I} f_{\delta}(v)-\mathbb{I} f_{\delta}(w)=-\Re \mathfrak{e}\left(f_{\delta}^{2}(m)(v-w)\right)$, where $m \in \mathcal{V}_{\Omega_{\delta}^{m}}$ is the midpoint of $\langle v, w\rangle$.

- The function $\mathbb{I} f_{\delta}$ can be extended to $\partial \mathcal{V}_{\Omega_{\delta}} \backslash\left\{x_{\text {out }}\right\}$, by the value 0 , in such a way that

$$
\begin{aligned}
& -\Delta_{\circ} \mathbb{I} f_{\delta}(z) \leq 0 \text { for all } z \in \mathcal{V}_{\Omega_{\delta}^{*}}, \\
& -\tilde{\Delta} \mathbb{I} f_{\delta}(z) \geq 0 \text { for all } z \in \mathcal{V}_{\Omega_{\delta}} \backslash\left\{x_{\mathrm{in}}\right\} .
\end{aligned}
$$

Proof. See [ChSm09, Remark 3.15] or [Hon10a, Section 2.6.1].

Definition 92. Given a discrete domain $\Omega_{\delta}$ and an s-holomorphic function $h_{\delta}$ : $\mathcal{V}_{\Omega_{\delta}^{m}} \rightarrow \mathbb{C}$, we define the discrete antiderivative of $g^{2}$ as the function on $\mathcal{V}_{\Omega_{\delta}} \cup \mathcal{V}_{\Omega_{\delta}^{*}} \cup$ $\partial \mathcal{V}_{\Omega_{\delta}^{*}}$ obtained by integrating Equation 13.14 and denote it by $\mathbb{I} h_{\delta}$

Remark 93. It is always possible to integrate the Equation 13.14 if $g$ is s-holomorphic, and this defines $\mathbb{I} g$ uniquely, up to an additive constant.

13.10.3. Control of s-holomorphic functions. Let us give a very useful lemma, introduced in [ChSm09], that allows to control the s-holomorphic functions given the integral of their square. 
Proposition 94. Let $f_{\delta}: \mathcal{V}_{\Omega_{\delta}^{m}} \rightarrow \mathbb{C}$ be an s-holomorphic function and let $\mathbb{I} f_{\delta}$ be a discrete antiderivative of $f_{\delta}^{2}$. Then there exists a constant $C>0$ such that for $\delta>0$ and any $x \in \mathcal{V}_{\Omega_{\delta}^{m *}}$, we have

$$
\begin{aligned}
\left|f_{\delta}(v)\right|^{2} & \leq C \cdot \frac{\max _{w \in \mathcal{V}_{\Omega_{\delta}^{m *}}}\left|\mathbb{I}\left[f_{\delta}\right](w)\right|}{\operatorname{dist}\left(v, \partial_{0} \mathcal{V}_{\Omega_{\delta}^{m}}\right)} \forall v \in \mathcal{V}_{\Omega_{\delta}^{m}} . \\
\frac{1}{\delta}\left\|\nabla_{\delta} f_{\delta}(x)\right\|^{2} & \leq C \cdot \frac{\max _{w \in \mathcal{V}_{\Omega_{\delta}^{m *}}}\left|\mathbb{I}\left[f_{\delta}\right](w)\right|}{\operatorname{dist}\left(x, \partial_{0} \mathcal{V}_{\Omega_{\delta}^{m}}\right)^{3}} \forall x \in \mathcal{V}_{\Omega_{\delta}} \cup \mathcal{V}_{\Omega_{\delta}^{*}},
\end{aligned}
$$

where

$$
\nabla_{\delta} f_{\delta}(x):=\left(f_{\delta}\left(x+\frac{\delta}{2}\right)-f_{\delta}\left(x-\frac{\delta}{2}\right), f_{\delta}\left(x+i \frac{\delta}{2}\right)-f_{\delta}\left(x-\frac{i \delta}{2}\right)\right) .
$$

Proof. See [ChSm09, Theorem 3.12] or [?, Proposition 27].

13.10.4. Proofs of the lemmas. We can now give the proof of the lemmas given at the beginning of this subsection.

Proof of Lemma 86. Set $F_{\delta}^{\mathrm{FK}}:=\mathbb{I}\left[\frac{f_{\delta}^{\mathrm{FK}}\left(\Omega_{\delta}, r_{\delta}, \ell_{\delta}, \cdot\right)}{\sqrt{\delta}}\right]$ and $F_{\delta}^{\mathrm{SPIN}}:=\mathbb{I}\left[\frac{f_{\delta}^{\mathrm{SPIN}}\left(\Omega_{\delta}, a_{\delta}, \cdot\right)}{\delta}\right]$ (as in Propositions 89 and 91 .

By Proposition 89 and maximum principle, we have that $F_{\delta}^{\mathrm{FK}}$ is uniformly bounded by 1 . By the discrete Beurling estimate [ChSm11, Proposition 2.10], we have

$$
\left|F_{\delta}^{\mathrm{FK}}\left(z_{\delta}\right)\right| \leq C \cdot \operatorname{dist}\left(z_{\delta},\left[\ell_{\delta}, r_{\delta}\right]\right)^{2 \vartheta}
$$

for any $z_{\delta} \in \mathcal{V}_{\Omega_{\delta}^{m}}$ with $\operatorname{dist}\left(z_{\delta},\left\{r_{\delta}, \ell_{\delta}\right\}\right) \geq r$. Proposition 94 allows to deduce the first estimate.

Let us first show that $F_{\delta}^{\text {SPIN }}$ is uniformly bounded for $\left(\Omega_{\delta}, p_{\delta}^{1}, a_{\delta}, p_{\delta}^{2}\right)$ and $z_{\delta}$ satisfying the conditions above. By Theorem 84 we have $\frac{1}{\delta} f_{\delta}^{\text {SPIN }}$ is uniformly bounded on a contour separating $a_{\delta}$ from $z_{\delta}$, and by Proposition $91 F_{\delta}^{\text {SPIN }}$ is constant on $\partial \mathcal{V}_{\Omega_{\delta}^{*}} \cup\left(\partial \mathcal{V}_{\Omega_{\delta}} \backslash\{a\}\right)$. By subharmonicity and superharmonicity of the respective restrictions of $F_{\delta}^{\text {SPIN }}$ to $\mathcal{V}_{\Omega_{\delta}^{*}}$ and $\mathcal{V}_{\Omega_{\delta}} \backslash\{a\}$, we obtain the uniform boundedness of $F_{\delta}^{\text {SPIN }}$. Hence, again by Beurling estimate, $F_{\delta}^{\text {SPIN }}\left(z_{\delta}\right)=O\left(\operatorname{dist}\left(z_{\delta}, \partial \Omega_{\delta}\right)^{2 \vartheta}\right)$ as $z \rightarrow \partial \Omega$. By using Proposition 94, we obtain the second estimate.

Proof of Lemma 87. Note that

$$
\left|\frac{g_{\delta}^{\mathrm{SPIN}}\left(\Omega_{\delta}, x_{\delta}, \cdot\right)}{g_{\delta}^{\mathrm{SPIN}}\left(\Omega_{\delta}, x_{\delta}, w_{\delta}\right)}\right|=\left|\frac{f_{\delta}^{\mathrm{SPIN}}\left(\Omega_{\delta}, x_{\delta}, \cdot\right)}{\left|f_{\delta}^{\mathrm{SPIN}}\left(\Omega_{\delta}, x_{\delta}, w_{\delta}\right)\right|}\right|,
$$

so we will instead estimate the right-hand side. Suppose without loss of generality that $\left[p_{\delta}^{1}, p_{\delta}^{2}\right]$ is a vertical part and that $\Omega_{\delta}$ lies on the right of $\left[p_{\delta}^{1}, p_{\delta}^{2}\right]$. Consider the antiderivative $F_{\delta}$ of the square of $\frac{f_{\delta}^{\operatorname{SPIN}}\left(\Omega_{\delta}, x_{\delta}, \cdot\right)}{\left|f_{\delta}^{\operatorname{SPIN}}\left(\Omega_{\delta}, x_{\delta}, w_{\delta}\right)\right|}$ with 0 boundary value, as defined by Proposition 91. By [ChSm09, proof of Theorem 5.9], we have that at distance $r / 2$ from $x_{\delta} F_{\delta}$ is bounded by a constant $M=M(r, R)$. Let $S_{\delta}$ be a square of sidelength dist $\left(z_{\delta},\left\{p_{\delta}^{1}, p_{\delta}^{2}\right\}\right)$ such that $z_{\delta}$ lies at the middle of the left side of $S_{\delta}$. Let $H_{\delta}$ be a harmonic function with respect to a modified Laplacian (as discussed in ChSm09, Hon10a, DHN11) on $\overline{\mathcal{V}}_{S_{\delta}}$, with boundary value 0 on the left side of $S_{\delta}$ and boundary value $M$ on the remaining three sides of $S_{\delta}$. We have that $\left|F_{\delta}^{\bullet}\right| \leq H_{\delta}\left(F_{\delta}^{\bullet}\right.$ is the restriction of $F_{\delta}$ to $\overline{\mathcal{V}}_{\Omega_{\delta}}$ ), by subharmonicity and monotonicity of harmonic 
measure (as $F_{\delta}^{\bullet}$ takes boundary value 0 on $\partial \mathcal{V}_{\Omega_{\delta}}$ ). Hence, by standard harmonic measure estimates (see [DHN11, Lemma 3.5] for instance), we deduce that there exists an absolute constant $C_{1}>0$ such that

$$
\left|F_{\delta}^{\bullet}\left(z+\frac{\delta}{2}\right)\right| \leq \frac{C_{1} M}{\operatorname{dist}\left(z_{\delta},\left\{p_{\delta}^{1}, p_{\delta}^{2}\right\}\right)} \delta .
$$

By again using Proposition 94 and noticing that

$$
\frac{1}{\sqrt{2} \delta}\left|F_{\delta}^{\bullet}\left(z+\frac{\delta}{2}\right)-0\right|=\left|\frac{f_{\delta}^{\mathrm{SPIN}}\left(\Omega_{\delta}, x_{\delta}, \cdot\right)}{\left|f_{\delta}^{\mathrm{SPIN}}\left(\Omega_{\delta}, x_{\delta}, w_{\delta}\right)\right|}\right|^{2},
$$

we obtain that there exists an absolute constant $C_{2}>0$ such that

$$
\left|\frac{f_{\delta}^{\text {SPIN }}\left(\Omega_{\delta}, x_{\delta}, \cdot\right)}{\left|f_{\delta}^{\text {SPIN }}\left(\Omega_{\delta}, x_{\delta}, w_{\delta}\right)\right|}\right| \leq C_{2} \sqrt{\frac{M}{\operatorname{dist}\left(z_{\delta},\left\{p_{\delta}^{1}, p_{\delta}^{2}\right\}\right)}},
$$

which is the desired result.

\section{Appendix A: Assumption on the vertical part of the boundary}

In this subsection, we prove Lemma 7, which is used in Section 4.3 to get our main result.

Lemma (Lemma 7). To prove Theorem 1, we can assume that the domain $D$ is such that the arc $[b, r]$ contains a vertical part $\mathfrak{v}$ and that the discrete domains $D_{\delta}$ are such that the arc $\left[b_{\delta}, r_{\delta}\right]$ contains a vertical part $\mathfrak{v}_{\delta}$ converging to $\mathfrak{v}$ as $\delta \rightarrow 0$.

Proof. This follows from the following monotonicity property of the Ising model: if we

- take $\left(D_{\delta}^{(1)}, r_{\delta}, \ell_{\delta}, b_{\delta}\right)$ and $\left(D_{\delta}^{(2)}, r_{\delta}, \ell_{\delta}, b_{\delta}\right)$ be two discrete domains such that $D_{\delta}^{(1)} \subset D_{\delta}^{(2)}$ such that the domains $D_{\delta}^{(1)}$ and $D_{\delta}^{(2)}$ share the same boundary $\operatorname{arcs}\left[r_{\delta}, \ell_{\delta}\right]$ and $\left[r_{\delta}, b_{\delta}\right]$ (but not necessarily $\left[b_{\delta}, r_{\delta}\right]$ ),

- consider the Ising models on $\left(D_{\delta}^{(1)}, r_{\delta}, \ell_{\delta}, b_{\delta}\right)$ and $\left(D_{\delta}^{(2)}, r_{\delta}, \ell_{\delta}, x_{\delta}\right)$ with dipolar boundary conditions (free on $\left[r_{\delta}, \ell_{\delta}\right]$, - on $\left[\ell_{\delta}, b_{\delta}\right]$ and + on $\left[b_{\delta}, r_{\delta}\right]$ ), at the same inverse temperature,

then we find a coupling $\left(\left(\sigma_{y}^{(1)}\right)_{y \in D_{\delta}^{(1)}},\left(\sigma_{y}^{(2)}\right)_{y \in D_{\delta}^{(2)}}\right)$ of both Ising models in such a way that

$$
\sigma_{y}^{(1)} \geq \sigma_{y}^{(2)} \quad \forall y \in D_{\delta}^{(1)}
$$

and hence that $\gamma_{\delta}^{(1)}$, the dipolar interface on $\left(D_{\delta}^{(1)}, r_{\delta}, \ell_{\delta}, b_{\delta}\right)$, is always to the left of $\gamma_{\delta}^{(2)}$, the dipolar interface on $\left(D_{\delta}^{(2)}, r_{\delta}, \ell_{\delta}, b_{\delta}\right)$.

We can construct a coupling of two Markov chains $\left(\sigma_{y}^{(1)}(t)\right)_{y \in D_{\delta}^{(1)}, t \geq 0},\left(\sigma_{y}^{(2)}(t)\right)_{y \in D_{\delta}^{(2)}, t \geq 0}$ such that $\sigma_{y}^{(1)}(t) \geq \sigma_{y}^{(2)}(t)$ for any $y \in D_{\delta}^{(1)}$ and any time $t \geq 0$, and such that the laws of $\left(\sigma_{y}^{(1)}(t)\right)_{y \in D_{\delta}^{(1)}}$ and $\left(\sigma^{(2)}(t)\right)_{y \in D_{\delta}^{(2)}}$ converge, as $t \rightarrow \infty$, to the Ising model 
probability measures $\left(\sigma_{y}^{(1)}\right)_{y \in D_{\delta}^{(1)}}$ on $D_{\delta}^{(1)}$ and $\left(\sigma_{y}^{(2)}\right)_{y \in D_{\delta}^{(2)}} D_{\delta}^{(2)}$. To construct this Markov chain coupling, we can use Glauber dynamics, starting from a configuration with all spins set to +1 , for instance. $\mathbb{R}$,

From the above coupling, we deduce that for any increasing function $f:\{ \pm 1\}^{D_{\delta}^{(1)}} \rightarrow$

$$
\begin{aligned}
\mathbb{E}\left[f\left(\left(\sigma_{y}^{(1)}\right)_{y \in D_{\delta}^{(1)}}\right)\right] & =\lim _{t \rightarrow \infty} \mathbb{E}\left[f\left(\left(\sigma_{y}^{(1)}(t)\right)_{y \in D_{\delta}^{(1)}}\right)\right] \\
& \geq \lim _{t \rightarrow \infty} \mathbb{E}\left[f\left(\left(\sigma_{y}^{(2)}(t)\right)_{y \in D_{\delta}^{(1)}}\right)\right] \\
& =\mathbb{E}\left[f\left(\left(\sigma_{y}^{(2)}\right)_{y \in D_{\delta}^{(1)}}\right)\right] .
\end{aligned}
$$

In other words, $\left(\sigma_{y}^{(1)}\right)_{y \in D_{\delta}^{(1)}}$ dominates $\left(\sigma_{y}^{(2)}\right)_{y \in D_{\delta}^{(1)}}$ stochastically. By Strassen's theorem [Stra65], this is equivalent to the existence of a coupling satisfying the inequality 13.15 above.

With this monotonicity property, we can now approximate from inside and outside the domain $D$ (and its discretizations) by domains having a vertical part on $[b, r]$, we obtain convergence to dipolar SLE(3) on those domains. The desired result follows readily (the interface can be squeezed between two interfaces whose limit is dipolar SLE(3), and these two interfaces are arbitrarily close to each other.

\section{Appendix B: $\operatorname{SLE}(\kappa ; \rho)$ lemmas}

In this subsection, we prove the characterization of $\operatorname{SLE}(16 / 3 ;-8 / 3)$ provided by Lemma 30. Let us first give the following general characterization of the $\operatorname{SLE}(\kappa ; \rho)$ processes.

Lemma 95. Let $\gamma$ be a chordal $S L E(\kappa)$ in $\mathbb{H}$ from 0 to $\infty$, and $\tilde{\gamma}$ be an $S L E(\kappa ; \rho)$ in $\mathbb{H}$ starting from 0 with force point $x>0$ and observation point $\infty$. For $\epsilon>0$ and $\varrho>0$, let $\vartheta^{\epsilon}$ (respectively $\tilde{\vartheta}^{\epsilon}$ ) be the first time that $\gamma$ (respectively $\tilde{\gamma}$ ) hits

$$
\left\{z:|z| \geq \frac{1}{\epsilon}\right\} \cup\{z: \operatorname{dist}(z,[x, \infty)) \leq \epsilon\} .
$$

Let $\mathcal{P}^{\epsilon}$ be the law of $\gamma\left[0, \vartheta^{\epsilon}\right]$ and $\tilde{\mathcal{P}}^{\epsilon}$ be the law of $\tilde{\gamma}\left[0, \tilde{\vartheta}^{\epsilon}\right]$.

Then $\mathcal{P}^{\epsilon}$ and $\tilde{\mathcal{P}}^{\epsilon}$ are absolutely continuous with respect to each other, and for any $\mu[0, t]$ in their support, we have the follow expression for the Radon-Nikodym derivative:

$$
\frac{\mathrm{d} \tilde{\mathcal{P}}^{\epsilon}}{\mathrm{d} \mathcal{P}^{\epsilon}}(\mu[0, t])=G_{t}^{\prime}(x)^{h}\left(\frac{G_{t}(x)}{x}\right)^{\rho / \kappa}
$$

where $h=\frac{\rho(\rho+4-\kappa)}{4 \kappa}$ and $G_{t}$ is the conformal mapping from (the unbounded component of) $\mathbb{H} \backslash \mu[0, t]$ to $\mathbb{H}$, normalized such that $G_{t}(z) \sim z$ as $z \rightarrow \infty$ and $G_{t}(\mu(t))=0$. 


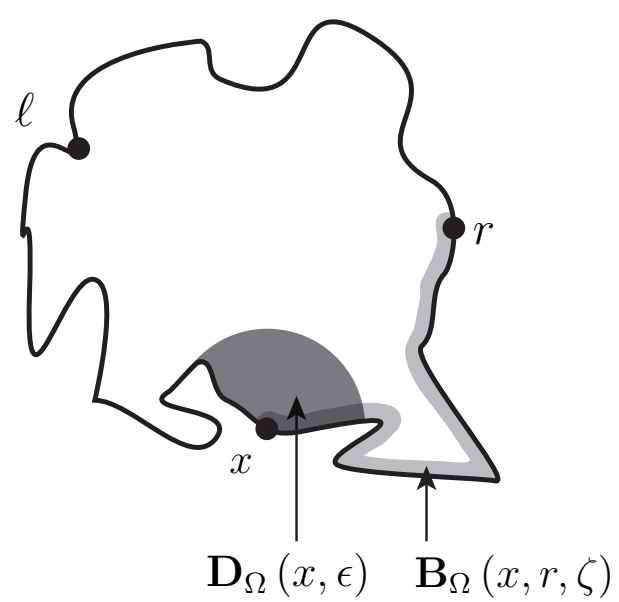

FiguRE 13.8. The domain $(\Omega, r, \ell, x)$ and the neighborhoods $\mathbf{D}_{\Omega}(x, \epsilon)$ and $\mathbf{B}_{\Omega}(x, r, \zeta)$.

Remark 96. When $\kappa=16 / 3$ and $\rho=-8 / 3$, by a straightforward computation, we obtain that the right-hand side of Equation 13.16 can be expressed as

$$
G_{t}^{\prime}(x)^{h}\left(\frac{G_{t}(x)}{x}\right)^{\rho / \kappa}=\frac{\left\langle\sigma_{x}\right\rangle_{H_{t}}^{[-\infty, \mu(t)]_{+}}}{\left\langle\sigma_{x}\right\rangle_{\mathbb{H}}^{[-\infty, 0]_{+}}}
$$

where $H_{t}$ is the unbounded connected component of $\mathbb{H} \backslash \mu[0, t]$.

Proof of Lemma 95. This result is a consequence of Girsanov's theorem. Proofs of similar statements can be found in Wer04, Section 3] or in Kyt06b, Section 1.2.4].

We can now prove Lemma 30 . For definiteness, we take the time parametrization inherited from the time parametrization in the half-plane via the conformal mapping $\varphi:(\mathbb{H}, 0, x, \infty) \rightarrow(\Omega, r, \ell, x)$. Also recall that we denote by $\mathbf{D}_{\Omega}(x, \epsilon)$ the connected component of $\{z \in \bar{\Omega}:|z-x| \leq \epsilon\}$ that contains $x$. Set also $\mathbf{B}_{\Omega}(x, r, \zeta):=$ $\bigcup_{z \in[x, r]} \mathbf{D}_{\Omega}(z, \zeta)$.

Lemma (Lemma 30). Consider the domain $(\Omega, r, \ell, x)$. Let $\lambda$ have the law of an $S L E(16 / 3)$ curve in $\Omega$ from $\ell$ to $r$ and let $\tilde{\lambda}$ have the law of an $S L E(16 / 3 ;-8 / 3)$ curve in $\Omega$ with starting point $\ell$, observation point $r$ and force point $x$.

For $\epsilon>0$, let $\tau^{\epsilon}$ be the (possibly infinite) first time that $\lambda$ hits $\mathbf{D}_{\Omega}(x, \epsilon)$ and let $\tilde{\tau}^{\epsilon}$ be the first time that $\tilde{\lambda}$ hits $\mathbf{D}_{\Omega}(x, \epsilon)$. Let $\mathbb{P}^{\epsilon}$ be the law of $\lambda\left[0, \tau^{\epsilon}\right]$ and $\tilde{\mathbb{P}}^{\epsilon}$ be the law of $\tilde{\lambda}\left[0, \tilde{\tau}^{\epsilon}\right]$. Then we have

$$
\operatorname{Supp}\left(\tilde{\mathbb{P}}^{\epsilon}\right)=\left\{\mu \in \operatorname{Supp}\left(\mathbb{P}^{\epsilon}\right): \mu \text { hits } \mathbf{D}_{\Omega}(x, \epsilon) \text { in finite time and } \mu \cap[x, r]=\emptyset\right\}
$$


and for any curve $\mu[0, t] \in \operatorname{Supp}\left(\tilde{\mathbb{P}}^{\epsilon}\right)$

$$
\frac{\mathrm{d} \tilde{\mathbb{P}}^{\epsilon}}{\mathrm{d} \mathbb{P}^{\epsilon}}(\mu[0, t])=\frac{\left\langle\sigma_{x}\right\rangle_{\Omega \backslash \mu[0, t]}^{[r, \mu(t)]_{+}}}{\left\langle\sigma_{x}\right\rangle_{\Omega}^{[r, \ell]_{+}}} .
$$

Proof. For $\epsilon>0$ and $\zeta>0$, let us denote by $\mathbb{P}^{\epsilon, \zeta}$ (respectively $\tilde{\mathbb{P}}^{\epsilon, \zeta}$ ) the law of $\lambda$ (respectively of $\tilde{\lambda}$ ) stopped as it hits

$$
\mathbf{D}_{\Omega}(x, \epsilon) \cup \mathbf{B}_{\Omega}(\zeta)
$$

It is the enough to show that

- $\mathbb{P}^{\epsilon, \zeta}$ and $\tilde{\mathbb{P}}^{\epsilon, \zeta}$ are absolutely continuous with respect to each other and for any $\mu[0, t]$ in their support,

$$
\frac{\mathrm{d} \tilde{\mathbb{P}}^{\epsilon, \zeta}}{\mathrm{d} \mathbb{P}^{\epsilon, \zeta}}(\mu[0, t])=\frac{\left\langle\sigma_{x}\right\rangle_{\Omega \backslash \mu[0, t]}^{[r, \mu(t)]_{+}}}{\left\langle\sigma_{x}\right\rangle_{\Omega}^{[r, \ell]_{+}}} .
$$

By conformal invariance of SLE and of the right-hand side of this formula, it is enough to show this on the half-plane and the result follows from Lemma 95 and Remark 96 above.

- As $\zeta \rightarrow 0, \tilde{\mathbb{P}}^{\epsilon, \zeta} \rightarrow \tilde{\mathbb{P}}^{\epsilon}$ : in other words, $\tilde{\lambda}\left[0, \tilde{\tau}^{\epsilon}\right]$ almost surely does not hit $[x, r]$. This gives

$$
\operatorname{Supp}\left(\tilde{\mathbb{P}}^{\epsilon}\right) \subset\left\{\mu \in \operatorname{Supp}\left(\mathbb{P}^{\epsilon}\right): \mu \text { hits } \mathbf{D}_{\Omega}(x, \epsilon) \text { and } \mu \cap[x, r]=\emptyset\right\} \text {. }
$$

The inclusion in the other direction immediately follows from Lemma 95.

To conclude the proof it hence remains to show that for any fixed $\epsilon>0$, we have

$$
\tilde{\mathbb{P}}^{\epsilon, \zeta}\left\{\tilde{\lambda}\left[0, \tilde{\tau}^{\epsilon, \zeta}\right] \cap \mathbf{B}_{\Omega}(x, r, \zeta) \neq \emptyset\right\} \underset{\zeta \rightarrow 0}{\longrightarrow} 0
$$

By conformal invariance, it is sufficient to prove this when $\Omega$ is the rectangle

$$
\{z \in \mathbb{C}: \Re \mathfrak{e}(z) \in(-1,1), \Im \mathfrak{m}(z) \in(0,1)\},
$$

with $\ell=-1, x=0$ and $r=1$. For $\mu[0, t] \in \operatorname{Supp}\left(\tilde{\mathbb{P}}^{\epsilon, \zeta}\right)$, by Equation 13.17 and Remark 28, we have

$$
\frac{\mathrm{d} \tilde{\mathbb{P}} \epsilon, \zeta}{\mathrm{d} \mathbb{P} \epsilon, \zeta}(\mu[0, t])=\frac{\left\langle\sigma_{x}\right\rangle_{\Omega \backslash \mu[0, t]}^{[r, \mu(t)]_{+}}}{\left\langle\sigma_{x}\right\rangle_{\Omega}^{[r, \ell]_{+}}}=\text {Cst } \cdot \sqrt{\frac{\partial}{\partial \nu_{\text {int }}(x)} \mathbf{H}_{\Omega \backslash \mu[0, t]}(\cdot,[r, \mu(t)])},
$$

where $\mathbf{H}_{\Omega \backslash \mu[0, t]}(z,[r, \mu(t)])$ is the harmonic measure of $[r, \mu(t)]$ in $\Omega \backslash \mu[0, t]$ viewed from $z$ (see Figure 13.9) and $\frac{\partial}{\partial \nu_{\text {int }}(x)}$ is the inward normal derivative at $x$.

By standard harmonic measure estimates, we have that if $\mu(t) \in \mathbf{B}_{\Omega}(x, r, \zeta)$, then the right-hand side is bounded by Cst $\cdot \zeta^{\alpha}$ for some $\alpha>0$, uniformly with respect to $\mu[0, t]$. From there, we immediately deduce 13.18 . 


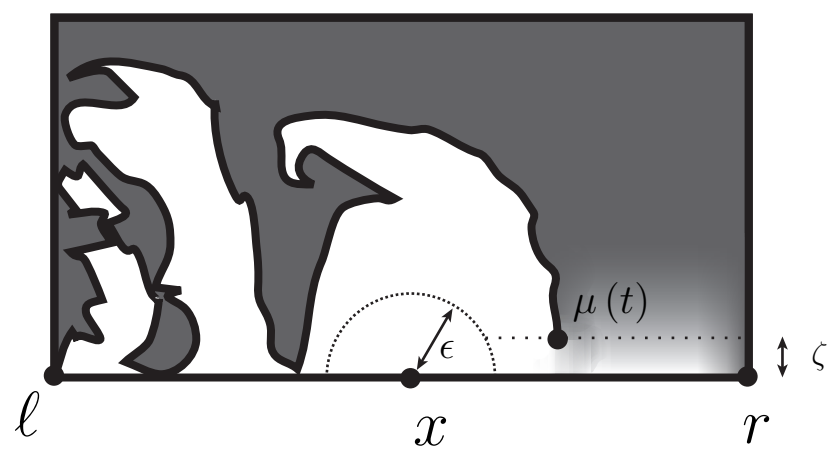

Figure 13.9. Harmonic measure $\mathbf{H}_{\Omega \backslash \mu[0, t]}(\cdot,[r, \mu(t)])$ (dark gray is 1, white is 0 ).

\section{Appendix C: Control of the end of the conditioned interface}

The goal of this subsection is to prove Lemma 33, which is used to conclude the proof of Theorem 32. Recall that $\tilde{\lambda}_{\delta}$ has the law of a critical FK-Ising interface in $\left(\Omega_{\delta}, r_{\delta}, \ell_{\delta}, x_{\delta}\right)$, from $\ell_{\delta}$ to $r_{\delta}$, conditioned to pass at $x_{\delta}$, stopped as it hits $x_{\delta}$ and that $\tilde{\lambda}$ has the law of an $\operatorname{SLE}(16 / 3 ;-8 / 3)$ in $\Omega$ with starting point $\ell$, observation point $r$ and force point $x$.

For $\epsilon>0$, we defined $\mathbf{D}_{\Omega_{\delta}}\left(x_{\delta}, \epsilon\right)$ as the connected component of $\left\{z \in \Omega_{\delta}:|z-x| \leq \epsilon\right\}$ that contains $x_{\delta}$ and $\mathbf{D}_{\Omega}(x, \epsilon)$ as the connected component of $\{z \in \bar{\Omega}:|z-x| \leq \epsilon\}$ containing $x$. For $\rho>\epsilon>0$ let us also define $\mathbf{A}_{\Omega_{\delta}}\left(x_{\delta}, \epsilon, \rho\right)$ as $\mathbf{D}_{\Omega_{\delta}}\left(x_{\delta}, \rho\right) \backslash \mathbf{D}_{\Omega_{\delta}}\left(x_{\delta}, \epsilon\right)$.

Lemma (Lemma 33 ). For any $\rho>0$, the probability that $\tilde{\lambda}_{\delta}$ exits $\mathbf{D}_{\Omega_{\delta}}\left(x_{\delta}, \rho\right)$ after the time $\tilde{\tau}_{\delta}^{\epsilon}$ tends to 0 as $\epsilon \rightarrow 0$, uniformly with respect to $\left(\Omega_{\delta}, r_{\delta}, \ell_{\delta}, x_{\delta}\right)$. Similarly, for any $\rho>0$, the probability that $\tilde{\lambda}$ exits $\mathbf{D}_{\Omega}(x, \rho)$ after time $\tilde{\tau}^{\epsilon}$ tends to 0 as $\epsilon \rightarrow 0$, uniformly with respect to $(\Omega, r, \ell, x)$.

To prove these lemmas, we will use the following property of FK interfaces conditioned to pass at a boundary point (see Figure 13.10).

Lemma 97. Let $\left(\Omega_{\delta}, r_{\delta}, \ell_{\delta}, x_{\delta}\right)$ be a discrete domain and let $\lambda_{\delta}^{*}$ have the law of an $F K$ interface from $\ell_{\delta}$ to $r_{\delta}$, conditioned to pass at $x_{\delta}$. Let $\lambda_{\delta}^{\ell}$ denote the part of $\lambda_{\delta}^{*}$ from $\ell_{\delta}$ to $x_{\delta}$ and let $\lambda_{\delta}^{r}$ denote the part of $\lambda_{\delta}^{*}$ from $x_{\delta}$ to $r_{\delta}$. Then, conditionally on $\lambda_{\delta}^{r}, \lambda_{\delta}^{\ell}$ has the law of an (unconditioned) $F K$ interface in $\left(\Omega_{\delta}^{\ell}, \ell_{\delta}, x_{\delta}\right)$ from $\ell_{\delta}$ to $x_{\delta}$, where $\Omega_{\delta}^{\ell}$ is the connected component of $\Omega_{\delta} \backslash \lambda_{\delta}^{r}$ containing $\ell_{\delta}$. The law of $\lambda_{\delta}^{r}$ conditionally on $\lambda_{\delta}^{\ell}$ is described symmetrically (echanging $r$ and $\ell$ ).

Proof. This is a direct consequence of the domain Markov property of the FK model.

We will also use the following uniform lemma concerning the behavior of (unconditioned) FK interface near their endpoints:

Lemma 98. Let $\left(\Omega_{\delta}, r_{\delta}, \ell_{\delta}\right)$ be a discrete domain and let $\lambda_{\delta}$ have the law of a critical FK-Ising interface from $\ell_{\delta}$ to $r_{\delta}$. Then for any $\rho>0$, the probability that $\lambda_{\delta}$ 

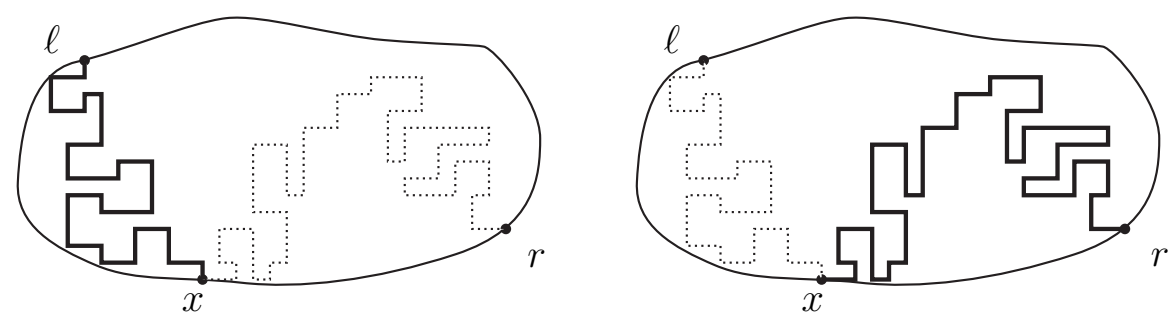

FiguRE 13.10. Law of $\lambda_{\delta}^{\ell}$ knowing $\lambda_{\delta}^{r}$ and conversely.

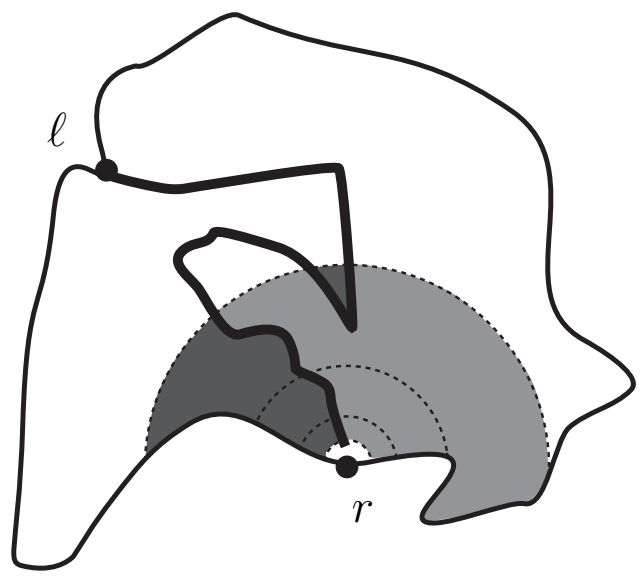

FiguRE 13.11. Nested family of annuli near the target point.

exits $\mathbf{D}_{\Omega_{\delta}}\left(r_{\delta}, \rho\right)$ after entering $\mathbf{D}_{\Omega_{\delta}}\left(r_{\delta}, \epsilon\right)$ is bounded by $C(\epsilon / \rho)^{\vartheta}$, for some universal constants $C, \vartheta>0$.

Proof. The result follows from standard techniques, using RSW crossing type estimates, as explained in [KeSm11a, Section 3.3]. The RSW estimates are given by [ChSm09] or [DHN11. The idea is to show that once $\lambda$ has crossed an annulus, with uniformly positive probability, one can guarantee that $\lambda$ will not cross this annulus anymore, and to do this for a family of concentric annuli (see Figure 13.11).

The third ingredient we will need is the following monotonicity lemma:

Lemma 99. Let $\left(\Omega_{\delta}^{(1)}, r_{\delta}, \ell_{\delta}\right)$ and $\left(\Omega_{\delta}^{(2)}, r_{\delta}, \ell_{\delta}\right)$ be two discrete domains such that $\Omega_{\delta}^{(2)} \subset \Omega_{\delta}^{(1)}$ and such that the arcs $\left[\ell_{\delta}, r_{\delta}\right]$ in $\Omega_{\delta}^{(1)}$ and in $\Omega_{\delta}^{(2)}$ are the same. Consider the FK-Ising interfaces $\lambda_{\delta}^{(1)}$ in $\left(\Omega_{\delta}^{(1)}, r_{\delta}, \ell_{\delta}\right)$ and $\lambda_{\delta}^{(2)}$ in $\left(\Omega_{\delta}^{(2)}, r_{\delta}, \ell_{\delta}\right)$, both oriented from $\ell_{\delta}$ to $r_{\delta}$. Then there is a coupling of $\lambda_{\delta}^{(1)}$ and $\lambda_{\delta}^{(2)}$ such that $\lambda_{\delta}^{(2)}$ is always to the right of $\lambda_{\delta}^{(1)}$ (i.e. $\lambda_{\delta}^{(2)}$ separates $\lambda_{\delta}^{(1)}$ from $\left[\ell_{\delta}, r_{\delta}\right]$ in $\left.\Omega_{\delta}^{(1)}\right)$.

Proof. This follows from the strong positive association property of the FK model (see [Gri06]). 
Using the three lemmas we can now prove the desired result:

Proof of Lemma 33. Let us first prove the discrete statement. Denote by $\lambda_{\delta}^{*}$ the full interface from $\ell_{\delta}$ to $r_{\delta}$, conditioned to pass at $x_{\delta}$; denote by $\lambda_{\delta}^{\ell}$ the part of $\lambda_{\delta}^{*}$ form $\ell_{\delta}$ to $x_{\delta}$ (hence $\lambda_{\delta}^{\ell}$ is the same as $\tilde{\lambda}_{\delta}$ in the statement) and by $\lambda_{\delta}^{r}$ the part of $\lambda_{\delta}^{*}$ from $x_{\delta}$ to $r_{\delta}$.

If $\lambda_{\delta}^{\ell}$ exits $\mathbf{D}_{\Omega_{\delta}}\left(x_{\delta}, \rho\right)$ after the time $\tilde{\tau}_{\delta}^{\epsilon}$, it either exits on the left or on the right of $\lambda_{\delta}^{\ell}\left[0, \tilde{\tau}_{\delta}^{\epsilon}\right]$, i.e. in the connected component of $\mathbf{A}_{\Omega_{\delta}}\left(x_{\delta}, \epsilon, \rho\right) \backslash \lambda_{\delta}^{\ell}\left[0, \tilde{\tau}_{\delta}^{\epsilon}\right]$ that is bounded by the left side (respectively the right side) of $\lambda_{\delta}^{\ell}$ (see Figures 13.12 and 13.13). Let us decompose along these two cases:

- The probability of the event $\mathfrak{L}$ that $\lambda_{\delta}^{\ell}$ exits $\mathbf{D}_{\Omega_{\delta}}\left(x_{\delta}, \rho\right)$ on the left of $\lambda_{\delta}^{\ell}\left[0, \tilde{\tau}_{\delta}^{\epsilon}\right]$ can be bounded as follows (see Figure 13.12).

- If we condition on (the whole path) $\lambda_{\delta}^{r}$ and on $\lambda_{\delta}\left[0, \tilde{\tau}_{\delta}^{\epsilon}\right]$, then by Lemma 97, the remaining part of $\lambda_{\delta}^{\ell}$ has the same law as an unconditioned FK interface $\lambda_{\delta}^{(2)}$ in $\left(\Omega_{\delta}^{\dagger}, \lambda_{\delta}\left(\tilde{\tau}_{\delta}^{\epsilon}\right), x_{\delta}\right)$ from $\lambda_{\delta}\left(\tilde{\tau}_{\delta}^{\epsilon}\right)$ to $x_{\delta}$, where $\Omega_{\delta}^{\dagger}$ is the connected component of $\Omega_{\delta} \backslash\left(\lambda_{\delta}\left[0, \tilde{\tau}_{\delta}^{\epsilon}\right] \cup \lambda_{\delta}^{r}\right)$ containing $\lambda_{\delta}\left(\tilde{\tau}_{\delta}^{\epsilon}\right)$.

- By Lemma 99, the interface $\lambda_{\delta}^{(2)}$ can be coupled to always be on the right of an unconditioned FK interface $\lambda_{\delta}^{(1)}$ in $\left(\Omega_{\delta} \backslash \lambda_{\delta}\left[0, \tilde{\tau}_{\delta}^{\epsilon}\right], \lambda_{\delta}\left(\tilde{\tau}_{\delta}^{\epsilon}\right), x_{\delta}\right)$, oriented from $\lambda_{\delta}\left(\tilde{\tau}_{\delta}^{\epsilon}\right)$ to $x_{\delta}$. Hence, the probability that $\lambda_{\delta}^{(2)}$ exits $\mathbf{D}_{\Omega_{\delta}}\left(x_{\delta}, \rho\right)$ on the left of $\lambda_{\delta}^{\ell}\left[0, \tilde{\tau}_{\delta}^{\epsilon}\right]$ is smaller than the probability that $\lambda_{\delta}^{(1)}$ exits $\mathbf{D}_{\Omega_{\delta}}\left(x_{\delta}, \rho\right)$ (through the coupling, the first event implies the second one).

- By Lemma 98 the probability that $\lambda_{\delta}^{(1)}$ exits $\mathbf{D}_{\Omega_{\delta}}\left(x_{\delta}, \rho\right)$ is bounded by $C(\epsilon / \rho)^{\vartheta}$, where $C$ and $\vartheta$ are universal positive constants.

- The probability of the event $\mathfrak{R}$ that $\lambda_{\delta}^{\ell}$ exits $\mathbf{D}_{\Omega_{\delta}}\left(x_{\delta}, \rho\right)$ on the right of $\lambda_{\delta}^{\ell}\left[0, \tilde{\tau}_{\delta}^{\epsilon}\right]$ can be bounded in the following way (see Figure 13.13.

- For $\phi>\epsilon$, let $E_{\delta}^{\phi}$ be the event that $\lambda_{\delta}^{r}$ exits $\mathbf{D}_{\Omega_{\delta}}\left(x_{\delta}, \phi\right)$ to the right of $\lambda_{\delta}^{r}$ after entering $\mathbf{D}_{\Omega_{\delta}}\left(x_{\delta}, \epsilon\right)$ (we parametrize $\lambda_{\delta}^{r}$ from $r$ to $x$ ). By the bound of the previous paragraph, applied to $\lambda_{\delta}^{r}$, the probability of $E_{\delta}^{\phi}$ is bounded by $C\left(\frac{\phi}{\rho}\right)^{\vartheta}$.

- By Lemma 97, for $0<\epsilon<\phi<\rho$, conditionally on $\lambda_{\delta}^{r}$ and on the event that $E^{\phi}$ does not occur, the probability that $\lambda_{\delta}^{\ell}$ exits $\mathbf{D}_{\Omega_{\delta}}\left(x_{\delta}, \rho\right)$ after time $\tilde{\tau}_{\delta}^{\epsilon}$ is bounded by the probability that an unconditioned FK interface (in $\Omega_{\delta}^{\ell}$, from $\ell_{\delta}$ to $x_{\delta}$ ) exits $\mathbf{D}_{\Omega_{\delta}^{\ell}}\left(x_{\delta}, \rho\right)$ after entering $\mathbf{D}_{\Omega_{\delta}^{r}}\left(x_{\delta}, 2 \phi\right)$. By Lemma 98 , this probability is bounded by $C\left(\frac{2 \phi}{\rho}\right)^{\vartheta}$. 
- Writing $\mathrm{E}_{k}$ for $E_{\delta}^{2^{k} \epsilon}$, and summing over successive scales, we get:

$$
\begin{aligned}
\mathbb{P}(\mathfrak{R}) & =\sum_{k=0}^{\left\lfloor\log _{2}(\rho / \epsilon)\right\rfloor} \mathbb{P}\left\{\mathfrak{R} \mid \mathrm{E}_{k} \backslash \mathrm{E}_{k+1}\right\} \mathbb{P}\left\{\mathrm{E}_{k} \backslash \mathrm{E}_{k+1}\right\} \\
& \leq \sum_{k=0}^{\left\lfloor\log _{2}(\rho / \epsilon)\right\rfloor} \mathbb{P}\left\{\mathfrak{R} \mid \mathrm{E}_{k} \backslash \mathrm{E}_{k+1}\right\} \mathbb{P}\left\{\mathrm{E}_{k}\right\} \\
& \leq \sum_{k=0}^{\left\lfloor\log _{2}(\rho / \epsilon)\right\rfloor} C^{2}\left(\frac{2^{k-1} \epsilon}{\rho}\right)^{\vartheta}\left(\frac{\epsilon}{2^{k} \epsilon}\right)^{\vartheta} \\
& \leq \tilde{C}\left(\frac{\epsilon}{\rho}\right)^{\vartheta}\left(1+\log \left(\frac{\rho}{\epsilon}\right)\right)
\end{aligned}
$$

Given the two uniform bounds for the probabilities of $\mathfrak{L}$ and $\mathfrak{R}$ above, we obtain the desired result for the conditioned FK interface $\tilde{\lambda}_{\delta}$.

For the $\operatorname{SLE}(16 / 3 ;-8 / 3)$ curve $\tilde{\lambda}$, we get the same uniform bound as for the FK interface. For $\epsilon, \rho>0$, let us denote by $\mathbf{T}_{\delta}(\epsilon, \rho)$ (respectively $\mathbf{T}(\epsilon, \rho)$ ) the possibly infinite first time when $\tilde{\lambda}_{\delta}$ (respectively $\tilde{\lambda}$ ) exits $\mathbf{D}_{\Omega_{\delta}}\left(x_{\delta}, \rho\right)$ (respectively $\mathbf{D}_{\Omega}(x, \rho)$ ) after time $\tilde{\tau}_{\delta}^{\epsilon}$ (respectively $\tilde{\tau}^{\epsilon}$ ). We have

$$
\begin{aligned}
\mathbb{P}\left\{\tilde{\tau}^{\epsilon}<\mathbf{T}(\epsilon, \rho)<\infty\right\} & =\mathbb{P}\left(\bigcup_{\alpha \in(0, \epsilon)}\left\{\tilde{\tau}^{\epsilon}<\mathbf{T}(\epsilon, \rho)<\tilde{\tau}^{\alpha}\right\}\right) \\
& =\sup _{\alpha \in(0, \epsilon)} \mathbb{P}\left\{\tilde{\tau}^{\epsilon}<\mathbf{T}(\epsilon, \rho)<\tilde{\tau}^{\alpha}\right\} .
\end{aligned}
$$

But for any $\alpha \in(0, \epsilon)$, as $\tilde{\lambda}\left[0, \tilde{\tau}^{\alpha}\right]$ is the scaling limit of $\tilde{\lambda}_{\delta}\left[0, \tilde{\tau}_{\delta}^{\alpha}\right]$, we have

$$
\begin{aligned}
\mathbb{P}\left\{\tilde{\tau}^{\epsilon}<\mathbf{T}(\epsilon, \rho)<\tilde{\tau}^{\alpha}\right\} & \leq \limsup _{\delta \rightarrow 0} \mathbb{P}\left\{\tilde{\tau}_{\delta}^{\epsilon}<\mathbf{T}_{\delta}(\epsilon, \rho)<\tilde{\tau}_{\delta}^{\alpha}\right\} \\
& \leq \limsup _{\delta \rightarrow 0} \mathbb{P}\left\{\tilde{\tau}_{\delta}^{\epsilon}<\mathbf{T}_{\delta}(\epsilon, \rho)\right\}
\end{aligned}
$$

But $\mathbb{P}\left\{\tilde{\tau}_{\delta}^{\epsilon}<\mathbf{T}_{\delta}(\epsilon, \rho)\right\} \rightarrow 0$ uniformly as $\epsilon \rightarrow 0$ by the first part of the lemma, so we obtain the desired result.

\section{References}

[AbSt64] M. Abramowitz and I. A. Stegun: Handbook of mathematical functions, Dover Publications Inc., 1964.

[AiBu99] M. Aizenman and A. Burchard, Hölder regularity and dimension bounds for random curves. Duke Math. J. 99:419-453, 1999.

[Bax89] R. Baxter, Exactly solved models in statistical mechanics. Academic Press Inc. [Harcourt Brace Jovanovich Publishers], London, 1989.

[BBH05] M. Bauer, D. Bernard, J. Houdayer, Dipolar stochastic Loewner evolutions, J. Stat. Mech. (3), P03001, 18pp. (electronic), 2005.

[BPZ84a] A. A. Belavin, A. M. Polyakov, A. B. Zamolodchikov. Infinite conformal symmetry in two-dimensional quantum field theory. Nucl. Phys. B, 241(2):333-380, 1984.

[BPZ84b] A. A. Belavin, A. M. Polyakov, A. B. Zamolodchikov. Infinite conformal symmetry of critical fluctuations in two dimensions. J. Stat. Phys., 34(5-6):763-774, 1984. 


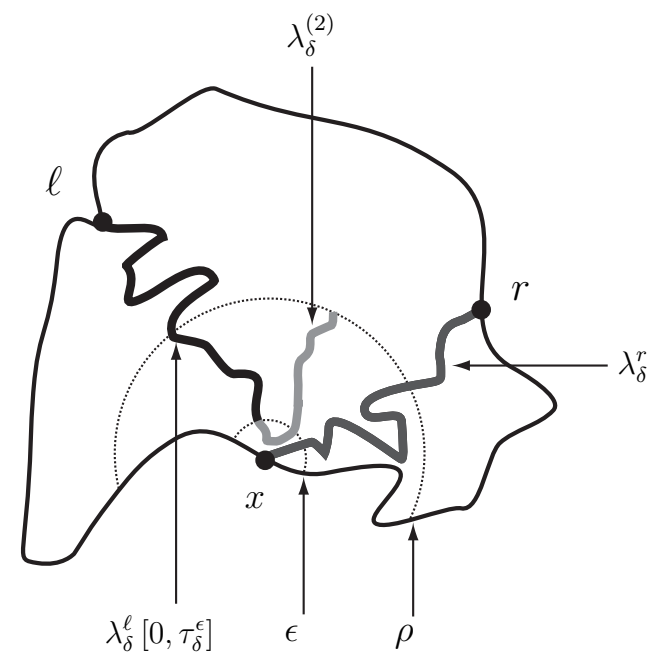

FiguRE 13.12. Event $\mathfrak{L}:$ exit on the left of $\lambda_{\delta}^{\ell}\left[0, \tilde{\tau}_{\delta}^{\epsilon}\right]$

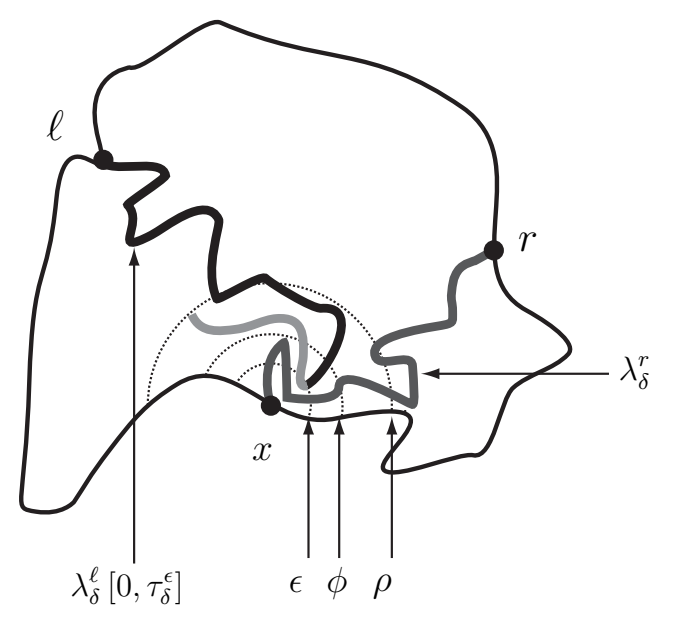

Figure 13.13. Event $\mathfrak{R}$ : exit on the right of $\lambda_{\delta}^{\ell}\left[0, \tilde{\tau}_{\delta}^{\epsilon}\right]$

[BeIz10] D. Beliaev, K. Izyurov, Proof of a factorization formula for critical percolation. arXiv:1011.5822.

[BeDu11] S. Benoist, J. Dubédat, in preparation.

[CaNe07a] F. Camia and C.M. Newman, Critical percolation exploration path and SLE(6): a proof of convergence. Probab. Th. Rel. Fields 139:473-519, 2007.

[CaNe07b] F. Camia and C. M. Newman, Two-Dimensional Critical Percolation: The Full Scaling Limit. Comm. Math. Phys. 268(1):1-38, 2007.

[Car84] J. Cardy, Conformal invariance and Surface Critical Behavior. Nucl. Phys. B 240:514$532,1984$.

[Car92] J. Cardy. Critical percolation in finite geometries. J. Phys. A 25(4):L201-L206, 1992.

[ChSm11] D. Chelkak and S. Smirnov, Discrete complex analysis on isoradial graphs. Advances in Mathematics, 228:1590-1630, 2011.

[ChSm09] D. Chelkak and S. Smirnov, Universality in the 2D Ising model and conformal invariance of fermionic observables. Inventiones Math., to appear. arXiv:0910.2045.

[ChSm11] D. Chelkak and S. Smirnov, preprint. 
[Che11] D. Chelkak, in preparation.

[CDH11] D. Chelkak, H. Duminil-Copin, C. Hongler, in preparation.

[DMS97] P. Di Francesco, P. Mathieu, D. Sénéchal, Conformal Field Theory. Graduate texts in contemporary physics. Springer-Verlag New York, 1997.

[DHN11] H. Duminil-Copin, C. Hongler, P. Nolin, Connection probabilities and RSW-type bounds for the FK Ising model. Comm. Pure. Appl. Math. 64(9):1165-1198, 2011.

[Fis98] M. E. Fisher, Renormalization group theory: its basis and formulation in statistical mechanics. Rev. Modern Phys., 70(2):653-681, 1998.

[GRS08] C. Garban, S. Rohde, O. Schramm, Continuity of the SLE trace in simply connected domains. Israel Journal of Math., to appear, 2008.

[Gri06] G. Grimmett, The Random-Cluster Model. Volume 333 of Grundlehren der Mathematischen Wissenschaften, Springer-Verlag, Berlin, 2006.

[Hon10a] C. Hongler, Conformal invariance of Ising model correlations. Ph.D. thesis, University of Geneva, http://www.math.columbia.edu/ hongler/thesis.pdf, 2010.

[HoSm10b] C. Hongler, S. Smirnov. The energy density in the critical planar Ising model. arXiv:1008.2645.

[Isi25] E. Ising, Beitrag zur Theorie des Ferromagnetismus. Zeitschrift für Physik, 31:253258,1925 .

[KaCe71] L. Kadanoff, H. Ceva, Determination of an operator algebra for the two-dimensional Ising model. Phys. Rev. B (3), 3:3918-3939, 1971.

[Kem10] A. Kemppainen, Stationarity of SLE. J. Stat. Phys, 139:108-121, 2010.

[KeSm11a] A. Kemppainen, S. Smirnov, preprint. http://wiki.helsinki.fi/display/mathphys/kemppainen

[KeSm11b] A. Kemppainen, S. Smirnov, in preparation.

[Ken00] R. Kenyon, Conformal invariance of domino tiling. Ann. Probab., 28:759-795, 2000.

[Kyt06a] K. Kytölä, On conformal field theory of $\operatorname{SLE}(\kappa ; \rho)$, Journal of Statistical Physics, 123(6):1169-1181, 2006.

[Kyt06b] K. Kytölä, Ph.D, thesis, 2006.

[KrWa41] H. A. Kramers and G. H. Wannnier, Statistics of the two-dimensional ferromagnet. I. Phys. Rev. (2), 60:252-262, 1941.

[LLS00] R. Langlands, M. A. Lewis, Y. Saint-Aubin, Universality and conformal invariance for the Ising model in domains with boundary, J. Stat. Phy. 98(1-2):131-244, 2000.

[LPS94] R. Langlands, P. Pouliot, Y. Saint-Aubin, Conformal invariance in two-dimensional percolation. Bull. Amer. Math. Soc. (N.S.), 30(1):1-61, 1994.

[Law05] G. F. Lawler, Conformally Invariant Processes in the Plane. Americ. Math. Soc, 2005.

[LSW01] G. F. Lawler, O. Schramm, W. Werner, Values of Brownian intersection exponents I: Half-plane exponents. Acta Mathematica. 187:275-308, 2001.

[LSW04] G. F. Lawler, O. Schramm, W. Werner, Conformal invariance of planar loop-erased random walks and uniform spanning tree. Ann. of Probab. 32:939-994, 2004.

[Len20] W. Lenz, Beitrag zum Verständnis der magnetischen Eigenschaften in festen Körpern. Phys. Zeitschr., 21:613-615, 1920.

[MaSm09] N. Makarov and S. Smirnov, Off-critical lattice models and SLE. In Exner, Pavel (ed.), XVIth International Congress on Mathematical Physics, Prague. 362-371, 2009.

[McWu73] B. M. McCoy and T. T. Wu, The two-dimensional Ising model. Harvard University Press, Cambridge, Massachusetts, 1973.

[Mil10] J. Miller, Universality for SLE(4). arXiv:1010.1356v1.

[Ons44] L. Onsager, Crystal statistics. I. A two-dimensional model with an order-disorder transition. Phys. Rev. 65(2):117-149, 1944.

[Pal07] J. Palmer, Planar Ising correlations. Birkhäuser, 2007.

[Pei36] R. Peierls, On Ising's model of ferromagnetism. Proc. Camb. Philos. Soc., 32:477-481, 1936.

[PfVe99] C.-E. Pfister, Y. Velenik, Interface, surface tension and reentrant pinning 2D transition in the 2D Ising model, Comm. Math. Phys. 204(2):269-312, 1999.

[Pom92] C. Pommerenke, Boundary Behavior of Conformal Maps, A Series of Comprehensive Studies in Mathematics 299. Springer-Verlag, Berlin, 1992.

[RoSc05] S. Rohde, O. Schramm, Basic properties of SLE. Annals of Math. 161:883-924, 2005. 
[Sch00] O. Schramm, Scaling limits of loop-erased random walks and uniform spanning trees. Israel J. Math., 118:221-288, 2000.

[Sch07] O. Schramm. Conformally invariant scaling limits: an overview and a collection of problems. International Congress of Mathematicians Vol. I, 513-54, Eur. Math. Soc., Zürich, 2007.

[ScSh09] O. Schramm and S. Sheffield. Contour lines of the two-dimensional discrete Gaussian free field. Acta Math., 202(1):21-137, 2009.

[ScWi05] O. Schramm, D. B. Wilson, SLE coordinate changes, New York J. Math, 11:659-669, 2005.

[She09] S. Sheffield, Exploration trees and conformal loop ensembles. Duke Math. J. 147(1):79-129, 2009.

[ShWe10a] S. Sheffield, W. Werner, Conformal Loop Ensembles: Construction via Loop-soups, arXiv:1006.2373.

[ShWe10b] S. Sheffield, W. Werner, Conformal Loop Ensembles: The Markovian Characterization, arXiv:1006.2374.

[Smi01] S. Smirnov, Critical percolation in the plane: conformal invariance. Cardy's formula, scaling limits, C. R. Acad. Sci. Paris Sér. I Math., 333, 3:239-244, 2001.

[Smi09] S. Smirnov, Critical percolation in the plane. Preprint. arXiv:0909.4499.

[Smi06] S. Smirnov, Towards conformal invariance of 2D lattice models. Sanz-Solé, Marta (ed.) et al., Proceedings of the international congress of mathematicians (ICM), Madrid, Spain, August 22-30, 2006. Volume II: Invited lectures, 1421-1451. Zürich: European Mathematical Society (EMS), 2006.

[Smi10a] S. Smirnov, Conformal invariance in random cluster models. I. Holomorphic fermions in the Ising model. Annals of Math. 172(2):1435-1467, 2010.

[Smi10b] S. Smirnov, Discrete complex analysis and probability. Proceedings of the ICM, Hyderabad, India, to appear, 2010.

[Stra65] , The existence of probability measures with given marginals. The Annals of Mathematical Statistics, 36:423-439, 1965.

[Wer04] W. Werner, Girsanov's transformation for $\operatorname{SLE}(\kappa, \rho)$ processes, intersection exponents and hiding exponents. Ann. Fac. Sci. Toulouse, 13:121-148, 2004.

[Wer10] W. Werner, Percolation et modèle d'Ising. Cours Spécialisés de la SMJ 16, 2010.

[Zha04] D. Zhan, Random Loewner chains in Riemann surfaces. PhD dissertation, Caltech, 2004.

Department of Mathematics, Columbia University. 2990 Broadway, New York, NY 10027, USA.

E-mail address: hongler@math.columbia.edu

Department of Mathematics and Statistics, P. O. Box 68, Fin-00014 University OF HELSinki, Finland

E-mail address: kalle.kytola@helsinki.fi 